\title{
Conformal Properties of Generalized

\author{
Dirac Operator
}

\section{Dissertation}

zur Erlangung des mathematisch-naturwissenschaftlichen

Doktorgrades

"Doctor rerum naturalium"

an der Georg-August-Universität Göttingen

vorgelegt von

\section{Varun Thakre}

aus Nagpur(Staat Maharashtra), Indien.

Göttingen, 2013 


\section{Mitglieder des Betreuungsausschusses:}

Referentin/Referent: Prof. Dr. Victor Pidstrygach

Koreferentin/Koreferent: Prof. Dr. Thomas Schick

\section{Mitglieder des Betreuungsausschusses:}

Prof. Dr. Max Wardetzky (Institut für Num. und Angew. Mathematik)

PD Dr. Katharina Habermann (SUB, George-August-Universität)

Prof. Dr. Laura Covi (Institut für Theoritische Physik)

Prof. Dr. Dominic Schuhmacher (Institut für Mathematische Stochastik)

Tag der mündlichen Prüfung: 5 June 2013 


\begin{abstract}
In this thesis we study the non-linear Dirac operator in dimension four and the associated generalization of the Seiberg-Witten equations in dimension four. The central object of this generalization is a hyperKähler manifold $M$, admitting certain symmetries. The non-linear Dirac operator acts on generalized spinors, which are equivariant maps taking values in $M$. Restricting to a special case of Swann bundles allows us to study the behaviour of the non-linear Dirac operator under the conformal change of metrics on the base manifold.

Harmonic spinors are generalizations of aholomorphic maps between hyperKähler manifolds. The Weitzenböck formula for the non-linear Dirac operator can be interpreted as an energy identity for generalized spinors, analogous to the one satisfied by maps between hyperKähler manifolds. In the light of this comparison, we analyze the behaviour of the energies under smooth deformations of the base manifold. This is the first step in deriving a blow-up condition for harmonic spinors with bounded energies, as in the case of aholomorphic maps.

In the final part, we prove that restricted to the case when the target hyperKähler manifold is a hyperKähler reduction of a flat-space, a harmonic spinor is $L^{\infty}$ and hence also uniformly $W^{1,2}$-bounded. We conclude with some remarks towards understanding the singular set of harmonic spinors.
\end{abstract}




\section{Acknowledgements}

It is my pleasure to thank everyone who in one way or the other have contributed and given their valuable assistance in completion of this dissertation.

First and foremost I extend my sincere thanks and gratitude to my advisor Prof. Victor Pidstrygach for not only introducing me to the world of Gauge Theory but also helping me broaden my horizons beyond my main research through his constant support, encouragement, endless enthusiasm and long discussions during the "tea seminar". Given that I picked up Differential Geometry from scratch after joining his group, he has guided me through things with a lot of patience, attention \& care. I would like to thank Prof. Thomas Schick for his commitment as my co-advisor. I also take this opportunity to thank Prof. Pablo Ramacher for giving me the opportunity to work at Mathematisches Institut.

I thank DFG for supporting me financially for the first three years and the Graduiertenkolleg for supporting me financially thereafter.

Special thanks to my colleague and friend Martin Callies for a taking time out to proof read the entire manuscript and painstakingly looking into every detail of the same. I also thank him for range of interesting discussions on mathematics, photography and politics and his help with even the silliest of my questions.

A word of thanks to all my friends in Göttingen for being my family here away from home and making my stay in Göttingen very pleasant and memorable. They have been a constant source of inspiration. I would also like thank Aprameyan Parthasarthy for all the help with my stay in the initial few months of my joining in Göttingen.

Last, but not the least, I thank my parents for their unwaivering support, encouragement and giving me the freedom pursue my research interests in difficult times. Without it all, this would have remained a remote dream. 


\section{Contents}

1 Introduction 1

2 Preliminaries and notations 5

2.1 Fibre bundles . . . . . . . . . . . . . . . . . . 5

2.2 Principal bundles . . . . . . . . . . . . . . . . . 6

2.2.1 Connections on principal bundles . . . . . . . . . 7

2.2.2 Connections on Associated bundles . . . . . . . . . . . 7

2.3 Vector Bundle . . . . . . . . . . . . . . . . . . . . . . . . . 8

2.4 Action of the gauge group . . . . . . . . . . . . . . 8

2.4 .1 Gauge Group . . . . . . . . . . . . . . . . 8

2.5 Elements of Spin Geometry . . . . . . . . . . . . . . . . . 10

2.5.1 Clifford algebras and Spin Groups . . . . . . . . . . . . . 10

2.5.2 Clifford modules and Spin representations . . . . . . . . . 13

2.5.3 Clifford multiplication . . . . . . . . . . . . . . . 16

2.5.4 Spin structures . . . . . . . . . . . . . . . . . . . 16

2.5.5 Spinor bundles and Dirac operators . . . . . . . . . . . . . 17

2.5.6 $\operatorname{Spin}^{c}(n)$-structures . . . . . . . . . . . . . . 18

3 HyperKähler manifolds $\quad 20$

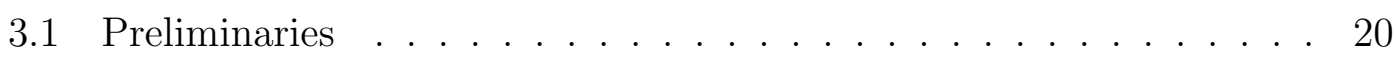

3.1.1 HyperKähler Manifolds . . . . . . . . . . . . . . . . . . . . . 21

3.2 Properties of hyperKähler manifolds with permuting action . . . . . 22

3.2.1 Permuting action . . . . . . . . . . . . . . . . 22

3.2.2 HyperKähler potential . . . . . . . . . . . . . . . . 26

3.2.3 HyperKähler Moment map . . . . . . . . . . . . . . . . . . . 28 
3.3 Quaternionic Vector Spaces . . . . . . . . . . . . . . . . . . 29

3.4 Swann Bundles over Wolf Spaces. . . . . . . . . . . . . . . . . . . . 32

3.4.1 Swann Bundles . . . . . . . . . . . . . . . . . . . . 33

3.4 .2 Orbit Spaces . . . . . . . . . . . . . . 33

4 Generalized Dirac Operator $\quad 35$

$4.1 \operatorname{Spin}_{\varepsilon}^{G}(n)$-structure . . . . . . . . . . . . . . . . 35

4.1 .1 The group $\operatorname{Spin}_{\varepsilon}^{G}(n) \ldots \ldots \ldots \ldots$. . . . . . . . . . . . . . . . . . . . . . . 35

$4.1 .2 \operatorname{Spin}_{\varepsilon}^{G}(n)$ structure . . . . . . . . . . . . . 36

4.2 Generalized Spinors . . . . . . . . . . . . . . . . . 37

4.3 Non-linear Dirac Operator . . . . . . . . . . . . . . . . . 38

4.3.1 Clifford Multiplication . . . . . . . . . . . . . 38

4.3.2 The Covariant derivative . . . . . . . . . . . . . . . . . . 39

4.3.3 The Dirac Operator . . . . . . . . . . . . . . . . . . . . 40

4.4 Conformal property of the non-linear Dirac operator . . . . . . . . . 40

4.4.1 The $C \operatorname{Spin}_{\varepsilon}^{G}(4)$ structure . . . . . . . . . . . . . . . 41

4.4.2 Generalized Dirac operator \& Conformal change of metric . 42

4.5 Weitzenböck Formula . . . . . . . . . . . . . . . . . . . 47

4.6 Fueter operator and the generalized Dirac operator . . . . . . . . . 48

4.6.1 Algebraic Preliminaries . . . . . . . . . . . . . . . . . 48

4.7 A brief survey on Aholomorphic maps . . . . . . . . . . . . . . . . . 49

4.7.1 Stationary Harmonic maps . . . . . . . . . . . . . . . . . . . 49

4.7.2 Aholomorphic maps between hyperKähler manifolds . . . . . 52

4.8 Aholomorphic maps and harmonic spinors . . . . . . . . . . . 53

5 Generalized Seiberg-Witten equations $\quad 62$

5.1 Generalized Seiberg-Witten Equations . . . . . . . . . . . . . 62

5.2 Modified Seiberg-Witten equations . . . . . . . . . . . . . . . . 64

5.3 Variational Formulation . . . . . . . . . . . . . . . . 66

5.4 A priori estimates . . . . . . . . . . . . . . 70

6 Conclusion $\quad 73$

$\begin{array}{ll}\text { Bibliography } & 75\end{array}$ 


\section{Introduction}

My task, which I am trying to achieve is, by the power of the written word, to make you hear, to make you feel-it is, before all, to make you see.

- Joseph Conrad, The Nigger of the Narcissus and the Secret Sharer

A long standing problem in Differential Geometry has been to understand the classification of smooth manifolds. Classification for compact, simply connected manifolds of dimension $\geq 5$ and for dimension three (Poincarè Conjecture) is known. However, dimension four still remains a mystery. Donaldson, using the Yang-Mills equations, made a successful breakthrough in the 1980's by showing that the moduli spaces of instantons encode non-trivial geometric information of the underlying manifold. But the non-linear nature of the Yang-Mills equations posed some serious technical challenges.

In the fall of 1994, Nathan Seiberg and Edward Witten introduced a set of equations, now known as the Seiberg-Witten equations, that claimed to reproduce the same topological data as the Yang-Mills equations. The Seiberg-Witten invariants were first computed for Kähler manifolds by Witten [Wit94]. Although, again non-linear in nature, these set of equations are technically less demanding. Therefore, it is but natural to explore the variants of these equations in the hope of finding new invariants for four-dimensional manifolds. One such variant was introduced by C.H. Taubes [Tau99] for dimension three and later extended for dimension four by V. Ya. Pidstrygach [Pid04].

In this thesis, we study the non-linear Dirac operator in dimension four and the corresponding generalization of the the Seiberg-Witten equations. The central object for defining the generalization is a hyperKähler manifold. A hyperKähler manifold $\left(M, g_{M}, I_{1}, I_{2}, I_{3}\right)$ is a Riemann manifold of dimension $4 n$ endowed with three complex structures satisfying quaternionic relations and are covariantly constant w.r.t the Levi-Civita connection. These define a family of complex structures 
on $M$ parametrized by $S^{2} \in \mathfrak{I m}(\mathbb{H})$. HyperKähler manifolds belong to a special class of Einstein manifolds and therefore are naturally of interest to physicists. Examples of hyperKähler manifolds include $K 3$-surfaces and the flat spaces $\mathbb{H}^{n}$. One can construct more examples via hyperKähler reduction [HKLR87], which is an analogue of Marsden-Weinstein reduction for symplectic manifolds. Namely, let $G$ be a compact Lie group acting on $M$ by isometries and preserving the hyperKähler structure. Then $G$ preserves $\omega$. We can define a hyperkahler moment map for the $G$-action $\mu_{G}: M \longrightarrow \mathfrak{s p}(1) \otimes \mathfrak{g}^{*}$. For any regular value $b \in \mathfrak{s p}(1) \otimes \mathfrak{g}$, $\mu_{G}^{-1}(b) / G$ is a hyperKähler manifold.

Let $X$ a four dimensional spin-manifold. A spinor bundle on $X$, is a vector bundle associated to the spin-structure on $X$, with fibre $\mathbb{H}$. The idea to define the non-linear generalized Dirac operator, is to replace the fibre of the spinor bundle by a hyperKähler manifold with a permuting action of the group $S p(1)$; i.e an action that permutes the 2-sphere of complex structures on $M$. Generalized spinors are sections of the associated fibre-bundle. The interaction of the $S p(1)$-action with the quaternionic structure on $M$, allows us to define the Clifford multiplication. Composing the covariant derivative on generalized spinors with the Clifford multiplication defines the non-linear generalized Dirac operator, denoted by $\mathscr{D}$.

To define the generalization of the Seiberg-Witten equations, we need an additional $G$-structure $P_{G} \longrightarrow X$, for some compact Lie group $G$ such that $G \curvearrowright M$ is a hyperKähler action. Every connection $\mathrm{B}$ on $P_{G}$ gives an additional twisting of the Dirac operator. Composing a spinor with the moment map $\mu_{G}$ we can construct a self-dual two form using the identification $\mathfrak{s p}(1) \cong \Lambda_{+}^{2} \mathbb{R}^{4}$. The generalized Seiberg-Witten equations in dimension four are defined as:

$$
\left\{\begin{array}{l}
\mathscr{D}_{\mathrm{B}} u=0 \\
F_{\mathrm{B}}^{+}-\mu_{G} \circ u=0
\end{array}\right.
$$

The equations are invariant under the action of the infinite-dimensional gaugegroup $\mathscr{G}$ and hence we get an action of $\mathscr{G}$ the space of solutions to the equations $\mathscr{Z}$. The moduli space is defined as $\mathfrak{M}=\mathscr{Z} / \mathscr{G}$.

Indeed, for the original Seiberg-Witten equations, $G=U(1)$ and $M=\mathbb{H}$, the quadratic map $\mathbb{H} \ni h \longmapsto \frac{1}{2} h i \bar{h} \in \mathfrak{s p}(1)$ is the hyperKähler momentum map for $U(1) \curvearrowright \mathbb{H}$. The moduli space in this case is compact and the key to proving compactness is the $L^{\infty}$-bound on the spinor part of the solution. This is derived 
using the Weitzenböck formula. The invariants derived from the moduli space encodes the geometric information of the underlying four-dimensional manifold $X$. For details, we refer to [Nic], [Mor96] \& [Mar99]. For the generalized SeibergWitten equations, it was proved in [Pid04], that the moduli space is compact for $G=U(1)$ and for a target hyperKähler manifold admitting a certain hyperKähler potential; i.e a real-valued function on $M$ that is simultaneously a Kähler potential for all three complex structures. For a general compact Lie group $G$, if the target hyperKähler manifold is the total space of a Swann bundle, then the $L^{\infty}$-bounds on the spinor part exists [Sch10]. Swann bundles were first introduced by A. Swann [Swa91].

The total space of a Swann bundle can be expressed as a Riemann cone over a Sasakian three-manifold. Hence it admits an action $\mathbb{R}^{+}$, which makes it a suitable candidate to explore the behaviour of the non-linear Dirac operator under the conformal change of metric on the base manifold. In this scenario, we prove in Theorem 4.4.1 that the non-linear generalized Dirac operator exhibits the same behaviour as the linear Dirac operator under the conformal change of metric.

We restrict our attention to analyzing the generalized Seiberg-Witten equations for the case where $M=\mathcal{O}_{n+1}$ is the total space of the Swann bundle over the Wolf space $X(n-1)=\frac{U(n+1)}{S U(2) \times U(n-1) \times U(1)}$ and $G=S U(n+1)$. Advantage is that the space $\mathcal{O}_{n+1}$ is a $U(1)$-hyperKähler reduction of $\mathbb{H}^{n+1} \backslash\{0\}$. [BGM93]

We lift the entire problem to the space $\mathbb{H}^{n+1}$ as follows: let $G=S U(n+1) \times U(1)$ and $\mu_{U(1)}: \mathbb{H}^{n+1} \longrightarrow \mathfrak{s p}(1)^{*}$ denote the hyperKähler momentum map for the action of $U(1) \curvearrowright \mathbb{H}^{n+1}$. The bundle

$$
\mathbb{H}^{n+1} \supset \mu_{U(1)}^{-1}\{0\} \longrightarrow \mu_{U(1)}^{-1}\{0\} / U(1)=\mathcal{O}_{n+1},
$$

is an $S U(n+1)$-equivariant principal bundle. Spinors are now sections of the vector bundle with fibre $\mathbb{H}^{n+1}$ instead of $\mathcal{O}_{n+1}$. The Seiberg-Witten equations are modified accordingly:

$$
\left\{\begin{array}{l}
\mathfrak{D}_{\mathrm{B}} u=0 \\
F_{\mathrm{B}_{S U(n+1)}^{+}}^{+}-\mu_{\mathrm{B}_{S U(n+1)}} \circ u=0 \\
\mu_{U(1)} \circ u=0
\end{array}\right.
$$

where $\mathrm{B}_{S U(n+1)}$ denotes the $S U(n+1)$ component of the connection on $P_{G}$. Using the Weitzenböck formula (Theorem 5.4.1), we obtain the a priori $L^{\infty}$-bound on the 
spinor part of the solution to Eq.(1.0.2) and global $W^{1,2}$-estimates on the spinor and the $S U(n+1)$-component of the connection. 


\title{
2. Preliminaries and notations
}

\author{
"Begin at the beginning," the King said, gravely, \\ "and go on till you come to an end; then stop." \\ - Lewis Carroll, Alice in Wonderland
}

In this chapter we shall review some basic concepts from Spin geometry which we shall need in the further chapters.

\subsection{Fibre bundles}

Thoughout all the chapters, the manifolds in considerations are smooth, paracompact and finite dimensional Riemann manifolds, unless otherwise mentioned.

Definition 2.1.1 (Fibre Bundles). A smooth fibre bundle is a triple $(F, E, X)$ along with a smooth map $\pi: E \longrightarrow X$ called the projection such that given a point $x \in X$, there exists an open neighbourhood $x \in U$ and a diffeomorphism $\gamma_{U}: U \times F \longrightarrow \pi^{-1}(U)$ satisfying $\pi \circ \gamma_{U}=p r_{U}$. E is called the total space of the bundle, $X$ is called the base space and $F$ is called as the fibre of the bundle.

The pair $\left(\gamma_{U}, U\right)$ is called a chart of the bundle and the collection of charts $\left\{\left(\gamma_{i}, U_{i}\right)\right\}_{i \in I}$, for some $I$, such that $\left\{U_{i}\right\}_{i \in I}$ is an open cover of the base $X$ is called a bundle atlas. Given a bundle atlas, let $\left(U_{i}, \gamma_{i}\right)$ and $\left(U_{j}, \gamma_{j}\right)$ be any two bundle charts such that $U_{i} \cap U_{j} \neq \phi$. Then $\gamma_{i} \circ \gamma_{j}^{-1}: U_{i} \cap U_{j} \times F \longrightarrow U_{i} \cap U_{j} \times F$. They define the transition maps $\gamma_{i j}: U_{i} \cap U_{j} \longrightarrow \operatorname{Diff}(\mathrm{F})$, given by: $\gamma_{i} \circ \gamma_{j}^{-1}(e, f)=\left(e, \gamma_{i j}(e) f\right)$ satisfying:

1. $\gamma_{i i}(x)=I d_{F}$

2. $\gamma_{i j} \circ \gamma_{j k} \circ \gamma_{k i}=I d_{F}$. (cocycle condition) 
Definition 2.1.2. (Sections of a Fibre bundle) A smooth map $u: X \longrightarrow E$ is called as a section of the fibre bundle if $\pi \circ s=I d_{X}$.

Definition 2.1.3. (Connections on Fibre bundles) Given a fibre bundle $\pi: E \longrightarrow X$, with a fibre $F$, consider the map $T \pi: T E \longrightarrow T X$. The kernel of this map is a sub-bundle $\mathcal{V}_{E}$ of the tangent bundle $T E$, called the vertical bundle. A connection on the fibre bundle is an assignment of a horizontal $\mathcal{H}_{E}$ distribution such that $T E=\mathcal{H}_{E} \oplus \mathcal{V}_{E}$.

\section{$2.2 \quad$ Principal bundles}

A smooth right action of a Lie group $G$ on a manifold $P$ is a smooth map $G \times P \longrightarrow$ $P$ given by $(g, p) \longrightarrow p \cdot g=: R_{g}(p)$ satisfying

$$
\begin{array}{cr}
(p \cdot h) \cdot g=p \cdot(h g) & \text { for all } h, g \in G \text { and } p \in P \\
e \cdot p=p & \text { for } p \in P
\end{array}
$$

Every element $g \in G$ defines a diffeomorphism $R_{g}: P \longrightarrow P$ and hence the map

$$
G \longrightarrow \operatorname{Diff}(P), \quad g \mapsto R_{g},
$$

where $\operatorname{Diff}(P)$ is the group of diffeomorphisms of $P$, is a group homomorphism. Let $G$ act smoothly and freely on $P$ on the right. Let $X$ denote the quotient of the space by the equivalence relation induced by the $G$ action and $\pi: P \longrightarrow X$, the canonical projection. Then $(G, P, X)$ is a fibre bundle, known as principal bundle and $G$ is called as its structure group. With slight abuse of notation, we shall denote a principal bundle simply by $P$, wherever clear from the context.

Let $\mathfrak{g}$ be the Lie algebra of $G$ and let $\eta \in \mathfrak{g}$. For a smooth, free action of the Lie group on a manifold $P$, the fundamental vector field $K_{\eta}^{P} \in \Gamma(P, T P)$ generated by $\eta$ is given by:

$$
\left.K_{\eta}^{P}\right|_{p}=\left.\frac{d}{d t}(p \cdot \exp (t \eta))\right|_{t=0} \in T_{p} P .
$$

For $g \in G$,

$$
\left.\left(R_{g}\right)_{*} K_{\eta}^{P}\right|_{p}=\left.\frac{d}{d t}(p \cdot \exp (t \eta) g)\right|_{t=0}=\frac{d}{d t}\left(\left.p \cdot g \exp \left(t a d_{g^{-1}}(\eta)\right)\right|_{t=0}=\left.K_{A d_{g^{-1}}(\eta)}^{P}\right|_{p g} .\right.
$$


Also for $x=\pi(p)$ and $T \pi: T P \longrightarrow T X$, we have:

$$
\left.T \pi\left(K_{\eta}^{P}\right)\right|_{p}=T \pi\left(\left.\frac{d}{d t} p \cdot \exp (t \eta)\right|_{t=0}\right)=\left.\frac{d}{d t} \pi(p \cdot \exp (t \eta))\right|_{t=0}=0 .
$$

Since $G$ preserves the fibres, $K_{\eta}^{P}$ is a tangent to the fibres. Since the action is free, the dimension of the fibre is equal to that of $\mathfrak{g}$. Let $\mathcal{V}_{P} \subset T P$ be the space spanned by the fundamental vector fields. Then clearly $\mathcal{V}_{P}=\operatorname{ker}(T \pi)$ and hence $\mathcal{V}_{P}$ is a vertical subspace of $T P$.

\subsubsection{Connections on principal bundles}

A connection on $P$ defines a $G$-invariant distribution $\mathcal{H}_{P}$, known as horizontal distribution on $T P$ such that $T P=\mathcal{H}_{P} \oplus \mathcal{V}_{P}$. This is equivalent to defining a connection one form on $P$ :

Definition 2.2.1. (Connection 1-form) A connection 1-form $A$ on $P$ is a $\mathfrak{g}$ valued 1-form satisfying the following conditions:

1. $\mathrm{A}\left(K_{\eta}^{M}\right)=\eta$ for $\eta \in \mathfrak{g}$

2. $\left(R_{g}\right)^{*} \mathrm{~A}=\operatorname{Ad}\left(g^{-1}\right) \mathrm{A}$, where Ad denotes the adjoint representation of $G$ on $\mathfrak{g}$.

Note that $\mathcal{H}_{P}=\operatorname{ker}(\mathrm{A})$.

\section{Definition 2.2.2. (Curvature of a connection)}

The curvature of a connection 1-form $\mathrm{A}$ is a $G$-equivariant, $\mathfrak{g}$-valued 2 -form $F_{\mathrm{A}}$ defined by

$$
F_{\mathrm{A}}=d \mathrm{~A}+\frac{1}{2}[\mathrm{~A}, \mathrm{~A}] \in \Omega^{2}(P, \mathfrak{g})_{h o r}^{G},
$$

where the subscript "hor" refers to the fact that the curvature vanishes on vertical vector fields.

\subsubsection{Connections on Associated bundles}

Let $M$ be a manifold with a smooth left action of $G$. Then the fibre bundle associated to $P$ with a fibre $M$ is defined to be:

$$
\mathrm{M}:=P \times_{G} M \stackrel{\pi_{\mathrm{M}}}{\longrightarrow} X
$$


where $\pi_{\mathrm{M}}([p, m])=\pi(p)$. Given a connection $\mathrm{A}$ on $P$, it induces a connection on $\mathrm{M}$ as follows: At a point $[p, m] \in \mathrm{M}$ the vertical space is isomorphic to $T_{m} M$, whereas the horizontal space is given by $\left.\mathcal{H}_{\mathrm{M}}\right|_{[p, m]}=\left(\left.\mathcal{H}_{P}\right|_{p} \oplus\{0\}\right) / G$.

We have a canonical isomorphism between the spaces $C^{\infty}(P, M)^{G}$ and $\Gamma(X, \mathrm{M})$ given by

$$
\begin{gathered}
C^{\infty}(P, M)^{G} \longrightarrow \Gamma(X, \mathrm{M}) \\
u \longmapsto s_{u} \text { where } s_{u}(x)=[p, u(p)] \text { for } p \in \pi^{-1}(x) .
\end{gathered}
$$

Given a connection $\mathrm{A}$ on $P$, the covariant derivative of a section $u \in C^{\infty}(P, M)^{G}$ is given by

$$
D_{\mathrm{A}} u=T u \circ \operatorname{pr}_{\mathcal{H}_{\mathrm{A}}}=T u+\left.K_{\mathrm{A}}^{M}\right|_{u} \in \operatorname{Hom}(T P, T M)_{h o r}^{G},
$$

where $\mathcal{H}_{\mathrm{A}}$ is the horizontal sub-bundle defined by $\mathrm{A}$.

\subsection{Vector Bundle}

Definition 2.3.1. Vector Bundle Let $X$ be a smooth manifold. A fibre bundle $\pi_{E}: E \longrightarrow X$ is said to be a vector bundle if the fibre is a $\mathbb{K}$-vector space, where $\mathbb{K}=\mathbb{R}$ or $\mathbb{C}$, and the transition maps lie in $\operatorname{Aut}_{\mathbb{K}}(V)$.

Given a principal $G$-bundle $P$ over $X$, let $V$ be a $G$-representation. Then the associated fibre bundle $P \times_{G} V \longrightarrow X$ is a vector bundle. On the other hand, given a rank n vector bundle $\pi_{E}: E \longrightarrow X$, let $\pi_{P}: P_{G L(n, \mathbb{K})} \longrightarrow X$ denote the bundle of frames in $E$. Indeed, for $x \in X$ and $p \in \pi_{P}^{-1}(x), p$ is a $\mathbb{K}$-linear isomorphism $p: \mathbb{K} \longrightarrow T_{x} X$. Then $E=P_{G L(n, \mathbb{K})} \times_{G L(n, \mathbb{K})} \mathbb{K}^{n}$.

Given $u \in C^{\infty}\left(P_{G L(n, \mathbb{K})}, \mathbb{K}^{n}\right)^{G L(n, \mathbb{K})}$ and a connection $\mathrm{A}$ on $P_{G L(n, \mathbb{K})}$, the induced covariant derivative on the associated vector bundle is given by:

$$
D_{\mathrm{A}} u=T u+\mathrm{A} \cdot u \text {. }
$$

\subsection{Action of the gauge group}

\subsubsection{Gauge Group}

Let $P \stackrel{\pi}{\rightarrow} X$ be a principal $G$-bundle over $X$. An automorphism of $P$ is a $G$ equivariant diffeomorphism $\beta: P \longrightarrow P$ such that $\pi \circ \beta=\pi$. The set of all the 
automorphisms of $P$ forms a group called gauge group, denoted by $\mathcal{G}(P)$. The elements of the gauge group are called gauge transformations. Given a gauge transformation $\beta$, it induces a $G$-equivariant map $g: P \longrightarrow G$, defined by $\beta(p)=$ $p g(p)$ (Note that the group $G$ acts on itself by conjugation). Conversely, given any $G$-equivariant map $g: P \longrightarrow G$, it defines a gauge transformation $\beta$, given by $\beta(p)=p g(p)$. Thus we have an isomorphism

$$
C^{\infty}(P, G)^{G} \cong \mathcal{G}(P)
$$

Let $\mathcal{A}(P)$-denote the space of all connections on $P$. If $\beta \in \mathcal{G}(P)$ and $A \in \mathcal{A}(P)$, then clearly $\beta^{*} \mathrm{~A} \in \mathcal{A}(P)$. Thus we have a right action of the gauge group on the space of connections.

Definition 2.4.1. (Maurer-Cartan one form) The Maurer-Cartan 1-form is a left-invariant, $\mathfrak{g}$-valued 1-form on $G$ defined by $\Theta(w)=T L_{h^{-1}}(w)$ for $h \in G$ and $w \in T_{h} G$ and $L_{*}$ denotes the left action of $G$ on itself.

If $G$ is a matrix group so that $G \subset G L(n)$, then the Maurer Cartan form can be written as $\Theta=g^{-1}(d g)$, where $d g: T_{g} G \longrightarrow \mathfrak{g l}(n)$ is the inclusion.

Proposition 2.4.1 ([Bau09]). For $\beta \in \mathcal{G}(P)$, let $g$ denote the corresponding element in $C^{\infty}(P, G)^{G}$. Then,

$$
\beta^{*} \mathrm{~A}=A d_{g^{-1}(\cdot)} \mathrm{A}+g^{*} \Theta
$$

where $\Theta$ denotes the Maurer-Cartan 1-form on $G$. If $F_{\mathrm{A}}$ denotes the curvature of the connection $\mathrm{A}$, then

$$
\beta^{*} F_{\mathrm{A}}=F_{\beta^{*} \mathrm{~A}}=A d_{g^{-1}}\left(F_{\mathrm{A}}\right) .
$$

In terms of the covariant derivatives on the associated (finite dimensional) vector bundle, for a section $s \in C^{\infty}(P, V)^{G}$ of the vector bundle, we have,

$$
D_{\beta^{*} \mathrm{~A}} s=\beta^{-1}\left(D_{\mathrm{A}}(\beta(s))\right) .
$$




\subsection{Elements of Spin Geometry}

\subsubsection{Clifford algebras and Spin Groups}

Let $V$ be a finite dimensional real vector space endowed with a quadratic form $q$. Consider the tensor algebra generated by $V$

$$
T(V)=\bigoplus_{n \geq o} \underbrace{V \otimes V \otimes \cdots \otimes V}_{n-\text { times }}
$$

This is an associative unital algebra.

Consider the two-sided ideal $\mathcal{I}(V, q)$ generated by all the elements of the form $v \otimes v+q(v, v) \cdot 1$ for $v \in V$. Then the Clifford algebra associated to $V$ w.r.t $q$ is defined as $\mathcal{C} l(V, q):=T(V) / \mathcal{I}(V, q)$. Clifford algebra satisfies the universal property, namely, if $\mathfrak{B}$ is another unital associative real algebra and if there exists a linear map $f: V \longrightarrow \mathfrak{B}$ satisfying $f(v) \otimes f(v)+q(v, v) \cdot 1_{\mathfrak{B}}=0$, then there exists a unique morphism of algebras such that the following diagram commutes:

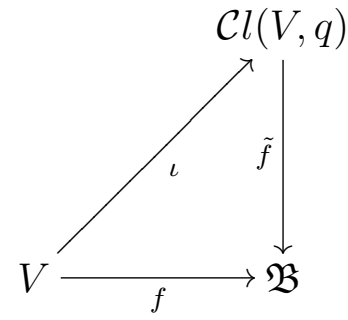

where, $\iota$ denotes the canonical inclusion of $V$ into $\mathcal{C l}(V, q)$.

Note that if $q \equiv 0$, then, $\mathcal{C} l(V, 0)=\Lambda^{*} V$. For $q$ non-degenerate, $\mathcal{C} l(V, q)$ and $\Lambda^{*}(V)$ are canonically isomorphic as vector spaces, the isomorphism being given by

$$
v_{1} \cdot v_{2} \cdots v_{n} \mapsto v_{1} \wedge v_{2} \cdots \wedge v_{n}
$$

where $\left\{v_{1}, v_{2}, \cdots v_{n}\right\}$ is the orthonormal basis of $V$ and the operation "." denotes the multiplication in the Clifford algebra. For simplicity, we shall write $v \cdot w \equiv v w$.

Example 2.5.1. (Clifford Algebras) Denote by $\mathcal{C} l_{n}$, the Clifford algebra of $\left(\mathbb{R}^{n}, q_{s t}\right)$, where $q_{s t}$ is the standard inner product on $\mathbb{R}^{n}$.

1. $\mathcal{C} l_{2} \cong \mathbb{H}$ with $1 \mapsto 1, e_{1} \mapsto i, e_{2} \mapsto j$ and $e_{1} e_{2} \mapsto k$ 
2. $\mathcal{C} l_{3} \cong \mathbb{H} \oplus \mathbb{H}$. Using lemma (2.5.1) stated below, it suffices to note that $\mathcal{C} l_{3}^{\text {even }} \cong \mathcal{C} l_{2}$ and $1 \mapsto 1, e_{1} e_{3} \mapsto i, e_{2} e_{3} \mapsto j, e_{1} e_{2} \mapsto k$.

3. $\mathcal{C} l_{4} \cong M_{2}(\mathbb{H})$ where, $M_{2}(\mathbb{H})$ denotes the set of $2 \times 2$ matrices with quaternionic entries. Identify $\mathbb{R}^{4} \cong \mathbb{H}$. This gives an embedding of $\mathbb{R}$ into $M_{2}(\mathbb{H})$ as follows:

$$
\mathbb{R}^{4} \cong \mathbb{H} \ni x \longmapsto\left(\begin{array}{cc}
0 & -\bar{x} \\
x & 0
\end{array}\right) .
$$

This extends to a morphism of algebras and one can check that this is infact an isomorphism.

The involution map $\alpha: V \longrightarrow V$ given by $\alpha(v)=-v$, defines a $\mathbb{Z}_{2}$-grading of the Clifford algebra

$$
\mathcal{C l}(V, q)=\mathcal{C} l^{0}(V, q) \oplus \mathcal{C} l^{1}(V, q),
$$

corresponding respectively to the eigenvalues \pm 1 of $\alpha$. Under the isomorphism 2.5.1 the decompisition corresponds to the $\mathbb{Z}_{2}$-grading $\Lambda^{*} V=\Lambda^{\text {even }} V \oplus \Lambda^{\text {odd }} V$. Note here that $\mathcal{C} l^{0}(V, q)$ is a sub-algebra of $\mathcal{C} l(V, q)$.

A group of multiplicative units in Clifford algebra is the set

$$
\mathcal{C} l^{*}(V, q)=\left\{\psi \in \mathcal{C} l(V, q) \mid \exists \psi^{-1} \text { such that } \psi \psi^{-1}=\psi^{-1} \psi=1\right\} .
$$

The group $\operatorname{Pin}(V, q) \subset \mathcal{C} l^{*}(V, q)$ is a group, generated by the set of all the units $v \in V \subset \mathcal{C l}(V, q)$ such that $q(v)= \pm 1$. We define the group $\operatorname{Spin}(V, q)$ to be $\operatorname{Pin}(V, q) \cap \mathcal{C} l^{0}(V, q)$.

The group $\mathcal{C} l^{*}(V, q)$ acts on the Clifford algebra by a twisted adjoint action.

$$
\begin{gathered}
A d: \mathcal{C} l^{*}(V, q) \longrightarrow \operatorname{Aut}(\mathcal{C l}(V, q)) \\
\operatorname{Ad}_{\psi}(x)=\alpha(\psi) x \psi^{-1}
\end{gathered}
$$

Restricting to the group $\operatorname{Spin}(V, q)$, we get the adjoint action of $\operatorname{Spin}(V, q)$ on $\mathcal{C l}(V, q)$. The action preserves the subspace $V \in \mathcal{C l}(V, q)$ and therefore gives a real representation of the $\operatorname{Spin}(V, q)$ group. We have the following short-exact sequence:

$$
0 \longrightarrow \mathbb{Z}_{2} \longrightarrow \operatorname{Spin}(V, q) \stackrel{\lambda}{\longrightarrow} S O(V, q) \longrightarrow 0
$$

where $\lambda$ denotes the induced group action. When $V=\mathbb{R}^{n}$, for $n \geq 3$, we define $\operatorname{Spin}(n):=\operatorname{Spin}\left(\mathbb{R}^{n}, q_{s t}\right)$, where $q_{s t}$ is the standard inner product on $\mathbb{R}^{n}$. The group $\operatorname{Spin}(n)$ is a simply connected and hence the universal cover of $S O(n)$. 
Example 2.5.2. 1. In case of dimension three, using the universality property of the group $\operatorname{Spin}(3)$, one can prove that $\operatorname{Spin}(3) \cong S p(1)$. Indeed, consider the identification $\mathbb{R}^{3} \cong \mathfrak{I m}(\mathbb{H})$. Then we have the homomorphism given by

$$
\begin{gathered}
S p(1) \longrightarrow S O(3) \\
q \longmapsto a_{q} \text { where } a_{q}(v)=q v q^{-1}, v \in \mathfrak{I m}(\mathbb{H}) .
\end{gathered}
$$

The kernel of this map is $\{ \pm 1\}$. Sp(1) being simply connected, this implies $\operatorname{Sp}(1) \cong \operatorname{Spin}(3)$.

2. We can argue similarly for dimension four. Identify $\mathbb{R}^{4} \cong \mathbb{H}$, we have the following homomorphism:

$$
\begin{gathered}
S p(1) \times S p(1) \longrightarrow S O(4) \\
q=\left(q_{+}, q_{-}\right) \longmapsto a_{q} \text { where } a_{q}(v)=q_{+} v q_{-}^{-1}, v \in \mathbb{H} .
\end{gathered}
$$

The kernel of the map is $\{(1,1),(-1,-1)\}$. Since $S p(1) \times S p(1)$ is simply connected, we deduce that $S p(1) \times S p(1) \cong \operatorname{Spin}(4)$. We distinguish the two copies of $S p(1)$ by + and - , where the corresponding actions given above.

Lemma 2.5.1 ([LM89] Chapter I, Thm. 3.7). Denote by $\mathcal{C} l_{n}$, the Clifford algebra of $\mathbb{R}^{n}$. The map $\mathbb{R}^{n} \ni v \mapsto v e_{n+1} \in \mathcal{C} l_{n+1}^{0}$ induces an isomorphism,

$$
\mathcal{C} l_{n} \cong \mathcal{C} l_{n+1}^{0}
$$

We shall restrict henceforth to the case $V=\mathbb{R}^{n}$. Choose an orientation of $\mathbb{R}^{n}$ and let $\left\{e_{1}, e_{2}, \cdots e_{n}\right\}$ be any positively oriented orthonormal basis w.r.t the standard inner product on $\mathbb{R}^{n}$. Then the associated oriented volume element in the Clifford algebra is defined by $\omega=e_{1} e_{2} \cdots e_{n}$. This is independent of the choice of an oriented orthonormal frame and satisfies $\omega^{2}=(-1)^{\frac{n(n+1)}{2}}$. For any $v \in \mathbb{R}^{n}, \quad v \omega=(-1)^{n-1} \omega v$. Consequently, $\omega$ is central if $n$ is odd. If $n$ is even, then for any $\gamma \in \mathcal{C} l_{n}, \gamma \omega=\omega \tau(\gamma)$, where $\tau$ denotes the parity operator.

Lemma 2.5.2 ([LM89] Chapter I, Lemma 3.4). If $\omega^{2}=1$, define

$$
\varepsilon_{ \pm}=\frac{1 \mp \omega}{2}
$$

Then the following relations are satisfied: 


$$
\begin{gathered}
\varepsilon_{+}+\varepsilon_{-}=1 \\
\left(\varepsilon_{ \pm}\right)^{2}=\varepsilon_{ \pm} \\
\varepsilon_{+} \varepsilon_{-}=\varepsilon_{-} \varepsilon_{+}=0 .
\end{gathered}
$$

Suppose now that $\omega$ satisfies $\omega^{2}=1$. If $n$ is odd, then the Clifford algebra decomposes into a direct sum of isomorphic sub-algebras $\mathcal{C} l_{n}=\left(\mathcal{C} l_{n}\right)_{+} \oplus\left(\mathcal{C l}_{n}\right)_{-}$, corresponding respectively to the eigen-values \pm 1 of $\omega$. If $n$ is even, $\omega$ is central in the sub-algebra $\mathcal{C} l_{n}^{0}$. Hence $\mathcal{C} l_{n}^{0}$ decomposes as $\left(\mathcal{C} l_{n}^{0}\right)_{+} \oplus\left(\mathcal{C} l_{n}^{0}\right)_{-}$analogously.

Consider the three dimensional Clifford algebra $\mathrm{Cl}_{3}$. The volume element $\omega$ satisfies $\omega^{2}=1$ and is central in $\mathrm{Cl}_{3}$. Hence $\mathcal{C l}_{3}$ decomposes as $\left(\mathrm{Cl}_{3}\right)_{+} \oplus\left(\mathrm{Cl}_{3}\right)_{-}$. Indeed, the decomposition of $\mathrm{Cl}_{3}$ as two copies of quaternions (cf. Example 2.5.1) corresponds to the decompostion $\mathcal{C l}_{3}=\left(\mathcal{C l}_{3}\right)_{+} \oplus\left(\mathrm{Cl}_{3}\right)_{-}$. Therefore $\left(\mathrm{Cl}_{3}\right)_{ \pm} \cong \mathbb{H}$.

In the development further, we shall need the following lemma:

Lemma 2.5.3. [Pid13] Let $n$ be even and $\omega$ be the oriented volume element of $\mathrm{Cl}_{n}$ and $\varepsilon_{ \pm}$be as defined in Lemma (2.5.2). Then we have the following identities

$$
\varepsilon_{+} \mathcal{C} l_{n} \varepsilon_{+}=\left(\mathcal{C} l_{n}^{0}\right)_{+} \quad \text { and } \quad \varepsilon_{-} \mathcal{C} l_{n} \varepsilon_{+}=\varepsilon_{-} \mathcal{C} l_{n}^{1}=\mathcal{C} l_{n}^{1} \varepsilon_{+}
$$

Proof : Let $\gamma \in \mathcal{C} l_{n}$. The $\varepsilon_{+} \gamma \varepsilon_{+}=\frac{1}{4}(1-\omega) \gamma(1-\omega)$. Let $\tau$ denote the parity operator. Then

$$
\begin{aligned}
\frac{1}{4}(1-\omega) \gamma(1-\omega) & =\frac{1}{4}(1-\omega)(\gamma-\omega \tau(\gamma)) \\
& =\frac{1}{4}((1-\omega) \gamma-\omega \tau(\gamma)+\tau(\gamma))=\frac{1}{4}(1-\omega)(\gamma+\tau(\gamma))=\frac{1}{2}(1-\omega) \gamma^{0} .
\end{aligned}
$$

The second identity can be proved in a similar manner.

\subsubsection{Clifford modules and Spin representations}

Consider a vector space $(V, q)$ over a field $\mathbb{K}$, where $q$ is a quadratic form on $V$.

Definition 2.5.1. A a $\mathcal{C l}(V, q)$-module over $\mathbb{K}$ is a $\mathbb{K}$-algebra homorphism

$$
\rho: \mathcal{C l}(V, q) \longrightarrow \operatorname{End}_{K}(\mathrm{~W})
$$

into the algebra of all the $K$-linear transformations of a finite dimensional $K$-vector space W. 
We are mainly interested in the case $\mathbb{K}=\mathbb{R}$ and $V=\mathbb{R}^{n}$ endowed with the standard inner product.

Proposition 2.5.1 ([LM89] Chapter I, Prop. 5.10). Let $n=4 m$ and let $\mathrm{W}$ be an irreducible $\mathcal{C} l_{n}$-representation. Then $\omega^{2}=1$ and each of the subspaces $\mathrm{W}_{ \pm}$is invariant under the Clifford sub-algebra $\mathcal{C} l_{n}^{0}$. Under the isomorphism $\mathcal{C} l_{n-1} \cong \mathcal{C} l_{n}^{0}$, these correspond to two distinct irreducible representations of $\mathcal{C} l_{n-1}$.

Definition 2.5.2. A $\mathbb{Z}_{2}$-graded $\mathcal{C} l_{n}$-module is a module with a $\mathbb{Z}_{2}$-grading $\mathrm{W}=$ $\mathrm{W}^{0} \oplus \mathrm{W}^{1}$ such that $\mathcal{C} l_{n}^{i} \mathrm{~W}^{j} \subseteq \mathrm{W}^{(i+j) \bmod (2)}$, for $0 \leq i, j \leq 1$.

Proposition 2.5.2. [ABS64] There exists a bijection from the category of nongraded $\mathcal{C} l_{n-1}$-modules and $\mathbb{Z}_{2}$-graded $\mathcal{C} l_{n}$-modules. Indeed if $\mathrm{W}^{0} \oplus \mathrm{W}^{1}$ is a $\mathbb{Z}_{2}$-graded module over $\mathcal{C} l_{n}$, then $\mathrm{W}^{0}$ is a module over $\mathcal{C} l_{n}^{0} \cong \mathcal{C} l_{n-1}$.

On the other hand, given a non-graded module $W_{n}^{0}$ over $\mathcal{C} l_{n}^{0} \cong \mathcal{C} l_{n-1}$, we get the $\mathbb{Z}_{2}$-graded module over $\mathcal{C} l_{n}$ by defining $\mathrm{W}=\mathcal{C} l_{n} \otimes_{\mathcal{C} l_{n}^{0}} \mathrm{~W}^{0}$. The left multiplication of $\mathcal{C} l_{n}$ on $\mathcal{C} l_{n}$, makes $\mathrm{W}$ into a $\mathbb{Z}_{2}$-graded module.

Each Clifford module gives rise to a representation of the Spin group.

Definition 2.5.3. (Representation of $\operatorname{Spin}(n)$ ) Let $\mathrm{W}$ be an irreducible $\mathcal{C} l_{n^{-}}$ module. A real spinor representation is a homomorphism $\zeta: \operatorname{Spin}(n) \longrightarrow \operatorname{End}(\mathrm{W})$ obtained by restricting $\zeta: \mathcal{C} l_{n} \longrightarrow \operatorname{End}(\mathrm{W})$ to $\operatorname{Spin}(n) \subset \mathcal{C} l_{n}^{0} \subset \mathcal{C} l_{n}$.

Once again, consider the three dimensional Clifford algebra $\mathcal{C} l_{3} \cong \mathbb{H} \oplus \mathbb{H}$. Then $\mathcal{C} l_{3}^{0} \cong\{(h, h) \mid h \in Q\} \subset \mathbb{H} \oplus \mathbb{H}$ and $\mathcal{C} l_{3}^{1} \cong\{(h,-h) \mid h \in Q\} \subset \mathbb{H} \oplus \mathbb{H}$. Restricting to the $\operatorname{Spin}(3) \in \mathcal{C} l_{3}^{0}$, we obtain the spinor representation, denoted by $\mathbb{V}$. Then $\mathcal{C} l_{4} \otimes_{\mathcal{C l}} \mathbb{V}$ is a $\mathcal{C} l_{4}$-module with the left action of $\mathcal{C} l_{4}$ on itself (cf. Lemma 2.5.1 and Prop. 2.5.2). Now consider the irreducible $\mathcal{C} l_{4} \cong M_{2}(\mathbb{H})$-module $\mathbb{H}^{2}$. Restricting this to $\mathcal{C} l_{4}^{0}$ gives two distinct irreducible $\mathcal{C} l_{4}^{0}$-modules.

$$
\begin{aligned}
\mathbb{H} \oplus \mathbb{H} \cong \mathcal{C} l_{3} \cong \mathcal{C} l_{4}^{0} \underset{\mathcal{C} l_{4} \cong M_{2}(\mathbb{H})}{\left(h_{1}, h_{2}\right)} \longmapsto\left(\begin{array}{cc}
h_{1} & 0 \\
0 & h_{2}
\end{array}\right)
\end{aligned}
$$

Restricting further to $\operatorname{Spin}(4) \subset \mathcal{C} l_{4}^{0}$, these give the spinor representation. Homologous to the product structure of $\operatorname{Spin}(4) \cong S p(1)_{+} \times S p(1)_{-}$, we have two distinct representations $\mathbb{W}^{+}$and $\mathbb{W}^{-}$. 
Lemma 2.5.4. [LM89] The two $\mathcal{C l}_{4}$-representations are isomorphic, the isomorphism being given by

$$
\begin{gathered}
\varrho: \mathbb{H}^{2} \longrightarrow \mathcal{C} l_{4} \otimes_{\mathcal{C} l_{4}^{0}} \mathbb{V} \\
(v, w) \longmapsto 1 \otimes v+e_{0} \otimes w .
\end{gathered}
$$

Restriction to the group Spin(4) induces an isomorphism Spin(4)-representations:

$$
\mathbb{W}^{+} \oplus \mathbb{W}^{-} \cong \mathcal{C} l_{4} \otimes_{\mathcal{C} l_{4}^{0}} \mathbb{V}
$$

Let $\mathbb{H} \longrightarrow \mathbb{R}^{4}$ be the standard identification given by $h \longmapsto e_{h}$. The subalgebra $\left(\mathcal{C} l_{4}^{0}\right)_{+}=\left\{e_{0} e \varepsilon_{+} \mid e \in \mathbb{R}^{4}\right\}$ is a free left $\left(\mathcal{C} l_{4}^{0}\right)_{+}$-module with generator $\varepsilon_{+}$. The isomorphism $\lambda: \mathbb{H} \longrightarrow\left(\mathcal{C l}_{4}^{0}\right)_{+}$, given by $h \longmapsto e_{0} e_{-\bar{h}} \varepsilon_{+}$, defines a complex structure on $\left.(\mathcal{C l})_{4}^{0}\right)_{+}$:

$$
\begin{aligned}
& 1 \longmapsto \varepsilon_{+} i \longmapsto J_{1}=\frac{e_{0} e_{1}+e_{2} e_{3}}{2} \\
& j \longmapsto J_{2}=\frac{e_{0} e_{2}-e_{1} e_{3}}{2} \quad k \longmapsto J_{3}=\frac{e_{0} e_{3}+e_{1} e_{2}}{2} .
\end{aligned}
$$

The odd part of the Clifford module $\mathcal{C} l_{4} \varepsilon_{+}$, given by,

$$
\varepsilon_{-} \mathcal{C} l_{4} \varepsilon_{+}=\left\{e \varepsilon_{+} \mid e \in \mathbb{R}^{4}\right\}
$$

is a free left $\left(\mathrm{Cl}_{4}^{0}\right)_{+}$-module with the generator $e_{0} \varepsilon_{+}$.

Lemma 2.5.5. [Pid13] The Clifford multiplication maps

$$
\begin{gathered}
\mathbb{R}^{4} \otimes \varepsilon_{+}\left(\mathcal{C} l_{4}\right) \varepsilon_{+} \longrightarrow \varepsilon_{-}\left(\mathcal{C} l_{4}\right) \varepsilon_{+} \\
e \otimes \varepsilon_{+} \longmapsto e_{0} \varepsilon_{+} \lambda_{R_{e_{0}}}(e)
\end{gathered}
$$

Proof : For any $e \in \mathbb{R}^{4}$, the Clifford multiplication maps

$$
\mathbb{R}^{4} \otimes \varepsilon_{+}\left(\mathcal{C} l_{4}\right) \varepsilon_{+} \ni e \otimes \varepsilon_{+} \mapsto e \varepsilon_{+} \in \varepsilon_{-} \mathcal{C} l_{4} \varepsilon_{+}=\left\{e \varepsilon_{+} \mid e \in \mathbb{R}^{4}\right\} .
$$

But

$$
\begin{aligned}
e \varepsilon_{+} & =-e_{0}^{2} e \varepsilon_{+}=e_{0}\left(-e_{0}\right) e \varepsilon_{+}^{2}=e_{0} \varepsilon_{+} \underbrace{\left(-e_{0} e e_{0}^{-1}\right)}_{R_{e_{0}}(e)} e_{0} \varepsilon_{+} \\
& =e_{0} \varepsilon_{+} R_{e_{0}}(e) e_{0} \varepsilon_{+}=e_{0} \varepsilon_{+} \lambda_{R_{e_{0}}}(e)
\end{aligned}
$$


Observe that $R_{e_{0}}\left(e_{0}\right)=-e_{0}$ and $R_{e_{0}}\left(e_{0}^{\perp}\right)=-e_{0} e_{0}^{\perp} e_{0}^{-1}=e_{0}^{\perp}$. Therefore, in quaternionic notation, $e_{h} \varepsilon_{+}=e_{0} \varepsilon_{+} \lambda_{-}$.

Given a $\mathcal{C} l_{4}^{0}$-module $W^{0}$, by Proposition 2.5.2, we can define the Clifford module $W=\mathcal{C} l_{4} \otimes_{\mathcal{C l}_{4}^{0}} W^{0}$. Since $\varepsilon_{-} \cdot W^{0}=0$,

$$
\begin{aligned}
W & =\mathcal{C} l_{4} \varepsilon_{+} \otimes_{\left(\mathcal{C} l_{4}^{0}\right)_{+}} W^{0} \\
& =\left(\varepsilon_{-} \mathcal{C} l_{4} \varepsilon_{+} \otimes_{\left(\mathcal{C} l_{4}^{0}\right)_{+}} W^{0}\right) \oplus\left(\varepsilon_{+} \mathcal{C} l_{4} \varepsilon_{+} \otimes_{\left(\mathcal{C l} l_{4}^{0}\right)_{+}} W^{0}\right) \\
& =\left(\varepsilon_{-} \mathcal{C} l_{4} \varepsilon_{+} \otimes_{\left(\mathcal{C} l_{4}^{0}\right)_{+}} W^{0}\right) \oplus W^{0}
\end{aligned}
$$

\subsubsection{Clifford multiplication}

\section{Definition 2.5.4. (Clifford Multiplication)}

Let $\mathrm{W}$ be a $\mathcal{C} l_{n}$-module and $\rho$ be the map $\rho: \mathcal{C} l_{n} \longrightarrow \operatorname{End}(\mathrm{W})$. Consider the restriction of the action of $\mathcal{C} l_{n}$ on $W$ to $\mathbb{R}^{n}$. Then, Clifford multiplication is the map of $\operatorname{Spin}(n)$-representations

$$
\begin{aligned}
& \mathfrak{c}: \mathbb{R}^{n} \otimes \mathbf{W} \longrightarrow \mathbf{W} \\
& \mathfrak{c}(x \otimes v)=\rho(x) \cdot v
\end{aligned}
$$

For simplicity, denote the Clifford multiplication by $x \cdot v$ for $x \in \mathbb{R}^{n}$ and $v \in W$.

Example 2.5.3. In dimension four, identify $\mathbb{R}^{4} \cong \mathbb{H}$ by mapping the standard oriented basis $\left(e_{1}, e_{2}, e_{3}, e_{4}\right)$ of $\mathbb{R}^{4}$ to the basis $(1, \bar{i}, \bar{j}, \bar{k})$ of $\mathbb{H}$. Define the Clifford multiplication to be the map

$$
\begin{aligned}
\mathfrak{m}: \mathbb{R}^{4} \cong \mathbb{H} & \longrightarrow \operatorname{End}\left(\mathbb{W}^{+} \oplus \mathbb{W}^{-}\right) \\
h & \longmapsto\left(\begin{array}{cc}
0 & -\bar{h} \\
h & 0
\end{array}\right)
\end{aligned}
$$

It is easily verified that $\mathfrak{m}(h)^{2}=-g_{\mathbb{R}^{4}}(h, h) i d_{\mathbb{W}^{+} \oplus \mathbb{W}^{-}}$.

Note 2.5.1. The Clifford multiplication maps $\mathbb{W}^{+} \oplus \mathbb{W}^{-} \longmapsto \mathbb{W}^{-} \oplus \mathbb{W}^{+}$.

\subsubsection{Spin structures}

Let $X$ be a $n$-dimensional Riemann manifold. Let $E \longrightarrow X$ be an oriented vector bundle over $X$ with a fibre $V$. Let $P_{S O(V)}$ denote the principal frame bundle over 
$X$ associated to $E$. Then a $\operatorname{Spin}(V)$-structure on $E$ is a principal $\operatorname{Spin}(V)$-bundle over $X$, denoted by $Q_{\operatorname{Spin}(V)}$, which is an equivariant double cover

$$
\Psi: Q_{\operatorname{Spin}(V)} \longrightarrow P_{S O(V)}
$$

i.e. $\Psi(q g)=\Psi(q) \lambda(g)$, for $g \in \operatorname{Spin}(V)$ and $q \in Q_{\operatorname{Spin}(V)}$.

Proposition 2.5.3 ([LM89] Chapter II, Thm. 1.7). Let $E \longrightarrow X$ be an oriented Riemann vector bundle over $X$. Then the existence of a Spin structure on $E$ is guaranteed iff the second Steifel-Whitney class $w_{2}(E)=0$.

The obstruction to the lift can be understood in terms of Čech cohomology class. The bundle $P_{S O(V)} \longrightarrow X$ is determined by a trivializing open cover $\left\{\mathcal{U}_{\alpha}\right\}_{\alpha \in \mathrm{J}}$, for some index set J, along with transition maps $g_{\alpha \beta}: \mathcal{U}_{\alpha} \cap \mathcal{U}_{\beta} \longrightarrow S O(V)$.

Let $\left\{\mathcal{U}_{\alpha}\right\}$ denote a trivializing open cover for $P_{S O(V)}$ such that $\mathcal{U}_{\alpha} \cap \mathcal{U}_{\beta}$ is contractible. Let $\mathrm{g} \in \check{H}^{1}(X, S O(V))$ and let $g_{\alpha \beta}: \mathcal{U}_{\alpha} \cap \mathcal{U}_{\beta} \longrightarrow S O(V)$ be any representative of $g$. Since $\mathcal{U}_{\alpha} \cap \mathcal{U}_{\beta}$ is contractible, $g_{\alpha \beta}$ can be lifted to a smooth map

$\tilde{g}_{\alpha \beta}: \mathcal{U}_{\alpha} \cap \mathcal{U}_{\beta} \longrightarrow \operatorname{Spin}(V)$. By the exactness of the sequence :

$$
0 \longrightarrow \mathbb{Z}_{2} \longrightarrow \operatorname{Spin}(V) \stackrel{\lambda}{\longrightarrow} S O(V) \longrightarrow 0
$$

we get that $\eta_{\alpha \beta \gamma}:=\tilde{g}_{\alpha \beta} \tilde{g}_{\beta \gamma} \tilde{g}_{\beta \gamma}: \mathcal{U}_{\alpha} \cap \mathcal{U}_{\beta} \cap \mathcal{U}_{\gamma} \longrightarrow\{1,-1\}$. In other words, $\left\{\eta_{\alpha \beta \gamma}\right\}$ is a Čech cocycle and represents a cohomology class $w_{2}(E) \in H^{2}\left(X, \mathbb{Z}_{2}\right)$.

The cohomology class $w_{2}(E)$ is known as the second Stiefel-Whitney class of E.

Definition 2.5.5. An oriented, $n$-dimensional Riemann manifold $X$ is a Spin manifold iff $w_{2}(T X)=0$.

\subsubsection{Spinor bundles and Dirac operators}

Let $\mathrm{W}$ be a $\mathcal{C} l_{n}$-representation. Then by restriction, $\mathrm{W}$ is also a $\operatorname{Spin}(n)$-representation. Let $X$ be a Spin-maifold. A Spinor bundle is the associated vector bundle $\mathbb{W}:=Q_{\operatorname{Spin}(n)} \times_{\operatorname{Spin}(n)} \mathrm{W}$. Spinors are then defined to be smooth sections of $\mathbb{W}$. Since we have a canonical isomorphism between the spaces $C^{\infty}\left(Q_{\operatorname{Spin}(n)}, \mathrm{W}\right)^{\operatorname{Spin}(n)}$ and $\Gamma(X, \mathbb{W})$, we can equivalently define spinors to be elements of $C^{\infty}\left(Q_{\operatorname{Spin}(n)}, \mathbf{W}\right)^{\operatorname{Spin}(n)}$. 
Example 2.5.4. In dimension four, we have two irreducible representations of $\operatorname{Spin}(4) \cong S p(1)_{+} \times S p(1)_{-}$, namely $\mathbb{W}^{+}$and $\mathbb{W}^{-}$. The spinor bundles corresponding to the two representations are denoted by $\mathbb{W}^{+}$and $\mathbb{W}^{-}$. The sections of these are called positive and negative spinors, respectively.

Let $X$ be an $n$-dimensional Spin-manifold, and $P_{S O(n)}$ denote the principal frame bundle over $X$. Let A be the lift of the Levi-Civita connection to $Q_{\operatorname{Spin}(n)} \longrightarrow$ $X$ and $u \in C^{\infty}\left(Q_{\operatorname{Spin}(n)}, \mathrm{W}\right)^{\operatorname{Spin}(n)}$. Denote by $D_{\mathrm{A}}$ the covariant derivative given by

$$
D_{\mathrm{A}} u=T u+\mathrm{A} \cdot u \in C^{\infty}\left(Q_{\operatorname{Spin}(n)}, \mathrm{W}\right)^{\operatorname{Spin}(n)} .
$$

Composing this with the Clifford multiplication, we obtain the Dirac operator

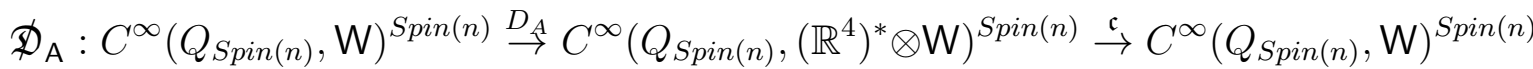

Example 2.5.5. Consider again the case for dimension four. Since in dimension four, the Clifford multiplication by any $v \in \mathbb{R}^{4} \cong\left(\mathbb{R}^{4}\right)^{*}$ interchanges the positive and negative spinor representations, the Dirac operator

$$
\left(\begin{array}{cc}
0 & \not{D}_{\mathrm{A}}^{-} \\
\mathfrak{P}_{\mathrm{A}}^{+} & 0
\end{array}\right): C^{\infty}\left(Q_{\operatorname{Spin}(4)}, \mathbb{W}\right)^{\operatorname{Spin}(4)} \longrightarrow C^{\infty}\left(Q_{\operatorname{Spin}(4)}, \mathbb{W}\right)^{\operatorname{Spin}(4)}
$$

The Dirac operator is a first order, elliptic partial differential operator and is formally self adjoint ([Nic96] Prop. 10.1.41)

\subsection{6 $\operatorname{Spin}^{c}(n)$-structures}

The entire exposition given above for Clifford algebras and Spin-structures can be extended to complexified Clifford algebras

$$
\mathbb{C} l_{n}=\mathcal{C} l_{n} \otimes \mathbb{C}
$$

and $\operatorname{Spin}^{c}$-structures. In this case one obtains the exact sequence:

$$
0 \longrightarrow \mathbb{Z}_{2} \longrightarrow \operatorname{Spin}(n) \times_{ \pm 1} S^{1} \stackrel{\tilde{\lambda}}{\longrightarrow} S O(n) \times S^{1} \longrightarrow 0,
$$

where the map $\tilde{\lambda}: \operatorname{Spin}(n) \times_{ \pm 1} S^{1} \longrightarrow S O(n) \times S^{1}$ is given by

$$
\tilde{\lambda}([a, z])=\left(\lambda(a), z^{2}\right) .
$$


We denote the group $\operatorname{Spin}^{c}(n):=\operatorname{Spin}(n) \times_{ \pm 1} S^{1}$.

A $\operatorname{Spin}^{c}(n)$-structure on an n-dimensional Riemann manifold $X$ is a principal $S^{1}$-bundle $P_{S^{1}}$ together with a principal $\operatorname{Spin}^{c}(n)$-bundle $Q_{\operatorname{Spin}^{c}(n)}$ over $X$, which an equivariant double cover

$$
Q_{\operatorname{Spin}^{c}(n)} \longrightarrow P_{S O(n)} \times_{X} P_{S^{1}}
$$

Proposition 2.5.4 ([LM89]). Every almost complex manifold carries a canonical $\operatorname{Spin}^{c}(n)$ structure.

Note also that every Spin manifold carries a $S p i n^{c}$-structure

A representation of the complexified Clifford algebra induces a complex representation of $\operatorname{Spin}(n) \subset \mathbb{C} l_{n}$, which we shall denote by $\mathrm{W}_{\mathbb{C}}$.

Lemma 2.5.6 ([Mor96] Cor. 2.6.3). A complex representation of the group $\kappa: \operatorname{Spin}(n) \longrightarrow \mathbf{W}_{\mathbb{C}}$ extends uniquely to a representation $\tilde{\kappa}: \operatorname{Spin}^{c}(n) \longrightarrow \mathbf{W}_{\mathbb{C}}$.

Example 2.5.6. Consider the case $n=4$. As a consequence of Lemma (2.5.6), the irreducible complex representations of $\mathbb{W}^{+}$and $\mathbb{W}^{-}$extend to a representations $\mathbb{S}^{+}$and $\mathbb{S}^{-}$, of $\operatorname{Spin}^{c}(4)$. Explicitly, $\mathbb{S}^{+}$is the representation of $\operatorname{Spin}^{c}(4)$ on $\mathbb{H}$ by

$$
\left[q_{+}, q_{-}, z\right] \cdot h \mapsto q_{+} h z
$$

and $\mathbb{S}^{+}$is the representation of $\operatorname{Spin}^{c}(4)$ on $\mathbb{H}$ by

$$
\left[q_{+}, q_{-}, z\right] \cdot h \mapsto q_{-} h z
$$

A connection $A$ on the principal $S^{1}$-bundle coupled with the lift of Levi-Civita connection determine a unique connection $\hat{A}$ on the principal $\operatorname{Spin}^{c}(n)$-bundle. The covariant derivative is defined in an analogous manner to the $\operatorname{Spin}(n)$-case. Composed with the Clifford multiplication, this defines a twisted Dirac operator

$$
\oiint_{\hat{\mathrm{A}}}: C^{\infty}\left(Q_{\operatorname{Spin}^{c}(n)}, \mathbb{S}\right) \longrightarrow C^{\infty}\left(Q_{\operatorname{Spin}^{c}(n)}, \mathbb{S}\right)
$$




\title{
3. HyperKähler manifolds
}

\author{
It is a capital mistake to theorize before one has data. Insensibly, one begins to twist \\ facts to suit theories, instead of theories to suit facts! \\ - Sir Aurthur Conan Doyle, A Study in Scarlet
}

In this chapter we discuss hyperKähler manifolds and focus our attention on the properties on those which admit a permuting $S p(1)$-action and give a brief summary of results on the same.

\subsection{Preliminaries}

An almost complex structure on a manifold $M$ is an endomorphism $I \in \operatorname{End}(T M)$ such that $I^{2}=-1$. If $M$ admits two anti-commuting almost complex structures $I, J \in \operatorname{End}(T M)$, we can define the third one $K=I J$. The triple $\{I, J, K\}$ satisfies quaternionic relations $I^{2}=J^{2}=K^{2}=I J K=-1$. There exists a unique torsion-free affine connection $A$ (Obata connection) on $M$ such that $\nabla^{A} I=\nabla^{A} J=$ $\nabla^{A} K=0$ [Oba58]. That is the almost complex structures are integrable.

A manifold $M$ is said to possess a hypercomplex structure if it admits a pair of anti-commuting complex structures. The existence of a hypercomplex structure implies that the real dimension of the manifold $M$ is $4 n$ and the holonomy is reduced to a subgroup of $G L(n, \mathbb{H})$.

A hypercomplex structure on $M$ induces an algebra homomorphism:

$$
\begin{gathered}
\mathrm{I}: \mathbb{H} \longrightarrow \operatorname{End}(T M) \\
h \longmapsto h_{0} I d_{T M}+h_{1} I_{1}+h_{2} I_{2}+h_{3} I_{3}:=\mathrm{I}_{h} .
\end{gathered}
$$

Let $S p(1)$ denote the group of unit quaternions and $\mathfrak{s p}(1)$ be its Lie algebra. Identifying $\mathfrak{s p}(1)$ with $\mathfrak{I m}(\mathbb{H})$, the imaginary quaternions, the restriction of I to 
$\mathfrak{I m}(\mathbb{H})$ gives a map

$$
\begin{gathered}
\mathfrak{s p}(1) \longrightarrow \operatorname{End}(T M) \\
\eta \longmapsto \mathrm{I}_{\eta} .
\end{gathered}
$$

For any $\eta \in \mathfrak{s p}(1)$, we have $\nabla^{A} \mathrm{I}_{\eta}=0$. If $\eta$ is such that $\eta^{2}=-1$, then $\mathrm{I}_{\eta}^{2}=\mathrm{I}_{\eta^{2}}=$ $-I d_{T M}$ and $\mathrm{I}_{\eta}$ is again a complex structure. Observe that $M$ has an entire family of complex structures parametrized by $S^{2} \in \mathfrak{I m}(\mathbb{H})$.

\subsubsection{HyperKähler Manifolds}

Let $\left(M, g_{M}\right)$ be a Riemann manifold endowed with a hypercomplex structure. If for all $\eta \in \mathfrak{s p}(1)$, the metric satisfies $g^{M}\left(\mathrm{I}_{\eta} X, \mathrm{I}_{\eta} Y\right)=|\eta|^{2} g^{M}(X, Y)$ then it is said to be compatible with the hypercomplex structure.

Definition 3.1.1. (HyperKähler manifold) Let $M$ be a hypercomplex manifold with a compatible metric. If the Obata connection coincides with the Levi-Civita connection, then the manifold is called as HyperKähler manifold.

Alternatively, a hyperKähler manifold can be charaterized as follows: Define a two form $\omega \in \mathfrak{s p}(1)^{*} \otimes \Omega^{2}(M)$ by:

$\omega(\eta)(X, Y):=\omega_{\eta}(X, Y)=g_{M}\left(\mathrm{I}_{\eta} X, Y\right)$ for $\eta \in \mathfrak{I m}(\mathbb{H}) \cong \mathfrak{s p}(1), \quad X, Y \in \Gamma(M, T M)$

Proposition 3.1.1. [Hit87] Given a Riemann manifold $M$ endowed with complex structures $\left(I_{1}, I_{2}, I_{3}\right)$ satisfying the quaternionic relations, a sufficient condition for $M$ to be a hyperKähler manifold is that the two forms $\left\{\omega_{l}\right\}_{l=1}^{3}$ defined by

$$
\omega_{l}(X, Y)=g_{M}\left(I_{l} X, Y\right)
$$

for $l=1,2,3$, are closed.

Note 3.1.1. This is in contrast to the Kähler case where, for an almost complex structure $I$, the condition $d \omega_{I}=0$ does not guarantee that $I$ is integrable.

The 2-forms $\omega_{l}$ being non-degenerate, the above conditions are equivalent to the requirent that $\omega_{l}$ be a symplectic form for each $I_{l}$. 
Remark 3.1.1. Clubbing the above 3 conditions into one we get:

$$
\omega=\omega_{1} i+\omega_{2} j+\omega_{3} k \in \mathfrak{s p}(1) \otimes \Omega^{2}(M) .
$$

Then the equivalent condition for $M$ to be hyperKähler is $d \omega=0$.

Example 3.1.1. An example of a hyperKähler manifold is the flat space $\mathbb{H}^{n}$. The scalar multiplication is given by $\mathrm{I}_{h}(X)=X \cdot \bar{h}$ for $x \in \mathbb{H}^{n}, \quad X \in T_{x} \mathbb{H}^{n} \cong \mathbb{H}^{n}, \quad h \in$ $\mathbb{H}$.

Example 3.1.2. Another source of hyperKähler manifolds is via the hyperKähler reduction. Given a (locally)free action of a compact Lie group $G$ on a manifold $M$, preserving the symplectic two form $\omega$, we can define a moment map $\mu: M \longrightarrow g^{*}$ for the $G$-action (ref. Section 3.2.3 ). Let $\mathfrak{g}$ be the Lie algebra of $G$ and choose a point $b \in \mathfrak{g}^{*}$. Let $G^{\prime}$ be the isotropy group at a point. Then the quotient $\mu^{-1}\{b\} / G^{\prime}$ inherits the symplectic structure. This is called the Marsden-Weinstein reduction. This was generalized to hyperKähler manifolds by Hitchin et al. [HKLR87].

\subsection{Properties of hyperKähler manifolds with per- muting action}

\subsubsection{Permuting action}

Definition 3.2.1. An action of $S p(1)$ on a hyperKähler manifold $M$ is said to be permuting if $S p(1)$ acts by isometries and the induced action on the sphere of complex structures is given by $T q \mathrm{I}_{\eta} T q^{-1}=\mathrm{I}_{q \eta \bar{q}}$ for $q \in S p(1)$ and $\eta \in$ $\mathfrak{s p}(1), \quad\|\eta\|^{2}=1$.

Example 3.2.1. Consider once again, the flat space $\mathbb{H}^{n}$. Define the $S p(1)$ action on $\mathbb{H}^{n}$ by $q \cdot h=h \bar{q}$, for $q \in S p(1)$ and $h \in \mathbb{H}^{n}$. The tangent bundle on $\mathbb{H}^{n}$ is trivial and the complex structures are given by:

$$
I_{1}(v)=v \bar{i}, \quad I_{2}(v)=v \bar{j}, \quad I_{3}(v)=v \bar{k} \text { for } x \in \mathbb{H}^{n} \text { and } v \in T_{x} \mathbb{H}^{n} \text {. }
$$

The induced action of $S p(1)$ on the tangent bundle is again given by $q_{*} v=v \bar{q}$. Let $x \in \mathbb{H}^{n}$ and $v \in T_{x} \mathbb{H}^{n}$. Let $\eta \in \mathfrak{s p}(1)$ with $\|\eta\|^{2}=1$ and $\mathrm{I}_{\eta}$ be the corresponding 
complex structure. Then the induced action on the sphere of complex structures is given by

$$
q \cdot \mathrm{I}_{\eta}=\bar{q} \mathrm{I}_{\eta} q, \quad \bar{q} \mathrm{I}_{\eta} q(v)=v \bar{q} \bar{\eta} q=v(\overline{q \eta \bar{q}})=\mathrm{I}_{\operatorname{Ad}_{q}(\eta)}(v) .
$$

Thus we see that the action is permuting.

HyperKähler manifolds with permuting actions were first studied by Swann [Swa91].

In what follows, we shall restrict ourselves to hyperKähler manifolds with permuting action of $S p(1)$. We closely follow the exposition given in [BGM93], [Pid04] and [Sch10].

Define the map $K^{M}: \mathfrak{s p}(1) \longrightarrow \Gamma(M, T M)$ given by $K^{M}(\xi)=K_{\xi}^{M}$, the fundamental vector field generated by $\xi$. Let $\zeta \in S p(1)$. Then,

$$
K^{M}(\zeta \cdot \xi)=K_{A d_{\zeta} \xi}^{M}=T \zeta\left(K_{\xi}^{M}\right)=\zeta \cdot K_{\xi}^{M}
$$

Thus $K^{M}$ is an $S p(1)$-equivariant map. Similarly, the map I restricted to $\mathfrak{I m}(\mathbb{H}) \cong$ $\mathfrak{s p}(1)$ is also an $S p(1)$-equivariant map.

Both the maps being linear, we combine them into one $S p(1)$-equivariant map:

$$
\begin{gathered}
\mathcal{X} \in \mathfrak{s p}(1)^{*} \otimes \mathfrak{s p}(1)^{*} \otimes \Gamma(M, T M) \\
\mathcal{X}(\xi \otimes \zeta)=\mathrm{I}(\xi) K^{M}(\zeta)=\mathrm{I}_{\xi} K_{\zeta}^{M} .
\end{gathered}
$$

Let $W$ denote the standard representation of the group $S p(1)$ on $\mathbb{H}$. We know that $\mathfrak{s p}(1) \cong \mathfrak{s u}(2)$ and therefore $\mathfrak{s p}(1) \otimes \mathbb{C} \cong \mathfrak{s u}(2) \otimes \mathbb{C}=\mathfrak{s l}(2, \mathbb{C})$.

It is well-known that any finite dimensional irreducible representation of $\mathfrak{s l}(2, \mathbb{C})$ is a symmetric power of the standard representation $\mathbb{C}^{2}$. Since the group $S p(1)$ preserves the quaternionic structure on $W$, it also preserves the symmetric powers of the quaternionic structure. The even symmetric powers of the quaternionic structure is a real structure. So the even symmetric powers of the representation admit a real structure, which is preserved by $S p(1)$, and therefore descends to a representation of the real form $\mathfrak{s u}(2) \cong \mathfrak{s p}(1)$. Therefore by Clebsch-Gordon theorem:

$$
\mathfrak{s p}(1)^{*} \otimes \mathfrak{s p}(1)^{*} \cong \mathbb{R} \oplus\left[S^{2}(W)\right]_{r} \oplus\left[S^{4}(W)\right]_{r} .
$$


On the other hand, $\mathfrak{s p}(1)^{*} \otimes \mathfrak{s p}(1)^{*}$ splits into a direct sum of sub-representations $S^{2}\left(\mathfrak{s p}(1)^{*}\right) \oplus \wedge^{2}\left(\mathfrak{s p}(1)^{*}\right)$. The symmetric part further decomposes as $S^{2}\left(\mathfrak{s p}(1)^{*}\right)=$ $\mathbb{R} \oplus S_{0}\left(\mathfrak{s p}(1)^{*}\right)$, corresponding to the trace and the traceless part, respectively.

Therefore $\mathfrak{s p}(1)^{*} \otimes \mathfrak{s p}(1)^{*}=\mathbb{R} \oplus S_{0}\left(\mathfrak{s p}(1)^{*}\right) \oplus \wedge^{2} \mathfrak{s p}(1)^{*} \cong \mathbb{R} \oplus\left[S^{4}(W)\right]_{r} \oplus\left[S^{2}(W)\right]_{r}$. Corresponding to this decomposition, the map $\mathcal{X}$ splits into three parts: $\mathcal{X}_{0}, \mathcal{X}_{2}$, $\mathcal{X}_{1}$ respectively. Denote by $A l t$, the projection of $\mathfrak{s p}(1)^{*} \otimes \mathfrak{s p}(1)^{*}$ to the alternating part $\Lambda^{2} \mathfrak{s p}(1)^{*}$ and by $S y m$, the projection of $\mathfrak{s p}(1)^{*} \otimes \mathfrak{s p}(1)^{*}$ to the symmetric part $S^{2}\left(\mathfrak{s p}(1)^{*}\right)$.

We have the isomorphism $[\cdot, \cdot]: \Lambda^{2} \mathfrak{s p}(1) \longrightarrow \mathfrak{s p}(1)$ given by

$$
\begin{aligned}
& i \wedge j \longmapsto[i, j]=2 k \\
& j \wedge k \longmapsto[j, k]=2 i \\
& k \wedge i \longmapsto[k, i]=2 j .
\end{aligned}
$$

The dual of this map is $[\cdot, \cdot]^{*}: \mathfrak{s p}(1)^{*} \longrightarrow \Lambda^{2} \mathfrak{s p}(1)^{*}$.

Denote by $\pi_{1}^{*}$ the map $\pi_{1}^{*}=\mathfrak{s p}(1)^{*} \otimes \mathfrak{s p}(1)^{*} \stackrel{A l t}{\longrightarrow} \Lambda^{2} \mathfrak{s p}(1)^{*} \stackrel{\left(-[, \cdot]^{*}\right)^{-1}}{\longrightarrow} \mathfrak{s p}(1)^{*}$. Then:

$$
\begin{aligned}
\mathcal{X}_{0} & =-\frac{1}{3} \operatorname{tr} \mathcal{X} & & \in & \Gamma(M, T M) \\
\mathcal{X}_{1}=\pi_{1}^{*}(\mathcal{X}) & & \in & & \mathfrak{s p}(1)^{*} \otimes \Gamma(M, T M) \\
\mathcal{X}_{2}=-\mathcal{X}_{0}\langle\cdot, \cdot\rangle_{\mathbb{H}}-\operatorname{Sym} \mathcal{X} & & \in & & S_{0}^{2}\left(\mathfrak{s p}(1)^{*}\right) \otimes \Gamma(M, T M)
\end{aligned}
$$

Define the following operators:

$$
\begin{aligned}
\iota_{\mathfrak{s p}(1)}: \otimes^{p} \mathfrak{s p}(1)^{*} \otimes \Omega^{q}(M) \underset{ }{\longrightarrow} \mathfrak{s p}(1)^{*} \otimes\left(\mathfrak{s p}(1)^{*}\right)^{\otimes^{p}} \otimes \Omega^{q-1}(M) \text { defined by } \\
\iota_{\mathfrak{s p}(1)}(\alpha)(\xi)=\iota_{K_{\xi}^{M}} \alpha
\end{aligned}
$$

and

$$
\begin{gathered}
\mathcal{L}_{\mathfrak{s p}(1)}: \otimes^{p} \mathfrak{s p}(1)^{*} \otimes \Omega^{q}(M) \longrightarrow \mathfrak{s p}(1)^{*} \otimes\left(\mathfrak{s p}(1)^{*}\right)^{\otimes^{p}} \otimes \Omega^{q}(M) \text { defined by } \\
\mathcal{L}_{\mathfrak{s p}(1)}(\alpha)(\xi)=\mathcal{L}_{K_{\xi}^{M}} \alpha .
\end{gathered}
$$

where $K_{\xi}^{M}$ is the fundamental vector field on $M$ generated by $\xi \in \mathfrak{s p}(1)$. One can verify the Cartan's formula $\mathcal{L}_{\mathfrak{s p}(1)}=d_{\iota_{\mathfrak{s p}(1)}}+\iota_{\mathfrak{s p}(1)} d$.

Lemma 3.2.1. [Pid04] For the two form $\omega$, as defined in (3.1.1), have the following identity:

$$
\mathcal{L}_{\mathfrak{s p}(1)} \omega=-2 \omega .
$$


Proof : We first verify that $\omega$ is $S p(1)$-equivariant. Let $q \in S p(1)$ and $\xi \in$ $\mathfrak{s p}(1)$. Then for the vector fields $V, W$ on $M$

$$
\begin{aligned}
\left\langle q^{*} \omega, \xi\right\rangle(V, W) & =g_{M}\left(I_{\xi}\left(q_{*} V\right), q_{*} W\right)=g_{M}\left(q_{*}^{-1} I_{\xi}\left(q_{*} V\right), W\right) \\
& =g_{M}\left(I_{A d_{q^{-1}}(\xi)}(V), W\right)=\left\langle\omega, A d_{q^{-1}}(\xi)\right\rangle(V, W)
\end{aligned}
$$

For $\xi_{1}, \xi_{2} \in \mathfrak{s p}(1)$, using the identity above, we get:

$$
\begin{aligned}
\left\langle\mathcal{L}_{\mathfrak{s p}(1)} \omega, \xi_{1} \otimes \xi_{2}\right\rangle & =\mathcal{L}_{K_{\xi_{1}}^{M} \omega} \omega\left(\xi_{2}\right)=\left.\frac{d}{d t}\left(L_{\exp \left(-t \xi_{1}\right)}\right)^{*} \omega\left(\xi_{2}\right)\right|_{t=0} \\
& =\left.\frac{d}{d t} \omega\left(A d_{\exp \left(-t \xi_{1}\right)}\left(\xi_{2}\right)\right)\right|_{t=0}=\left\langle\omega,\left.\frac{d}{d t} A d_{\exp \left(-t \xi_{1}\right)}\left(\xi_{2}\right)\right|_{t=0}\right\rangle=-\left\langle\omega,\left[\xi_{1}, \xi_{2}\right]\right\rangle
\end{aligned}
$$

We conclude from isomorphism (3.2.1) that $\mathcal{L}_{\mathfrak{s p}(1)} \omega=-2 \omega$.

Define $\gamma:=-\frac{1}{2} \iota_{\mathfrak{s p}(1)} \omega=-\frac{1}{2} g_{M}(\mathcal{X}, \cdot) \in \mathfrak{s p}(1)^{*} \otimes \mathfrak{s p}(1)^{*} \otimes \Omega^{1}(M)$. Then $d \gamma=\omega$. The map $\gamma$ decomposes into three components:

$$
\begin{array}{rlrl}
\gamma_{0}=-\frac{1}{3} \operatorname{tr}(\gamma) & \in & \Omega^{1}(M) \\
\gamma_{1}=\pi_{1}^{*}(\gamma) & \in & \mathfrak{s p}(1)^{*} \otimes \Omega^{1}(M) \\
\gamma_{2}=\operatorname{Sym}_{0}(\gamma) & & & S_{0} \otimes \Omega^{1}(M)
\end{array}
$$

By construction, it is clear $\gamma$ is $S p(1)$-equivariant. Also notice that right hand side of (3.2.2) lies in $\mathfrak{s p}(1)^{*} \otimes \Omega^{2}(M) \cong \Lambda^{2} \mathfrak{s p}(1)^{*} \otimes \Omega^{2}(M)$ which implies $d \gamma_{1}=\omega$ and $d \gamma_{0}=d \gamma_{2}=0$. But first we need the following lemma.

Lemma 3.2.2. [Pid04] For the operator $\mathcal{L}_{\mathfrak{s p}(1)}$ and the map $\gamma_{1}$ defined above, we have the identity

$$
\mathcal{L}_{\mathfrak{s p}(1)} \gamma_{1}\left(\xi_{1}, \xi_{2}\right)=-\left\langle\gamma_{1},\left[\xi_{1}, \xi_{2}\right]\right\rangle
$$

Proof : We shall first prove that the map $\gamma_{1}$ is $S p(1)$-equivariant. Let $q \in$ $S p(1)$ and $\xi_{1}, \xi_{2} \in \mathfrak{s p}(1)$. Then,

$$
\begin{aligned}
\left(L_{q}\right)^{*} \gamma_{1}\left(\left[\xi_{1}, \xi_{2}\right]\right)(V) & =\gamma_{1}\left(\left[\xi_{1}, \xi_{2}\right]\right)\left(q_{*} V\right) \\
& =g_{M}\left(\left(I_{\xi_{1}} K_{\xi_{2}}^{M}-I_{\xi_{2}} K_{\xi_{1}}^{M}\right), q_{*} V\right) \\
& =g_{M}\left(q_{*}^{-1}\left(I_{\xi_{1}} K_{\xi_{2}}^{M}-I_{\xi_{2}} K_{\xi_{1}}^{M}\right), V\right) \\
& \left.=g_{M}\left(I_{A d_{q^{-1}} \xi_{1}} K_{A d_{q^{-1}} \xi_{2}}^{M}-I_{A d_{q^{-1} \xi_{2}}} K_{A d_{q^{-1} \xi_{1}}}^{M}\right), V\right)
\end{aligned}
$$




$$
=\gamma_{1}\left[A d_{q^{-1}} \xi_{1}, A d_{q^{-1}} \xi_{2}\right](V)=\gamma_{1}\left(A d_{q^{-1}}\left[\xi_{1}, \xi_{2}\right]\right)(V)
$$

Since $\gamma_{1}$ is $S p(1)$-equivariant,

$$
\begin{aligned}
\left\langle\mathcal{L}_{\mathfrak{s p}(1)} \gamma_{1}, \xi_{1} \otimes \xi_{2}\right\rangle & =\mathcal{L}_{K_{\xi_{1}}^{M}} \gamma_{1}\left(\xi_{2}\right)=\left.\frac{d}{d t}\left(L_{\exp \left(-t \xi_{1}\right)}\right)^{*} \gamma_{1}\left(\xi_{2}\right)\right|_{t=0} \\
& =\left.\frac{d}{d t} \gamma_{1}\left(A d_{\exp \left(-t \xi_{1}\right)}\left(\xi_{2}\right)\right)\right|_{t=0}=\left\langle\gamma_{1},\left.\frac{d}{d t} A d_{\exp \left(-t \xi_{1}\right)}\left(\xi_{2}\right)\right|_{t=0}\right\rangle=-\left\langle\gamma_{1},\left[\xi_{1}, \xi_{2}\right]\right\rangle
\end{aligned}
$$

Define the following functions:

$$
\begin{array}{r}
\rho_{0}=\frac{1}{3} \operatorname{tr}\left(\iota_{\mathfrak{s p}(1)} \gamma_{1}\right) \\
\rho_{2}=\operatorname{Sym}_{0}\left(\iota_{\mathfrak{s p}(1)} \gamma_{1}\right)
\end{array}
$$

Then, by definition, $\rho_{0}$ is $S p(1)$-invariant and $\rho_{2}$ is $S p(1)$-equivariant.

Lemma 3.2.3. [Pid04] The one forms $\gamma_{0}$ and $\gamma_{2}$ are exact.

\section{Proof :}

Using the isomorphism $\mathfrak{s p}(1)^{*} \cong \Lambda^{2} \mathfrak{s p}(1)^{*}$, the identity (3.2.6) can be rewritten as $\mathcal{L}_{\mathfrak{s p}(1)} \gamma_{1}=\operatorname{Alt}\left(\iota_{\mathfrak{s p}(1)} \omega\right)$ which implies

$$
d \iota_{\mathfrak{s p}(1)} \gamma_{1}=\operatorname{Alt}\left(\iota_{\mathfrak{s p}(1)} \omega\right)-\iota_{\mathfrak{s p}(1)} d \gamma_{1}=\operatorname{Alt}\left(\iota_{\mathfrak{s p}(1)} \omega\right)-\iota_{\mathfrak{s p}(1)} \omega .
$$

Then from the definitions (3.2.3) and (3.2.5), we conclude that $d \rho_{0}=\gamma_{0}$ and $d \rho_{2}=\gamma_{2}$. Thus the vector fields $\mathcal{X}_{0}$ and $\mathcal{X}_{2}$ are (upto a factor) gradient vector fields of the functions $\rho_{0}$ and $\rho_{2}$ respectively.

\subsubsection{HyperKähler potential}

Given a Kähler manifold $N$ with the complex structure $J$ and Riemann metric $g_{N}$, let $\omega_{N}$ denote the symplectic two-form associated to the complex structure. A real valued function $\rho: N \longrightarrow \mathbb{R}$ is called Kähler potential if it satisfies

$$
-d(J(d \rho))=2 \omega_{N}
$$

In more familiar conventions, $i \partial \bar{\partial} \rho=2 \omega_{N}$. On similar lines, one can define the notion of hyperKähler potential. 
Definition 3.2.2. (HyperKähler potential) Let $\left(M, g_{M}, I_{1}, I_{2}, I_{3}\right)$ be a hyperKähler manifold. Then, a real-valued function $\rho: M \longrightarrow \mathbb{R}$ is called a hyperKähler potential if it is a Kähler potential w.r.t all three complex structures simultaneously, i.e,

$$
-d\left(I_{l}(d \rho)\right)=2 \omega_{l}
$$

for $l=1,2,3$, where $\omega_{l}$ is the symplectic 2 -form associated to the complex structure $I_{l}$.

Observe first that $\iota_{\mathfrak{s p}(1)} \gamma=\rho_{0}+\rho_{2} \in \Omega^{0}\left(M, \mathbb{R} \oplus\left[S^{4}(W)\right]_{r}\right)$ and in case $\rho_{2}=0$, $\rho_{0}$ is a hyperKähler potential (cf. [BGM93], [Pid04]).

Remark 3.2.1. $\rho_{2}=0 \Longrightarrow \mathcal{X}_{2}=0$ since $\mathcal{X}_{2}=\operatorname{grad}\left(\rho_{2}\right)$. On the other hand $\mathcal{X}_{2}=0 \Longrightarrow d \rho_{2}=0$ and therefore $\rho_{2}$ is locally constant. Claim is that $\rho_{2}=0$. For if not, then there exists a $g \in S p(1)$ such that $g \cdot \rho_{2}(x) \neq \rho_{2}(x)$. Since $\rho_{2}$ is $S p(1)$-equivariant, $g \cdot \rho_{2}(x)=\rho_{2}(g x)=\rho_{2}(x)$, a contradiction. Therefore $\rho_{2}=0$.

In the following proposition, the first observation is due to Martin Callies [Cal10], the second and third one are due to Swann [Swa91].

Proposition 3.2.1. Let $M$ be a hyperKähler manifold with a permuting $S p(1)$ action and such that $\mathcal{X}_{2}=0$. Then:

1. $\gamma_{1}=\iota_{\mathcal{X}_{0}} \omega$

2. $\rho_{0}$ is the hyperKähler potential and $g_{M}\left(\mathcal{X}_{0}, \mathcal{X}_{0}\right)=2 \rho_{0}$.

3. $\mathcal{L}_{\mathcal{X}_{0}} \omega=2 \omega$

Note 3.2.1. Even though there may exist may exist more than one hyperKähler potential, there is a specific choice in the case when $\mathcal{X}_{2}=0$, namely $\rho_{0}=$ $\frac{1}{2} g_{M}\left(\mathcal{X}_{0}, \mathcal{X}_{0}\right)$. Henceforth, since we restrict to the case when $\mathcal{X}_{2}=0$, we fix this choice of the hyperKähler potential.

Example 3.2.2. Let $M$ be the flat hyperKähler manifold $\mathbb{H}^{n}$. Identifying $\mathfrak{s p}(1)$ with $\mathfrak{I m}(\mathbb{H})$, the complex structures are given by right multiplication by $\{\bar{i}, \bar{j}, \bar{k}\}$. We have,

$$
\left.\mathcal{X}_{2}\right|_{u}([i, j])=-\left.\mathcal{X}_{0}\right|_{u}\langle i, j\rangle_{\mathbb{H}^{n}}-\frac{1}{2}(u \bar{i} \bar{j}+u \overline{j i})=0
$$




$$
\begin{aligned}
\left.\mathcal{X}_{2}\right|_{u}([j, k]) & =-\left.\mathcal{X}_{0}\right|_{u}\langle j, k\rangle_{\mathbb{H}^{n}}-\frac{1}{2}(u \bar{j} \bar{k}+u \bar{k} \bar{j})=0 \\
\left.\mathcal{X}_{2}\right|_{u}([k, i]) & =-\left.\mathcal{X}_{0}\right|_{u}\langle k, i\rangle_{\mathbb{H}^{n}}-\frac{1}{2}(u \bar{k} \bar{i}+u \bar{i} \bar{k})=0
\end{aligned}
$$

Therefore $\rho_{0}(u)=\frac{1}{2} g_{\mathbb{H}^{n}}(u, u)=\frac{1}{2}\|u\|^{2}$ is the hyperKähler potential.

Theorem 3.2.1 ([Swa91]). Let $N$ be a hyperKähler manifold admitting a permuting $\operatorname{Sp}(1)$ action such that $\mathcal{X}_{0}=-I_{\xi} K_{\xi}^{M},\|\xi\|^{2}=1$ is independent of $\xi \in \mathfrak{s p}(1)$. Then $N$ admits a hyperKähler potential.

\subsubsection{HyperKähler Moment map}

Definition 3.2.3. Let $N$ be a manifold with symplectic form $\omega$. An action of a Lie group $G$ is said to be symplectic, if $G$ preserves the symplectic form $\left(L_{g}^{*} \omega=\omega\right)$. That the $G$-action is symplectic implies that $\iota_{\mathfrak{g}} \omega$ is a closed form. A smooth map $\mu: N \rightarrow \mathfrak{g}^{*}$ is said to be a moment map for the $G$-action is the following conditions are satisfied:

1. $d \mu=\iota_{\mathfrak{g}} \omega$

2. $\mu(g x)=A d_{g}^{*}(\mu(x))$

For a hyperKähler manifold $M$, an action of a Lie group $G$ is said to be $h y$ perKähler action is $G$ acts by isometries and respects the complex structure, i.e $g^{*} I_{l}=I_{l} g^{*}$. We are now is a position to define the notion of a hyperKähler moment map.

Definition 3.2.4. Let $\left(M, g_{M}, I_{1}, I_{2}, I_{3}\right)$ be a hyperKähler manifold with a hyperKähler action of a Lie group $G$. Let $\omega$ denote the one form defined by (3.1.1). A smooth map $\mu: M \longrightarrow \mathfrak{g}^{*} \otimes \mathfrak{s p}(1)^{*}$ is said to be a hyperKähler moment map if it satisfies the following:

1. $d \mu=\iota_{\mathfrak{g}} \omega$

2. $\mu(g x)=A d_{g}^{*}(\mu(x))$ 
The hyperKähler moment map can be written as $\mu=\mu_{1} i+\mu_{2} j+\mu_{3} k$, where $\mu_{1}=\langle\mu, i\rangle, \mu_{2}=\langle\mu, j\rangle$ and $\mu_{3}=\langle\mu, k\rangle$ are the moment maps for the symplectic forms $\omega_{1}, \omega_{1}$ and $\omega_{1}$ respectively. If $M$ admits a permuting action of $S p(1)$, more can be said. The map $\gamma_{1}$ is $G$-invariant, which implies that $\mathcal{L}_{\mathfrak{g}} \gamma_{1}=0$. Also $\mu=-\iota_{\mathfrak{g}} \gamma_{1}$ is a $G$-equivariant map. Therefore,

$$
d \mu=-d \iota_{\mathfrak{g}} \gamma_{1}=-\mathcal{L}_{\mathfrak{g}} \gamma_{1}+\iota_{\mathfrak{g}} \omega
$$

This observation is due to Pidstrygach [Pid04]. Since $\mathcal{X}_{2}=0$, we have $I_{\xi} \mathcal{X}_{0}=K_{\xi}^{M}$. Hence

$$
\gamma_{1}(\xi)=\frac{1}{2} \iota \mathcal{X}_{0} \omega(\xi)=\frac{1}{2} g_{M}\left(K_{\xi}^{M}, \cdot\right)
$$

and this gives an explicit expression for moment map: (c.f [Sch10] )

$$
\mu(\xi \otimes \eta)=-\frac{1}{2} g_{M}\left(K_{\xi}^{M}, K_{\eta}^{M}\right) .
$$

Remark 3.2.2. If $M$ has a hyperKähler action of a compact Lie group $G$, then $\rho_{0}$ is a $S p(1) \times G$-invariant function on $M$ and $\mu$ is $S p(1) \times G$ - equivariant map.

\subsection{Quaternionic Vector Spaces}

Throughout this section, we shall use the identification $\mathfrak{s p}(1) \cong \mathfrak{I m}(\mathbb{H})$. We shall restrict our attention to the special case $M=\mathbb{H}^{n}$, with the quaternionic structure being given by $\mathcal{M}_{h}(X)=X \cdot \bar{h}$ for $x \in \mathbb{H}^{n}$ and $X \in T_{x} \mathbb{H}^{n}$. Denote by $M_{n \times m}$ the space of $\mathbb{H}$-linear maps $L: \mathbb{H}^{m} \longrightarrow \mathbb{H}^{n}$, i.e, $\mathbb{R}$ linear maps $L: \mathbb{H}^{m} \longrightarrow \mathbb{H}^{n}$ that commutes with scalar multiplication. Denote by $L^{\dagger}$ the conjugate transpose of $L$. So for $L_{1}, L_{2} \in M_{n \times m},\left(L_{1} L_{2}\right)^{\dagger}=L_{2}^{\dagger} L_{1}^{\dagger}$.

Let $x \in \mathbb{H}^{n}$. We define a quaternionic hermitian product on $T_{x} \mathbb{H}^{n}$ by

$$
\begin{gathered}
\langle\cdot, \cdot\rangle_{\mathbb{H}}: T_{x} \mathbb{H}^{n} \otimes T_{x} \mathbb{H}^{n} \longrightarrow \mathbb{H} \\
\langle X, Y\rangle_{\mathbb{H}}=X^{\dagger} Y
\end{gathered}
$$

and Riemannian metric $g_{\mathbb{H}}(\cdot, \cdot)=\mathfrak{R e}\left(\langle\cdot, \cdot\rangle_{\mathbb{H}}\right)$. For $X, Y \in T_{x} \mathbb{H}^{n}$ and $\xi \in \mathfrak{s p}(1)$ the hyperKähler 2 -form $\omega$ is given by

$$
\omega(\xi)(X, Y)=g_{\mathbb{H}}\left(I_{\xi} X, Y\right)=\mathfrak{R e}\left((X \cdot \bar{\xi})^{\dagger} Y\right)=\mathfrak{R e}\left(\xi \cdot X^{\dagger} Y\right)
$$


The Lie group $S U(n) \subset S p(n) \subset E n d_{\mathbb{H}}\left(\mathbb{H}^{n}\right)$ acts on $\mathbb{H}^{n}$ by left multiplication $(L, X) \longmapsto L \cdot X$. This action preserves the metric and respects scalar multiplication and hence is hyperKähler. The fundamental vector fields due to this action is given by

$$
\left.K_{\eta}^{M}\right|_{x}=(x, \eta \cdot x) \in T \mathbb{H}^{n}
$$

where $\eta \in S U(n)$.

The Lie group $S p(1)$ acts permuting, the action being given by $(q, x) \longmapsto x \cdot \bar{q}=$ $x \cdot q^{-1}$ and the fundamental vector fields due to this action are given by

$$
\left.K_{\xi}^{M}\right|_{x}=(x,-x \cdot \xi) \in T \mathbb{H}^{n},
$$

where $\xi \in \mathfrak{s p}(1)$.

In this case, $\mathcal{X}_{2}=0$ and hence $\rho_{0}=\frac{1}{2}\|\cdot\|^{2}$ is the hyperKähler potential (See example (3.2.2)).

Let $\mathfrak{s u}(n)$ denote the Lie algebra of $S U(n)$. From (3.2.9), the expression for the hyperKähler moment map $\mu_{S U(n)} \in \mathfrak{s p}(1)^{*} \otimes \mathfrak{s u}(n)^{*} \otimes C^{\infty}\left(\mathbb{H}^{n}, \mathbb{R}\right)$ is easily computed:

$$
\left.\mu_{S U(n)}(\xi \otimes \eta)\right|_{x}=-\frac{1}{2} \mathfrak{R e}\left(\xi x^{\dagger} \eta x\right)
$$

Alternatively, let us take into consideration, the identification $\mathbb{H} \otimes_{\mathbb{C}} \mathbb{C}^{n} \cong \mathbb{H}^{n}$ given by $^{1}$

$$
h \otimes z \longmapsto \overline{h z}
$$

The hyperKähler $S U(n)$-action and the permuting $S p(1)$-action on $\mathbb{H} \otimes_{\mathbb{C}} \mathbb{C}^{n}$ are given by

$$
(A, q, h \otimes z) \longmapsto(q \cdot h) \otimes(\bar{A} \cdot z) .
$$

The moment map $\tilde{\mu}_{S U(n)} \in \mathfrak{s p}(1) \otimes \mathfrak{s u}(n) \otimes C^{\infty}\left(\mathbb{H} \otimes_{\mathbb{C}} \mathbb{C}^{n}, \mathbb{R}\right)$ is given by

$$
\tilde{\mu}_{S U(n)}(h \otimes z)=\frac{1}{4 n} h i \bar{h} \otimes\left(i \bar{z} z^{t}-i\|z\|^{2} \cdot i d_{\mathbb{C}^{n}}\right) .
$$

Let $\eta_{1}, \eta_{2} \in \mathfrak{s u}(n)$. We fix an inner product on $\mathfrak{s u}(n)$ given by

$$
\left\langle\eta_{1}, \eta_{2}\right\rangle_{\mathfrak{s u}(n)}=-2 n \mathfrak{R e}\left(\operatorname{tr}\left(\eta_{1} \eta_{2}\right)\right) .
$$

The Lie algebra $\mathfrak{s p}(1)$ inherits the metric induced by identifying $\mathfrak{s p}(1) \cong \mathfrak{I m}(\mathbb{H})$ and is denoted by $\langle\cdot, \cdot\rangle_{\mathfrak{s p}(1)}$.

\footnotetext{
${ }^{1}$ The complex structure on $\mathbb{H}$ is taken to be right multiplication with $\bar{i}$.
} 
Lemma 3.3.1. Under the isomorphism (3.3.2), the two moment maps defined above are equivalent.

Proof : Let $\eta \in \mathfrak{s u}(n)$. Identify $\mathfrak{s p}(1) \otimes \mathfrak{s u}(n)$ with $\mathfrak{s p}(1)^{*} \otimes \mathfrak{s u}(n)^{*}$ as:

$$
\xi \otimes \eta=\langle\xi, \cdot\rangle_{\mathfrak{s p}(1)} \otimes\langle\eta, \cdot\rangle_{\mathfrak{s u}(n)}
$$

where $\langle\cdot, \cdot\rangle_{\mathfrak{s p}(1)}$ and $\langle\cdot, \cdot\rangle_{\mathfrak{s u}(n)}$ are the inner products on $\mathfrak{s p}(1)$ and $\mathfrak{s u}(n)$ respectively. For $h \in \mathbb{H}, z \in \mathbb{C}^{n}$ and $\eta \in \mathfrak{s u}(n)$ consider the following computations

$$
\begin{aligned}
\left\langle\mu_{S U(n)}(h \otimes z), \eta\right\rangle_{\mathfrak{s u}(n)} & =\frac{1}{4 n} h i \bar{h}\left\langle\left(i \bar{z} z^{t}-i\|z\|^{2} \cdot i d_{\mathbb{C}^{n}}\right), \eta\right\rangle_{\mathfrak{s u}(n)} \\
& =\frac{1}{4 n} h i \bar{h}\left\langle i \bar{z} z^{t}, \eta\right\rangle_{\mathfrak{s u}(n)}-\frac{1}{4 n} h i \bar{h}\left\langle i\|z\|^{2} \cdot i d_{\mathbb{C}^{n}}, \eta\right\rangle_{\mathfrak{s u}(n)} \\
& =\frac{1}{4 n} h i \bar{h}\left(-2 n \mathfrak{R e}\left(\operatorname{tr}\left(i \bar{z} z^{t} \eta\right)-\frac{1}{4 n} h i \bar{h}\left(-2 n \mathfrak{R e}\left(\operatorname { t r } \left(i\|z\|^{2} \cdot i d_{\left.\mathbb{C}^{n} \eta\right)}\right.\right.\right.\right.\right. \\
& =\frac{1}{4 n} h i \bar{h}\left(-2 n \mathfrak{R e}\left(\operatorname{tr}\left(i \bar{z} z^{t} \eta\right)\right.\right. \\
& =-\frac{1}{2} h i \bar{h}\left(\mathfrak{R e}\left(\operatorname{tr}\left(z^{t} \eta i \bar{z}\right)\right)\right)
\end{aligned}
$$

Observe that $\left(z^{t} \eta i \bar{z}\right)^{*}=z^{t} \eta i \bar{z} \Longrightarrow\left(z^{t} \eta i \bar{z}\right)$ is a real number. Therefore

$$
\mathfrak{R e}\left(\operatorname{tr}\left(z^{t} \eta i \bar{z}\right)\right)=z^{t} \eta i \bar{z}
$$

Hence we have:

$$
-\frac{1}{2} h i \bar{h}\left(\mathfrak{R e}\left(\operatorname{tr}\left(z^{t} \eta i \bar{z}\right)\right)\right)=-\frac{1}{2} h i \bar{h}\left(z^{t} \eta i \bar{z}\right)=-\frac{1}{2} h i\left(z^{t} \eta i \bar{z}\right) \bar{h}
$$

Let $x:=\overline{h z}$. Therefore,

$$
-\frac{1}{2} h i\left(z^{t} \eta i \bar{z}\right) \bar{h}=\frac{1}{2} h\left(z^{t} \eta \bar{z}\right) \bar{h}=\frac{1}{2}\left(h z^{t}\right) \eta(\overline{h z})=\frac{1}{2}(h z)^{t} \eta \overline{h z}=\frac{1}{2} x^{*} \eta x \in \mathfrak{s p}(1)
$$

For $\xi \in \mathfrak{s p}(1)$, we have:

$$
\left\langle\xi, \frac{1}{2} x^{*} \eta x\right\rangle_{\mathfrak{s p}(1)}=-\frac{1}{2} \mathfrak{R e}\left(\xi x^{*} \eta x\right)=\left.\tilde{\mu}_{S U(n)}(\xi \otimes \eta)\right|_{x}
$$




\subsection{Swann Bundles over Wolf Spaces.}

In this section we shall briefly outline the construction of Swann bundles. For a more detailed exposition, we refer the interested reader to [Swa91] and [BGM93].

A quaternionic Kähler manifold $N$ is a 4 n-dimensional manifold, $n>1$, whose linear holonomy group lies in $S p(n) S p(1):=S p(n) \times_{\mathbb{Z}_{2}} S p(1) \subset S O(4 n)$. This implies that the manifold is Einstein [Ale68]. Since these are of dimension strictly greater than 2, this implies that their scalar curvature is necessarily constant. If the scalar curvature of $N$ vanishes identically and $N$ is simply connected, then the linear holonomy subgroup is contained in $S p(n)$ and hence $N$ is a hyperKähler manifold. On the other hand, if $N$ is a hyperKähler manifold, then its scalar curvature vanishes and its linear holonomy group is contained in $S p(n) \subset S p(n) S p(1)$.

If $N$ is a symmetric quaternionic Kähler manifold of strictly positive scalar curvature, then it is a homogeneous, compact manifold [Wol65]. Wolf and Alekseevskii [Ale68] gave a classification of compact homogenous quaternionic Kähler manifolds:

$$
\begin{gathered}
\mathbb{H}^{n}=\frac{S p(n+1)}{S p(1) \times S p(n)}, \quad X(n)=\frac{S U(n)}{S(U(n-2) \times U(2))} \\
Y(n)=\frac{S O(n)}{S O(n-4) \times S O(4)}
\end{gathered}
$$

along with 5 exceptional spaces:

$$
\frac{G_{2}}{S O(4)}, \quad \frac{F_{4}}{S p(3) S p(1)}, \quad \frac{E_{6}}{S U(6) S p(1)}, \quad \frac{E_{7}}{S p i n(12) S p(1)}, \quad \frac{E_{8}}{E_{7} S p(1)}
$$

for $n \in \mathbb{N}$. These spaces are also referred to as Wolf spaces.

For defining the notion of a quaternionic Kähler manifold in four dimensions though, a subtlety is involved. Since $S O(4) \cong S p(1) S p(1)$, every 4-dimensional manifold would be quaternionic Kähler by the definition above, but some of the properties of the higher dimensional analogue donot carry over to dimension four with this definition. This is rectified by demanding that in addition to being Einstein, the manifold is also self-dual (A four-dimensional manifold is self-dual if the Weyl curvature tensor is self-dual). 


\subsubsection{Swann Bundles}

Let $F$ denote the $S p(n) S p(1)$ reduction of the principal frame bundle $P_{S O(4 n)}$ of $N$. Then $\mathcal{S}(N)=F / S p(n)$ is a principal $S O(3)$-bundle, which is a frame bundle of the three dimensional vector subbundle of skew symmetric endomorphisms of $T N$. The $S p(1)$ action on $\mathbb{H}$ (by left multiplication) descends to an isometric action of $S O(3)$ on $\mathbb{H}^{*} / \pm 1$. If $N$ has a strictly positive scalar curvature, then the Swann bundle over $N$ is defined to be the principal $\mathbb{H}^{*} / \mathbb{Z}_{2}$-bundle over $N$

$$
\mathcal{U}(N):=\mathcal{S}(N) \times_{S O(3)} \mathbb{H}^{*} / \mathbb{Z}_{2} \longrightarrow N \text {. }
$$

Remark 3.4.1. Given a a quaternionic kähler manifold $N$ with positive scalar curvature, the total space of $\mathcal{U}(N)$ obtains a Riemann metric given by $g_{\mathcal{U}(N)}=$ $g_{\mathbb{H}^{*} / \mathbb{Z}_{2}}+r^{2} g_{N}$ where $r$ is the radial co-ordinate on $\mathbb{H}^{*} / \mathbb{Z}_{2}$ and $g_{\mathbb{H}^{*} / \mathbb{Z}_{2}}$ is the quotient metric obtained from $\mathbb{H}$. One can alternatively write the total space of Swann bundle as $\mathcal{U}(N)=(0, \infty) \times \mathcal{S}(N)$ and its metric as $g_{\mathcal{U}(N)}=d r^{2}+r^{2}\left(g_{N}+g_{\mathbb{R}^{3}}\right)$, where $g_{\mathbb{R}^{3}}$ is the quotient metric on $\mathbb{R} \mathbb{P}^{3}$ obtained from its double cover $S^{3}$. ( $\mathbb{H}^{*} / \mathbb{Z}_{2}$ is a metric cone over $\mathbb{R P}^{3}$ ). This implies that $\mathcal{U}(N)$ is a metric cone over $\mathcal{S}(N)$, with $g_{\mathcal{S}(N)}=g_{N}+g_{\mathbb{R}^{3}}$ and hence, $\mathcal{S}(N)$ is a 3-Sasakian manifold. (A Riemann manifold is 3-sasakian if its metric cone is hyperkähler.)

Theorem 3.4.1 ([Swa91]). Let $N$ be a quaternionic Kähler manifold with a positive scalar curvature. Then $\mathcal{U}(N)$ is a hyperKähler manifold with a permuting $S p(1)$ action and $\mathcal{X}_{2}=0$ holds. Additionally, $\mathcal{U}(N)$ has a hyperKähler potential given by

$$
\rho_{0}=\frac{1}{2} r^{2}
$$

\subsubsection{Orbit Spaces}

Let $G$ be a compact, simply connected, simple Lie group and and let $G^{\mathbb{C}}$ denote its complexification. Analogously, denote by $\mathfrak{g}$ the Lie algebra of $G$ and $\mathfrak{g}^{\mathbb{C}}$ its complexification. Let $\mathfrak{h} \subset \mathfrak{g}^{\mathbb{C}}$ be a Cartan subalgebra and let $\Pi^{+}$be the set of positive roots. Let $\mathfrak{s l}(2, \mathbb{C})$ denote the Lie algebra of $S L(2, \mathbb{C})$. We know that $\mathfrak{s l}(2, \mathbb{C})$ is generated by:

$$
H=\left[\begin{array}{cc}
1 & 0 \\
0 & -1
\end{array}\right], \quad E=\left[\begin{array}{ll}
0 & 1 \\
0 & 0
\end{array}\right], \quad F=\left[\begin{array}{ll}
0 & 0 \\
1 & 0
\end{array}\right]
$$


satisfying

$$
[H, E]=2 E, \quad[H, F]=-2 F, \quad[E, F]=H .
$$

For any $\beta \in \Pi^{+}$, by Jacobson-Morozov theorem ${ }^{2}$, we can find a distinguished subalgebra $\mathfrak{s}_{\beta}$, generated by the triple $\left(H_{\beta}, E_{\beta}, F_{\beta}\right)$, corresponding respectively to the images of $(H, E, F)$ under the Lie algebra embedding

$$
\lambda_{\beta}^{\mathbb{C}}: \mathfrak{s l}(2, \mathbb{C}) \hookrightarrow \mathfrak{g}^{\mathbb{C}}
$$

The real structure on $\mathfrak{s u}(2) \otimes \mathbb{C}$ and $\mathfrak{g} \otimes \mathbb{C}$ commutes with $\lambda_{\beta}^{\mathbb{C}}$ which gives us an embedding of $\mathfrak{s u}(2) \cong \mathfrak{s p}(1)$ in $\mathfrak{g}$. Note that $F_{\beta}=\lambda_{\beta}^{\mathbb{C}}(F)$ is a nilpotent element in $\mathfrak{g}^{\mathbb{C}}$.

Let $\alpha$ denote the highest root. Consider the adjoint action of $G^{\mathbb{C}}$ on $\mathfrak{g}^{\mathbb{C}}$. $F_{\alpha}$ determines the nilpotent orbit $\mathcal{O}_{\alpha}=G^{\mathbb{C}} \cdot F_{\alpha}$. Kronheimer [Kro90] shows that any nilpotent adjoint orbit of the complexification of a compact semisimple and connected Lie group has a natural hyperKähler structure. Futhermore, $\mathcal{O}_{\alpha}$ admits a homothetic $\mathbb{H}^{*}$-action and is a Swann bundle over some homogeneous quaternionic Kähler space, which has to be a homogeneous $G$-space, i.e. a Wolf space [Swa91].

\footnotetext{
${ }^{2}$ Jacobson-Morozov Theorem([Jac51]): Let $\mathfrak{g}$ be a semi-simple Lie algebra and $E$ be a nilpotent element in $\mathfrak{g}$. Then there exist elements $Y, H$, such that the sub-algebra that is generated by $\{E, Y, H\}$ is isomorphic to $\mathfrak{s l}(2, \mathbb{C})$.
} 


\section{Generalized Dirac Operator}

"Stands at the sea... wonders at wondering... I... a universe of atoms... an atom in the universe."

- R. Feynman, "The Value of Science", The Pleasure of Finding Things Out

In this chapter we introduce the non-linear Dirac Operator in dimension four in Section 4.3. We analyze the effect of conformal change of metric on the base manifold in Section 4.4 for a class of hyperKähler manifolds for which $\rho_{2}=0$. We introduce the Fueter operator and quaternionic maps in Section 4.6 and study their relation with the harmonic spinors. An exposition on the same may be found in [Hay08].

\section{1 $\operatorname{Spin}_{\varepsilon}^{G}(n)$-structure}

The first ingredient we need in order to define the non-linear Dirac operator, is a $\operatorname{Spin}_{\varepsilon}^{G}(n)$-structure. This is a generalization of the familiar $\operatorname{Spin}(n)$ and $\operatorname{Spin}^{c}(n)$ structure, where in the latter case, the group $S^{1}$ is now replaced by a compact Lie group $G$. Throughout, we shall assume $n \geq 3$.

\subsubsection{The group $\operatorname{Spin}_{\varepsilon}^{G}(n)$}

Let $G$ be a compact Lie group and $\varepsilon$ be an element of order two in the centralizer $Z(G)$ of $G$. The element $(-1, \varepsilon) \in \operatorname{Spin}(n) \times G$ generates a normal subgroup of order two, denoted by $\langle(-1, \varepsilon)\rangle$. We define $\operatorname{Spin}_{\varepsilon}^{G}(n)=\operatorname{Spin}(n) \times_{ \pm 1} G$. We have the following exact sequence:

$$
0 \longrightarrow\langle(-1, \varepsilon)\rangle \longrightarrow \operatorname{Spin}_{\varepsilon}^{G}(n) \stackrel{\Pi_{G}}{\longrightarrow} S O(n) \times G /\{\varepsilon\} \longrightarrow 0
$$

where $\Pi_{G}$ is the quotient map. 
Example 4.1.1. A familiar case is when $G=S^{1}$ and $\varepsilon=-1$.

Then $\operatorname{Spin}(n) \times_{ \pm 1} S^{1}=\operatorname{Spin}^{c}(n)$.

Since $G$ is a normal subgroup of $\operatorname{Spin}_{\varepsilon}^{G}(n)$, we get the following short-exact sequence:

$$
0 \longrightarrow G \longrightarrow \operatorname{Spin}_{\varepsilon}^{G}(n) \longrightarrow S O(n) \longrightarrow 0
$$

\subsection{2 $\operatorname{Spin}_{\varepsilon}^{G}(n)$ structure}

Let $X$ be a $n$-dimensional Riemann manifold. Let $P_{S O(n)} \longrightarrow X$ denote the principal frame bundle over $X$. Then a $\operatorname{Spin}_{\varepsilon}^{G}(n)$-structure over $X$ is a principal $\operatorname{Spin}_{\varepsilon}^{G}(n)$-bundle, which is an equivariant double cover $Q \stackrel{2: 1}{\longrightarrow} P_{S O(n)} \times_{X} P_{G /\{\varepsilon\}}$, along with principal $G /\{\varepsilon\}$-bundle $P_{G /\{\varepsilon\}} \longrightarrow X$. We denote by

$$
\begin{aligned}
& \pi_{S O(n)}: Q_{S p i n \varepsilon^{G}(n)} \longrightarrow P_{S O(n)} \\
& \pi_{G /\{\varepsilon\}}: Q_{S p i n E_{\varepsilon}^{G}(n)} \longrightarrow P_{G /\{\varepsilon\}}
\end{aligned}
$$

In general, the $P_{G /\{\varepsilon\}}$ does not lift to a principal $G$-bundle $P_{G} \longrightarrow X$. The obstruction can be understood in terms of Čech cohomology class. Let $\left\{\mathcal{U}_{\alpha}\right\}$ denote a cover of $X$ such that $\mathcal{U}_{\alpha} \cap \mathcal{U}_{\beta}$ is contractible. Let $g_{\alpha \beta}: \mathcal{U}_{\alpha} \cap \mathcal{U}_{\beta} \longrightarrow$ $G /\{\varepsilon\}$. Then $\left\{g_{\alpha \beta}\right\}_{\alpha, \beta \in 1}:=\mathrm{g} \in \check{H}^{1}(X, G /\{\varepsilon\})$. Let $g_{\alpha \beta}: \mathcal{U}_{\alpha} \cap \mathcal{U}_{\beta} \longrightarrow G /\{\varepsilon\}$ be any representative of $\mathbf{g}$. Since $\mathcal{U}_{\alpha} \cap \mathcal{U}_{\beta}$ is contractible, $g_{\alpha \beta}$ can be lifted to $\tilde{g}_{\alpha \beta}: \mathcal{U}_{\alpha} \cap \mathcal{U}_{\beta} \longrightarrow G$. By the exactness of the sequence :

$$
0 \longrightarrow \mathbb{Z}_{2} \longrightarrow G \longrightarrow G /\{\varepsilon\} \longrightarrow 0
$$

we get that $\eta_{\alpha \beta \gamma}:=\tilde{g}_{\alpha \beta} \tilde{g}_{\beta \gamma} \tilde{g}_{\beta \gamma}: \mathcal{U}_{\alpha} \cap \mathcal{U}_{\beta} \cap \mathcal{U}_{\gamma} \longrightarrow\{1, \varepsilon\}$. In other words, $\left\{\eta_{\alpha \beta \gamma}\right\}$ is a Čech cocycle and represents a cohomology class $w_{G} \in \check{H}^{2}\left(X, \mathbb{Z}_{2}\right)$. This defines the map $w_{G}: \check{H}^{1}(X, G /\{\varepsilon\}) \longrightarrow \check{H}^{2}\left(X, \mathbb{Z}_{2}\right)$. The lift of $\mathbf{g}$ to $\tilde{\mathbf{g}}$, fulfills the cocycle condition iff

$$
w_{G}\left(P_{G /\{\varepsilon\}}\right)=0(\bmod 2) .
$$

In the $\operatorname{Spin}(n)$-case, $w_{G}$ is known to be the second Steifel-Whitney class $w_{2}$, which is the obstruction to lifting the principal $S O(n)$-bundle to $\operatorname{Spin}(n)$-bundle. 
By the same reasoning, the short exact sequence (4.1.1) implies that a $\operatorname{Spin}_{\varepsilon}^{G}(n)$ structure over $X$ will exist iff $w_{S p i n_{\varepsilon}^{G}(n)}\left(P_{S O(n)} \times_{X} P_{G /\{\varepsilon\}}\right)=0(\bmod 2)$. By naturalness of constructions:

$$
w_{\operatorname{Spin} n^{G}(n)}\left(P_{S O(n)} \times_{X} P_{G /\{\varepsilon\}}\right)=w_{2}\left(P_{S O(n)}\right)+w_{G}\left(P_{G /\{\varepsilon\}}\right) .
$$

\subsection{Generalized Spinors}

Let $X$ be four dimensional manifold and $\left(M, g_{M}, I_{1}, I_{2}, I_{3}\right)$ be a hyperKähler manifold. The action of the group $\operatorname{Spin}_{\varepsilon}^{G}(3)$ on $M$ is said to be permuting if the action of $\operatorname{Spin}(3) \cong S p(1)$ on $M$ is permuting and the action of $G$ is hyperKähler. Denote by $\vartheta_{+}$the homomorphism $\operatorname{Spin}_{\varepsilon}^{G}(4) \longrightarrow \operatorname{Spin}_{\varepsilon}^{G}(3)$ given by $\left[q_{+}, q_{-}, g\right] \longmapsto\left[q_{+}, g\right]$. We say that the action of $\operatorname{Spin}_{\varepsilon}^{G}(4)$ is permuting if the action is induced by a permuting action of $\operatorname{Spin}_{\varepsilon}^{G}(3)$ via the homomorphism $\vartheta_{+}$. Recall that given a hyperKähler structure, it induces a covariantly constant algebra homomorphism:

$$
\begin{gathered}
\mathrm{I}: \mathbb{H} \longrightarrow \operatorname{End}(T M) \\
h \longmapsto h_{0} I d_{T M}+h_{1} I_{1}+h_{2} I_{2}+h_{3} I_{3}:=\mathrm{I}_{h} .
\end{gathered}
$$

We define $\mathrm{W}^{+}$to be the $\operatorname{Spin}_{\varepsilon}^{G}(4)$-equivariant bundle $T M \longrightarrow M$ equipped with an action induced by $\vartheta_{+}$. More precisely, for any $w_{+} \in \mathbf{W}^{+}$, the action is given by:

$$
\left[q_{+}, q_{-}, g\right] \cdot w_{+}=T q_{+} T g w_{+}
$$

Define the $\mathrm{W}^{-}$to be the $\operatorname{Spin}_{\varepsilon}^{G}(4)$-equivariant bundle $T M \longrightarrow M$ equipped with the following action:

$$
\left[q_{+}, q_{-}, g\right] \cdot w_{-}=\mathrm{I}_{q_{-}} \mathrm{I}_{\bar{q}_{+}} T q_{+} T g w_{-} .
$$

One can check that this defines a left action of $\operatorname{Spin}_{\varepsilon}^{G}(4)$.

Let $Q \longrightarrow X$ denote the principal $\operatorname{Spin}_{\varepsilon}^{G}(4)$ bundle over $X$. Then we define the space of generalized spinors to be $\mathscr{S}:=C^{\infty}(Q, M)^{\operatorname{Spin}_{\varepsilon}^{G}(4)}$. Generalized spinors can be interpreted as smooth sections $\Gamma\left(X, Q \times{ }_{\operatorname{Spin}_{\varepsilon}^{G}(4)} M\right)$.

In case of $G=S^{1}$ and $M=\mathbb{H}$, this is nothing but the space of positive spinors $\mathbb{S}^{+}$. 
Proposition 4.2.1. [Cal10] The space of spinors $\mathscr{S}$ is an infinite-dimensional, smooth, Fréchet manifold. The tangent at a point $u \in \mathscr{S}$ is given by

$$
T_{u} \mathscr{S}=C_{c}^{\infty}(Q, T M)_{u}^{\operatorname{Spin}_{\varepsilon}^{G}(4)}:=\left\{w \in C_{c}^{\infty}(Q, T M)^{\operatorname{Spin}_{\varepsilon}^{G}(4)} \mid \pi_{M} \circ w=u\right\} .
$$

If the base manifold $X$ is compact, then the tangent bundle of $\mathscr{S}$ is given by

$$
T \mathscr{S}=C^{\infty}(Q, T M)^{\operatorname{Spin}_{\varepsilon}^{G}(4)} .
$$

\subsection{Non-linear Dirac Operator}

\subsubsection{Clifford Multiplication}

Recall that, if $\mathrm{W}$ is a $\mathcal{C} l_{n}$-module, restricting it to $\operatorname{Spin}(n)$, Clifford multiplication is a map of $\operatorname{Spin}(n)$-representations:

$$
\mathfrak{m}: \mathbb{R}^{n} \longrightarrow \operatorname{End}(\mathrm{W}) \text {. }
$$

Henceforth, we shall restrict ourselves to the case $n=4$.

In order to define the generalized Dirac operator, we need to define the appropriate Clifford multiplication. The isomorphism $\operatorname{Spin}(4) \cong S p(1)_{+} \times S p(1)_{-}$induces the isomorphism

$$
\operatorname{Spin}_{\varepsilon}^{G}(4) \cong\left(\operatorname{Sp}(1)_{+} \times S p(1)_{-}\right) \times_{ \pm 1} G
$$

We identify $\mathbb{R}^{4}$ with $\mathbb{H}$ by mapping the standard, oriented basis $\left(e_{1}, e_{2}, e_{3}, e_{4}\right)$ of $\mathbb{R}^{4}$, to $(1, \bar{i}, \bar{j}, \bar{k})$. The hyperKähler structure on $\mathbb{H}$ is given by $\left(R_{\bar{i}}, R_{\bar{j}}, R_{\bar{k}}\right)$ and the $\operatorname{Spin}_{\varepsilon}^{G}(4)$ action on $\mathbb{H}$ by $\left[q_{+}, q_{-}, g\right] \cdot h=q_{-} h \bar{q}_{+}$. Define the map

$$
\begin{aligned}
\mathfrak{m}: \mathbb{R}^{4} \cong \mathbb{H} & \longrightarrow \operatorname{End}\left(\mathbf{W}^{+} \oplus \mathbf{W}^{-}\right) \\
h & \longmapsto\left[\begin{array}{cc}
0 & -I_{\bar{h}} \\
I_{h} & 0
\end{array}\right]
\end{aligned}
$$

Since $\mathfrak{m}(h)^{2}=-g_{\mathbb{R}^{4}}(h, h) \cdot i d_{\mathbf{W}^{+} \oplus \mathbf{W}^{-}}$, by universality property, extends to a map of algebras $\tilde{\mathfrak{m}}: \mathcal{C} l_{4} \longrightarrow \operatorname{End}\left(\mathbf{W}^{+} \oplus \mathbf{W}^{-}\right)$. Identifying $\mathbb{R}^{4}$ with $\left(\mathbb{R}^{4}\right)^{*}$ we define Clifford multiplication by:

$$
\begin{gathered}
\mathfrak{c}:\left(\mathbb{R}^{4}\right)^{*} \otimes\left(\mathbf{W}^{+} \oplus \mathbf{W}^{-}\right) \longrightarrow \mathbf{W}^{+} \oplus \mathbf{W}^{-} \\
g_{\mathbb{R}^{4}}(h, \cdot)\left(w_{+}, w_{-}\right) \longmapsto \mathfrak{m}(h)\left(w_{+}, w_{-}\right) .
\end{gathered}
$$

This map is $\operatorname{Spin}_{\varepsilon}^{G}(4)$-equivariant [Sch10]. 


\subsubsection{The Covariant derivative}

Let $\left(X, g_{X}\right)$ be a compact, oriented, Riemann manifold and let $G$ be a compact Lie group. Let $\varepsilon \in Z(G)$ be an element of order two. Denote by $P_{G /\{\varepsilon\}} \longrightarrow X$ principal $G /\{\varepsilon\}$-bundle over $X$. Furthermore assume that $w_{S p i n \varepsilon}^{G(4)}\left(P_{S O(4)} \times_{X} P_{G /\{\varepsilon\}}\right)=0$ and let $Q \stackrel{\pi_{X}}{\longrightarrow} X$ a principal $\operatorname{Spin}_{\varepsilon}^{G}(4)$-bundle over $X$. Let $M$ be a hyperKähler manifold with a permuting action of the group $\operatorname{Spin}_{\varepsilon}^{G}(4)$.

The Levi-Civita connection $\varphi$ on $P_{S O(4)} \stackrel{\pi_{S O(4)}}{\longrightarrow} X$ and a connection B on $P_{G /\{\varepsilon\}^{-}}$ bundle define uniquely a connection $\mathrm{A}:=\pi_{Q}^{*}(\varphi \oplus \mathrm{B})$ on $Q$, where $\pi_{Q}$ is the double cover $Q \stackrel{\pi_{Q}}{\longrightarrow} P_{S O(4)} \times_{X} P_{G /\{\varepsilon\}}$.

Let $\mathscr{C}(Q)$ denote the space of smooth connections on the bundle $Q$ and $\mathscr{A} \subset$ $\mathscr{C}(Q)$ denote the space of all connections which are the lift of the Levi-Civita connection. More precisely, if $\mathfrak{s p i n}_{\varepsilon}^{G}(4)$ denotes the Lie algebra of the group $\operatorname{Spin}_{\varepsilon}^{G}(4)$ and $\mathfrak{g}$ denotes the Lie algebra of the group $G$, then

$$
\mathfrak{s p i n}_{\varepsilon}^{G}(4) \cong \mathfrak{s p i n}(4) \oplus \mathfrak{g} \cong \mathfrak{s o}(4) \oplus \mathfrak{g}
$$

Therefore, $\mathscr{A}=\left\{A \in \mathcal{C}(Q) \mid \operatorname{pr}_{\mathfrak{s o}(4)} \circ A=\pi_{S O(4)}^{*} \varphi\right\}$.

The covariant derivative of a generalized spinor $u \in \mathscr{S}$, w.r.t a connection $\mathrm{A} \in \mathscr{A}$, is given by :

$$
\begin{gathered}
D_{\mathrm{A}}: C^{\infty}(Q, M)^{\operatorname{Spin}_{\varepsilon}^{G}(4)} \longrightarrow C^{\infty}\left(Q,\left(\mathbb{R}^{4}\right)^{*} \otimes T M\right)^{\operatorname{Spin}_{\varepsilon}^{G}(4)} \\
\left\langle D_{\mathrm{A}} u(p), w\right\rangle=\left.T u(\tilde{w})\right|_{p}
\end{gathered}
$$

where, $w \in \mathbb{R}^{4}$, and $\tilde{w}$ denotes the horizontal lift of $\pi_{S O(4)}(p)(w) \in T_{\pi_{X}(p)} X$.

Define the homomorphism of vector bundles

$$
\begin{array}{r}
\left.K_{A}^{M}\right|_{u}: T^{*} Q \longrightarrow u^{*} T M \\
\left.K_{A}^{M}\right|_{u}(v)=\left.K_{A(v)}^{M}\right|_{u(q)} \in T_{u(q)} M
\end{array}
$$

where, $v \in T_{q} Q$ and $K_{\eta}^{M}$ is a fundamental vector field on $M$, corresponding to $\eta \in \mathfrak{s p i n}_{\varepsilon}^{G}(4)$.

Using the map $\left.K_{A}^{M}\right|_{u}$, we can alternatively write the covariant derivative as

$$
D_{\mathrm{A}} u=T u+\left.K_{\mathrm{A}}^{M}\right|_{u} \in C^{\infty}\left(Q,\left(\mathbb{R}^{4}\right)^{*} \otimes u^{*} \mathrm{~W}^{+}\right)^{\operatorname{Spin}_{\varepsilon}^{G}(4)} .
$$


Remark 4.3.1. Let $A_{1}$ and $A_{2}$ be any two connections on $Q$, such that $A_{1}=$ $A_{2}+\alpha$. Then for $v \in T_{q} Q$,

$$
\begin{aligned}
\left.K_{A_{1}}^{M}\right|_{u}(v) & =\left.\frac{d}{d t} \exp t A_{1}(v)\right|_{t=0}=\left.\frac{d}{d t} \exp \left(t A_{2}(v)+t \alpha(v)\right)\right|_{t=0} \\
& =\frac{d}{d t}\left(\left.\exp \left(t A_{2}(v)\right)(\exp (t \alpha(v)))\right|_{t=0}\right. \\
& =\left.K_{A_{2}}^{M}\right|_{u}(v)+\left.K_{\alpha}^{M}\right|_{u}(v)
\end{aligned}
$$

\subsubsection{The Dirac Operator}

Composing Clifford multiplication $\mathfrak{c}$ with the covariant derivative, we get the nonlinear Dirac operator:

$$
\mathscr{D}_{\mathrm{A}} u \in C^{\infty}\left(Q, u^{*} \mathrm{~W}^{-}\right)
$$

The Dirac operator can be better understood as follows:

Define the configuration space $\mathrm{C}=\mathscr{S} \times \mathscr{A}$. Then this a left $\mathscr{G}:=C^{\infty}(Q, G)^{\operatorname{Spin}_{\varepsilon}^{G}(4)_{-}}$ space. The group $\mathscr{G}$ is a normal subgroup of the full gauge group $C^{\infty}\left(Q, \operatorname{Spin}_{\varepsilon}^{G}(4)\right)^{\operatorname{Spin}_{\varepsilon}^{G}(4)}$. Define $\mathbb{W}^{-}=C^{\infty}\left(Q, \mathbf{W}^{-}\right)^{\operatorname{Spin}_{\varepsilon}^{G}(4)}$. Then $\mathbb{W}^{-}$is a $\mathscr{G}$-equivariant vector bundle over $\mathscr{S}$ with the fibre at a point $u \in \mathscr{S}$ being given by $C^{\infty}\left(Q, u^{*} \mathrm{~W}^{-}\right)$[See Prop. 4.2.1]. Hence, for a connection $\mathrm{A} \in \mathscr{A}$ on $Q$, the Dirac operator defines a $\mathscr{G}$-equivariant section of the vector bundle $\mathbb{W}^{-}$.

For more literature on the three and four dimensional non-linear Dirac operator, we refer the interested reader to [Tau99], [Pid04], [Sch10] and [Cal10].

\subsection{Conformal property of the non-linear Dirac operator}

Let $X$ be a four-dimensional, compact Riemann manifold and for a fixed metric $g$ on $X$, let $[g]$ denote the conformal class of $g$. let $f \in C^{\infty}(X, \mathbb{R})$. In the linear case, for two conformally related metrics, $g \& e^{2 f} g$, let $\varphi$ and $\varphi^{\prime}$ be the associated Levi-Civita connections. Then the corresponding Dirac operators are related as ${ }^{1}$

$$
\mathscr{D}_{\varphi^{\prime}}(\mathscr{B} u)=\mathscr{B}\left(e^{-\frac{5}{2} f} \mathscr{D}_{\varphi}\left(e^{\frac{3}{2} f} u\right)\right)
$$

\footnotetext{
1 To the author's knowledge, this formula was first computed by Hitchin [Hit74].
} 
where $u$ is a spinor and the $\mathscr{B}$ denotes an isomorphism of the principal bundles of frames $P_{S O(4)} \stackrel{e^{-f}}{\longrightarrow} P_{S O(4)}$, corresponding to the metrics $g \& e^{2 f} g$ respectively.

\subsubsection{The $C \operatorname{Spin}_{\varepsilon}^{G}(4)$ structure}

The conformal group $C O(n)$ is defined to be $\mathbb{R}^{+} \times S O(n)$. The double cover $\lambda: \operatorname{Spin}(n) \longrightarrow S O(n)$ extends to a double cover

$$
0 \longrightarrow \mathbb{Z}_{2} \longrightarrow \operatorname{CSpin}(n) \stackrel{\widetilde{\Pi}}{\longrightarrow} C O(n) \longrightarrow 0
$$

where, $C \operatorname{Spin}(n):=\mathbb{R}^{+} \times \operatorname{Spin}(n)$. By the same argument, if we define the group $C \operatorname{Spin}_{\varepsilon}^{G}(n):=\mathbb{R}^{+} \times \operatorname{Spin}_{\varepsilon}^{G}(n)$, the double cover 4.1.1 extends to a double cover

$$
0 \longrightarrow \mathbb{Z}_{2} \longrightarrow \operatorname{CSpin}_{\varepsilon}^{G}(n) \stackrel{\widehat{\Pi}_{G}}{\longrightarrow} C O(n) \times G /\{\varepsilon\} \longrightarrow 0
$$

where $\widehat{\Pi}_{G}$ is the quotient map.

Definition 4.4.1. (Conformal frame bundle) A conformal frame bundle over an $n$-dimensional Riemann manifold $N$ is a reduction of the principal frame bundle to the conformal group $C O(n) \subset G L(n)$.

Definition 4.4.2. (Conformal Spin structure) A conformal Spin structure over an $n$-dimensional Riemann manifold $N$ is a principal $C \operatorname{Spin}(n)$-bundle, which is a lift of the conformal frame bundle $P_{C O(n)}$ corresponding to the double cover $\widetilde{\Pi}$ in (4.4.2).

Remark 4.4.1. A conformal spin structure will exist iff a spin-structure exists on the base manifold. A choice of a conformal spin structure is equivalent to choosing a spin-structure on the manifold.

Analogous to the conformal spin structure, we are now in a position to define the conformal $\operatorname{Spin}_{\varepsilon}^{G}(n)$ structure:

Definition 4.4.3. (CSpin $\operatorname{Sp}_{\varepsilon}^{G}(n)$-structure) A $C \operatorname{Spin}_{\varepsilon}^{G}(n)$-structure over an $n$ dimensional Riemann manifold $N$ is a principal $\operatorname{CSpin}_{\varepsilon}^{G}(n)$-bundle, which is a a lift of the principal bundle $P_{C O(n)} \times_{N} P_{G / \varepsilon}$ corresponding to the double cover $\widehat{\Pi}_{G}$ in (4.4.3). 


\subsubsection{Generalized Dirac operator \& Conformal change of metric}

Henceforth, we shall assume that $X$ is a four-dimensional, compact Riemann manifold. Let $P_{C O(4)} \stackrel{\pi_{C O}}{\longrightarrow} X$ be the bundle of conformal frames on $(X,[g])$. A metric on $X$ is a section $g \in \Gamma\left(X, S^{2}\left(T^{*} X\right)\right)$. Fix a metric $g$ on $X$. For $f \in C^{\infty}(X, \mathbb{R})$, consider the metric $g^{\prime}=e^{2 \pi^{*} f} g$ in the conformal class of $g$. The two sections correspond to the reductions $P_{S O(4)} \rightarrow P_{C O(4)}$ and $P_{S O(4)}^{\prime} \rightarrow P_{C O(4)}$ respectively, are given by:

$$
\begin{array}{r}
P_{S O(4)}=\left\{p \in P_{C O(4)} \mid g_{\mathbb{R}^{4}}\left(\theta_{p}, \theta_{p}\right)=\pi^{*} g(\cdot, \cdot)\right\} \\
P_{S O(4)}^{\prime}=\left\{p \in P_{C O(4)} \mid g_{\mathbb{R}^{4}}\left(\theta_{p}, \theta_{p}\right)=\pi^{*}\left(e^{2\left(\pi^{*} f\right)} g\right)(\cdot, \cdot)\right\}
\end{array}
$$

where, $g_{\mathbb{R}^{4}}(\cdot, \cdot)$ is the standard metric on $\mathbb{R}^{4}$ and $\theta$ is the canonical one form on $P_{C O(4)}$ defined by

$$
\theta_{p}(v)=p^{-1}\left(\pi_{C O}(v)\right),
$$

for $p \in P_{C O(4)}$ and $v \in T_{p} P_{C O(4)}$. Note that $\pi^{*} g \in \Gamma\left(P_{C O(4)}, S^{2}\left(\mathbb{R}^{4}\right)^{*}\right)$. Let $\varphi$ be a connection on the principal bundle $P_{C O(4)} \longrightarrow X$ such that:

$$
\left\{\begin{aligned}
(d+\varphi) g & =0 \\
(d+\varphi) \theta & =0
\end{aligned}\right.
$$

Then $\varphi$ is the Levi-Civita connection w.r.t the metric $g$. Let $\varphi^{\prime}$ denote the Levi Civita connection w.r.t the metric $g^{\prime}$ and

$$
\varphi^{\prime}-\varphi=\langle\theta, \xi\rangle \quad \text { where } \quad \xi \in\left(\mathbb{R}^{4}\right)^{*} \otimes \mathfrak{c o}(4)
$$

where $\mathfrak{c o}(4)$ is the lie algebra of $C O(4)=S O(4) \times \mathbb{R}^{+}$. The covariant derivative of the metric $g^{\prime}$ w.r.t $\varphi$ is given by:

$$
\begin{aligned}
(d+\varphi)\left(g^{\prime}\right) & =(d+\varphi) e^{2\left(\pi^{*} f\right)} g \\
& =-e^{2\left(\pi^{*} f\right)} 2\left(\pi^{*} d f\right) g+e^{2\left(\pi^{*} f\right)} d g+e^{2\left(\pi^{*} f\right)} \varphi \cdot g \\
& =-e^{2\left(\pi^{*} f\right)} 2\left(\pi^{*} d f\right) g+e^{2\left(\pi^{*} f\right)}(d+\varphi) g \\
& =-e^{2\left(\pi^{*} f\right)} 2\left(\pi^{*} d f\right) g .
\end{aligned}
$$

Note: The left action of $\operatorname{Aut}\left(\mathbb{R}^{4}\right) \curvearrowright S^{2}\left(\mathbb{R}^{4}\right)^{*}$ is given by

$$
S^{2}\left(\mathbb{R}^{4}\right)^{*} \ni \mathrm{g} \longmapsto b \cdot g(\cdot, \cdot):=g\left(b^{-1}, b^{-1}\right),
$$


where $b \in \operatorname{Aut}\left(\mathbb{R}^{4}\right)$. Therefore the action of an element $\zeta \in \operatorname{Lie}\left(\operatorname{Aut}\left(\mathbb{R}^{4}\right)\right)$ is given by $\zeta \cdot g=-g(\zeta, \cdot)-g(\cdot, \zeta)$. That is the reason we have a negative sign in the second line. If $\zeta \in \mathbb{R}=\operatorname{Lie}\left(\mathbb{R}^{+}\right) \subset \operatorname{Lie}\left(\operatorname{Aut}\left(\mathbb{R}^{4}\right)\right)$, then $\zeta \cdot g=-2 g(\zeta, \cdot)$.

This implies $\left(d+\varphi+\left(\pi^{*} d f\right) \otimes \operatorname{Id}_{\mathbb{R}^{4}}\right)\left(e^{2\left(\pi^{*} f\right)} g\right)=0$. But the torsion of the connection one-form $\varphi+\left(\pi^{*} d f\right) \otimes \operatorname{Id}_{\mathbb{R}^{4}}$ is non-zero, since

$$
\left(d+\varphi+\left(\pi^{*} d f\right) \otimes \operatorname{ld}_{\mathbb{R}^{4}}\right) \theta=\left\langle\pi^{*} d f, \theta\right\rangle
$$

$0 \longrightarrow \mathbb{Z}_{2} \longrightarrow \operatorname{Spin}_{\varepsilon}^{G}(n) \stackrel{\widehat{\Pi}_{G}}{\longrightarrow} C O(n) \times G /\{\varepsilon\} \longrightarrow 0$

To find the expression for $\varphi^{\prime}$, in terms of $\varphi$, such that the torsion of $\varphi^{\prime}$ vanishes, we need to find a one form $\beta \in \Omega^{1}\left(P_{S O(4)}^{\prime}, \mathfrak{s o}(4)\right)^{S O(4)}$ so that

$$
\left(d+\varphi+\left(\pi^{*} d f\right) \otimes \mathbf{I d}_{\mathbb{R}^{4}}+\beta\right) \theta=0 .
$$

Let $T=d \theta+[\varphi, \theta]$ and $T^{\prime}=d \theta+\left[\varphi^{\prime}, \theta\right]$ denote the torsions of the connections $\varphi$ and $\varphi^{\prime}$ respectively. Then

$$
T^{\prime}-T=\left[\varphi^{\prime}-\varphi, \theta\right]
$$

If $D_{p}$ denotes the horizontal subspace at a point $p \in P_{C O(4)}$, the torsion at $p$ is the $\operatorname{map} T(p): \Lambda^{2} D_{p} \cong \Lambda^{2} \mathbb{R}^{4} \stackrel{d \theta}{\longrightarrow} \mathbb{R}^{4}$ and

$$
T^{\prime}(p)_{x y}-T(p)_{x y}=\frac{1}{2}\left(\xi(p)_{x} y-\xi(p)_{y} x\right) \quad x, y \in \mathbb{R}^{4},
$$

where $\xi$ is as in (4.4.5). The first term signifies the result of applying the torsion to $x \wedge y$. In terms of the $G L(4, \mathbb{R})$-equivariant homomorphism:

$\delta:\left(\mathbb{R}^{4}\right)^{*} \otimes \mathfrak{c o}(4) \hookrightarrow\left(\mathbb{R}^{4}\right)^{*} \otimes\left(\mathbb{R}^{4}\right)^{*} \otimes \mathbb{R}^{4} \mapsto \Lambda^{2}\left(\mathbb{R}^{4}\right)^{*} \otimes \mathbb{R}^{4}$, the first map being the inclusion and the second one being the anti-symmeterization, we may write: [Sal89]

$$
T^{\prime}-T=-\delta \xi
$$

For simplicity, we shall use the abbreviation $\Lambda^{k}$ for the space $\Lambda^{k}\left(\mathbb{R}^{n}\right)^{*}$. We know that $\left(\mathbb{R}^{4}\right)^{*} \otimes \mathfrak{c o}(4)=\left(\mathbb{R}^{4}\right)^{*} \otimes \mathfrak{s o}(4) \oplus\left(\mathbb{R}^{4}\right)^{*} \otimes \mathbf{C} \cdot \mathbf{l d}_{\mathbb{R}^{4}}$. There is a natural isomorphism $\mathfrak{s o}(n) \cong \Lambda^{2}\left(\mathbb{R}^{n}\right)^{*}$ obtained by associating to a pair of vectors $v, w \in \mathbb{R}^{n}$, the skewsymmetric endomorphism

$$
v \wedge w=\langle v, \cdot\rangle w-\langle w, \cdot\rangle v
$$


It is easy to see that $\left.\delta\right|_{\mathfrak{s o}(4)}: \Lambda^{1}\left(\mathbb{R}^{4}\right)^{*} \otimes \Lambda^{2}\left(\mathbb{R}^{4}\right)^{*} \mapsto \Lambda^{2}\left(\mathbb{R}^{4}\right)^{*} \otimes \Lambda^{1}\left(\mathbb{R}^{4}\right)^{*}$ is an isomorphism. Therefore

$$
\left.\delta^{-1}\right|_{\mathfrak{s o}(4)}\left(\sum_{j} e^{i} \otimes e_{j} \otimes e^{j}-e^{j} \otimes e_{i} \otimes e^{j}\right)-\left(\sum_{j} e^{j} \otimes e_{j} \otimes e^{i}-e^{j} \otimes e_{i} \otimes e^{j}\right) .
$$

From the computations above for the connections $\varphi$ and $\varphi^{\prime}$, we know that $\xi=\pi^{*} d f$. So,

$$
\operatorname{Tor}\left(\varphi+\pi^{*}(d f)-\left.\delta\right|_{\mathfrak{s o}(4)} ^{-1}\left(\delta\left(\pi^{*} d f\right)\right)\right)-\operatorname{Tor}(\varphi)=-\delta\left(\pi^{*} d f\right)-\delta\left(-\pi^{*} d f\right)=0 .
$$

The map $\pi^{*}(d f) \in C^{\infty}\left(P_{\left.C O(4),\left(\mathbb{R}^{4}\right)^{*}\right)}\right.$. For $p \in P_{C O(4)}$ and a basis element $e_{i} \in$ $\mathbb{R}^{4}$, define $f_{i}(p)=\pi^{*} d f\left(\widetilde{p\left(e_{i}\right)}\right)$, where $\widetilde{p\left(e_{i}\right)}$ is the horizontal lift of $p\left(e_{i}\right)$ with respect to $\varphi$. Thus $f_{i} \in C^{\infty}\left(P_{C O(4)}, \mathbb{R}\right)$. Hence we can write $\pi^{*}(d f)=\sum_{i} f_{i} e^{i} \in$ $C^{\infty}\left(P_{C O(4)},\left(\mathbb{R}^{4}\right)^{*}\right)$. Computations (4.4.7) show that

$$
\left.\delta\right|_{\mathfrak{s o}(4)} ^{-1}\left(\delta\left(\pi^{*} d f\right)\right)=-\sum_{i, j} f_{i}\left(e^{j} \otimes e_{j} \otimes e^{i}-e^{j} \otimes e_{i} \otimes e^{j}\right)
$$

and so

$$
\operatorname{Tor}\left(\varphi+\pi^{*}(d f)+\sum_{i, j} f_{i}\left(e^{j} \otimes e_{j} \otimes e^{i}-e^{j} \otimes e_{i} \otimes e^{j}\right)\right)=0 .
$$

Using the isomorphism (4.4.6):

$$
\varphi^{\prime}=\varphi+\pi^{*}(d f)+\sum_{i, j} f_{i}\left(e^{j} \otimes e_{j} \otimes e^{i}-e^{j} \otimes e_{i} \otimes e^{j}\right)=\varphi+\underbrace{\pi^{*}(d f) \otimes \mathbf{C} \cdot \mathbf{I d}_{\mathbb{R}^{4}}+\sum_{i, j} f_{i} e^{j} \otimes\left(e_{i} \wedge e_{j}\right)}_{\alpha} .
$$

Proposition 4.4.1. ([LM89] Prop. 6.2, Chap. I) The adjoint representation induces the Lie algebra isomorphism $\zeta: \mathfrak{s p i n}(n) \longrightarrow \mathfrak{s o}(n)$ is given by:

$$
\zeta\left(e_{i} e_{j}\right)=2 e_{i} \wedge e_{j},
$$

where, $\left\{e_{i} e_{j}\right\}_{i<j}$ are the basis elements of $\mathfrak{s p i n}(n)$. Consequently for $v, w \in \mathbb{R}^{n}$,

$$
\zeta^{-1}(v \wedge w)=\frac{1}{4}[v, w] .
$$

Therefore for $e_{i} \wedge e_{j} \in \Lambda^{2} \mathbb{R}^{n}, \zeta^{-1}\left(e_{i} \wedge e_{j}\right)=\frac{1}{4}\left(e_{i} e_{j}-e_{j} e_{i}\right)$. Under this isomorphism,

$\pi^{*}(d f) \otimes \mathbf{C} \cdot \mathbf{I d}_{\mathbb{R}^{4}}+\sum_{i, j} f_{i} e^{j} \otimes\left(e_{i} \wedge e_{j}\right) \longmapsto \pi^{*}(d f) \otimes \mathbf{C} \cdot \operatorname{ld}_{\mathbb{R}^{4}}+\frac{1}{4} \sum_{i, j} f_{i} e^{j} \otimes\left(e_{i} e_{j}-e_{j} e_{i}\right)$,

and denoted again by $\alpha$. 
Let $Q$ be the principal $C \operatorname{Spin}_{\varepsilon}^{G}(4)$-bundle over $X$. Recall that for a quaternionic Kähler manifold $N$ of strictly positive scalar curvature, the Swann bundle over $N$ can be written as a Riemann cone $\mathcal{U}(N)=\mathbb{R}^{+} \times \mathcal{S}(N)$, where $\mathcal{S}(N)$ is a 3-Sasakian manifold. Therefore, we have an action of $\mathbb{R}^{+} \times S p(1)$ on $\mathcal{U}(N)=[0, \infty] \times \mathcal{S}(N)$ given by:

$$
\left(\mathbb{R}^{+} \times S p(1)\right) \times \mathbb{R}^{+} \times \mathcal{S}(N) \longrightarrow \mathbb{R}^{+} \times \mathcal{S}(N), \quad((\lambda, q)(r, s)) \longmapsto(\lambda \cdot r, q \cdot s) .
$$

Let $M=\mathcal{U}(N)$ for some quaternionic Kähler manifold $N$ of strictly positive scalar curvature and $u \in C^{\infty}(Q, M)^{\operatorname{Spin}_{\varepsilon}^{G}(4)}$ be a spinor.

Let $\mathrm{A}_{\varphi}$ and $\mathrm{A}_{\varphi^{\prime}}$, be the respective lifts of the Levi-Civita connections $\varphi$ and $\varphi^{\prime}$ to $Q$, for a fixed connection B on $P_{G / \epsilon}$-bundle. We conclude from (4.4.8) and Remark 4.3.1

$$
D_{\mathrm{A}_{\varphi^{\prime}}} u=D_{\mathrm{A}_{\varphi}} u+\left.K_{\alpha}^{M}\right|_{u} \in C^{\infty}\left(Q,\left(\mathbb{R}^{4}\right)^{*} \otimes u^{*} T M\right) .
$$

The target manifold $M$ admits a hyperkähler potential $\rho_{0}$, given by $\rho_{0}(x)=$ $g^{M}\left(\left.\mathcal{X}_{0}\right|_{x},\left.\mathcal{X}_{0}\right|_{x}\right)$. Let $\lambda \in \mathbb{R}^{*}$. Then

$$
\rho_{0}\left(e^{\lambda} x\right)=\frac{1}{2} g^{M}\left(\left.\mathcal{X}_{0}\right|_{e^{\lambda} x},\left.\mathcal{X}_{0}\right|_{e^{\lambda} x}\right)=\frac{1}{2} e^{2 \lambda} g^{M}\left(\left.\mathcal{X}_{0}\right|_{x},\left.\mathcal{X}_{0}\right|_{x}\right)=e^{2 \lambda} \rho_{0}(x) .
$$

So

$$
\left.\frac{d}{d t} \rho_{0}\left(e^{2 t \lambda} x\right)\right|_{t=0}=d \rho_{0}\left(\frac{d}{d t}\left(e^{2 t \lambda} x\right)\right)=\left.2 d \rho_{0}\left(K_{\lambda}^{M, \mathbb{R}^{+}}\right)\right|_{x}=g^{M}\left(\left.\mathcal{X}_{0}\right|_{x},\left.K_{\lambda}^{M, \mathbb{R}^{+}}\right|_{x}\right) .
$$

But

$$
\left.\frac{d}{d t} \rho_{0}\left(e^{2 t \lambda} x\right)\right|_{t=0}=\frac{d}{d t}\left(e^{2 t \lambda}\right) \rho_{0}(x)=2 \lambda \rho_{0}(x)=g_{M}\left(\left.\mathcal{X}_{0}\right|_{x},\left.\mathcal{X}_{0}\right|_{x}\right) .
$$

This gives $\lambda g_{M}\left(\left.\mathcal{X}_{0}\right|_{x},\left.\mathcal{X}_{0}\right|_{x}\right)=\lambda g_{M}\left(\left.\mathcal{X}_{0}\right|_{x},\left.K_{\lambda}^{M, \mathbb{R}^{+}}\right|_{x}\right)$ for every $x \in M$. This implies $K_{\lambda}^{M, \mathbb{R}^{+}}=\lambda \mathcal{X}_{0}$

Theorem 4.4.1. Let $X$ be a four dimensional Riemann manifold and $M$ be the total space of a Swann bundle. Let $f \in C^{\infty}(X, \mathbb{R})$. Then the Dirac operators $\mathscr{D}_{\mathrm{A}_{\varphi}}$ and $\mathscr{D}_{\mathrm{A}_{\varphi^{\prime}}}$, associated to the two conformally related metrics $g \rightsquigarrow g^{\prime}:=e^{2 \pi^{*} f} g$ respectively, acting on a spinor $u \in C^{\infty}\left(P_{C \operatorname{Spin}(4)}, M\right)^{C \operatorname{Spin}(4)}$ are related as:

$$
\mathscr{D}_{\mathrm{A}_{\varphi^{\prime}}}(\mathscr{B} u)=\mathscr{B}\left(T e^{-5 / 2 \pi^{*} f} \mathscr{D}_{\mathrm{A}_{\varphi}}\left(e^{3 / 2 \pi^{*} f} u\right)\right)
$$

where, $\mathscr{B}$ is the lift of the isomorphism $B: P_{C O(4)} \longrightarrow P_{C O(4)}$, given by

$$
p \longmapsto e^{-\pi^{*} f} p \text {. }
$$

Before we give the proof of the theorem, we need the following Lemma. 
Lemma 4.4.1. For $f \in C^{\infty}(X, \mathbb{R})$, we have

$$
\mathscr{D}_{\mathrm{A}}\left(e^{-\pi^{*} f} u\right)=T e^{-\pi^{*} f} \mathscr{D}_{\mathrm{A}} u-\mathfrak{c}\left(d \pi^{*} f\right) \mathcal{X}_{0}
$$

for A, $u$ as in Theorem (4.4.1).

Proof : Let $p \in Q$ and $v \in T_{p} Q$. Let $\gamma:[0,1] \longrightarrow Q$ be a curve in $Q$ such that $\gamma(0)=p$ and $\dot{\gamma}(0)=v$. Evaluating the covariant derivative of $f u$ for $v$ :

$$
D_{\mathrm{A}}\left(e^{-\pi^{*} f} u\right)(v)=T\left(e^{-\pi^{*} f} u\right)(v)+\left.K_{\mathrm{A}(v)}^{M}\right|_{e^{-\pi^{*} f(p)} u(p)}
$$

The first term is given by:

$$
\begin{aligned}
T\left(e^{-\pi^{*} f} u\right)(v) & =\left.\frac{d}{d t}\left(e^{-\pi^{*} f} u\right)(\gamma(t))\right|_{t=0} \\
& =\left.\frac{d}{d t}\left(e^{-\pi^{*} f((\gamma(t))} u(\gamma(t))\right)\right|_{t=0} \\
& =T e^{-\pi^{*} f(p)} T u(v)+K_{\left.\left.T e^{\pi^{*} f} \frac{d}{d t}\left(e^{-\pi^{*} f((\gamma(t))}\right)\right|_{t=0}\right|_{e^{\pi^{*} f} u(p)}} \\
& =T e^{-\pi^{*} f(p)} T u(v)+\left.K_{\left.\left(T e^{\pi^{*} f} T e^{-\pi^{*} f}\right)\left(-d \pi^{*} f((\gamma(0)))\right)\right|_{t=0}}\right|_{e^{\pi^{*} f} u(p)} \\
& =T e^{-\pi^{*} f(p)} T u(v)+\left.K_{\left(-d \pi^{*} f(\gamma(0))\right.}^{M}\right|_{e^{\pi^{*} f} u(p)} \\
& =T e^{-\pi^{*} f(p)} T u(v)+\left(-\left.d \pi^{*} f(\gamma(0)) \mathcal{X}_{0}\right|_{e^{\pi^{*} f} u(p)}\right.
\end{aligned}
$$

Here $T e^{-\pi^{*} f}$ denotes the differential of the action of $e^{-\pi^{*} f}$ on $M$. The second term is

$$
\left.K_{\mathbf{A}(v)}^{M}\right|_{e^{-\pi^{*} f(p)} u(p)}=\left.T e^{-\pi^{*} f(p)} K_{\mathbf{A}(v)}^{M}\right|_{u(p)}
$$

This gives

$$
D_{\mathrm{A}}\left(e^{-\pi^{*} f} u\right)=T e^{-\pi^{*} f} D_{\mathrm{A}} u-\left.d\left(\pi^{*} f\right) \otimes \mathcal{X}_{0}\right|_{e^{-\pi^{*} f} u}
$$

Applying Clifford multiplication, proves the Lemma.

We now give the proof of the Theorem (4.4.1) .

Proof : Let $\mathfrak{c}$ denote the Clifford muliplication w.r.t the metric $g$. Then w.r.t the metric $e^{2 \pi^{*} f} g$ in the conformal class of $g$, the Clifford multiplication is given by $\mathfrak{c}^{\prime}=$ $T e^{-\pi^{*} f} \boldsymbol{c}$. Substituting for $\alpha$ in (4.4.10) and applying the Clifford multiplication we get:

$$
\mathfrak{D}_{\mathrm{A}_{\varphi^{\prime}}} u=T e^{-\pi^{*} f}\left(\mathfrak{P}_{\mathrm{A}_{\varphi}} u+\left.\mathfrak{c}\left(\pi^{*} d f\right) \mathcal{X}_{0}\right|_{u}+\left.\mathfrak{c}\left(K_{\frac{1}{4} \sum_{i, j} f_{i} e^{j} \otimes\left(e_{i} e_{j}-e_{j} e_{i}\right)}^{M, C \operatorname{Spin}(4)}\right)\right|_{u}\right)
$$




$$
\begin{aligned}
& =T e^{-\pi^{*} f}\left(\mathscr{D}_{\mathrm{A}_{\varphi}} u+\left.\mathfrak{c}\left(\pi^{*} d f\right) \mathcal{X}_{0}\right|_{u}+\left.K_{\frac{1}{4} \sum_{i, j} f_{i}\left(e_{j} e_{i} e_{j}-e_{j} e_{j} e_{i}\right)}^{M, C \operatorname{SPin}(4)}\right|_{u}\right) \\
& =T e^{-\pi^{*} f}\left(\mathscr{D}_{\mathrm{A}_{\varphi}} u+\left.\mathfrak{c}\left(\pi^{*} d f\right) \mathcal{X}_{0}\right|_{u}+\left.K_{\frac{1}{4}\left(4 \sum_{i} f_{i} e_{i}-2 \sum_{i, j} f_{i} e_{j} \delta_{i, j}+4 \sum_{i} f_{i} e_{i}\right)}^{M, C \operatorname{Spin}(4)}\right|_{u}\right) \\
& =T e^{-\pi^{*} f}\left(\mathscr{D}_{\mathrm{A}_{\varphi}} u+\left.\mathfrak{c}\left(\pi^{*} d f\right) \mathcal{X}_{0}\right|_{u}+\left.K_{\frac{3}{2} \sum_{i} f_{i} e_{i}}^{M, C \operatorname{SPpin}(4)}\right|_{u}\right) \\
& =T e^{-\pi^{*} f}\left(\mathscr{D}_{\mathrm{A}_{\varphi}} u+\left.\mathfrak{c}\left(\pi^{*} d f\right) \mathcal{X}_{0}\right|_{u}+\left.K_{\frac{3}{2} \pi^{*}(d f)}^{M, C \operatorname{Spin}(4)}\right|_{u}\right) \\
& =T e^{-\pi^{*} f}\left(\mathscr{D}_{\mathrm{A}_{\varphi}} u+\left.\mathfrak{c}\left(\pi^{*} d f\right) \mathcal{X}_{0}\right|_{u}+\left.\frac{3}{2} \pi^{*} d f \cdot \mathcal{X}_{0}\right|_{u}\right)
\end{aligned}
$$

But

$$
\begin{aligned}
\mathscr{D}_{\mathrm{A}_{\varphi^{\prime}}}\left(e^{-\pi^{*} f} u\right) & =T e^{-\pi^{*} f}\left(T e^{-\pi^{*} f} \mathscr{D}_{\mathrm{A}_{\varphi}} u+\left.T e^{-\pi^{*} f} \frac{3}{2} \pi^{*} d f \cdot \mathcal{X}_{0}\right|_{u}\right) \\
& =T e^{-\pi^{*} f}\left(T e^{-\frac{5}{2} \pi^{*} f} \mathscr{D}_{\mathrm{A}_{\varphi}}\left(e^{\frac{3}{2} \pi^{*} f} u\right)\right)
\end{aligned}
$$

This gives

$$
\mathscr{D}_{\mathrm{A}_{\varphi^{\prime}}}(\mathscr{B} u)=\mathscr{B}\left(T e^{-5 / 2 \pi^{*} f} \mathscr{D}_{\mathrm{A}_{\varphi}}\left(e^{3 / 2 \pi^{*} f} u\right)\right) .
$$

\subsection{Weitzenböck Formula}

Let $M=\mathbb{H}^{n+1}$ endowed with a permuting action of $\operatorname{Spin}_{\varepsilon}^{G}(4)$, where $G=S U(n+$ $1)$. Let $\mathrm{B}$ be any fixed connection on the $P_{G /\{\varepsilon\}}$ and let $\mathrm{A} \in \mathscr{A}$ be a connection on $Q$ determined by $\mathrm{B}$. The following is a special case of Weitzenböck formula proved in [Sch10] for $M=\mathbb{H}^{n}$.

Proposition 4.5.1 (Weitzenböck Formula). Let $X$ be a four dimensional, oriented, Riemann manifold. Let $\pi_{Q}: Q \longrightarrow X$ be a principal Spin $\operatorname{Sp}_{\varepsilon}^{G}(4)$ - principal bundle over $X$. Let $\mathrm{A}$ be any connection on $Q$ determined by the Levi-Civita connection on $P_{S O(4)} \longrightarrow X$ and a connection $\mathrm{B}$ on $P_{G / \pm 1} \longrightarrow X$. Denote by $\mathscr{D}_{\mathrm{A}}$ the twisted Dirac operator determined by the connection $\mathrm{A}$. Then for any spinor $u \in C^{\infty}\left(Q, \mathrm{~W}^{+}\right)$, we have:

$$
\mathfrak{D}_{\mathrm{A}}^{*} \mathfrak{D}_{\mathrm{A}} u=D_{\mathrm{A}}^{*} D_{\mathrm{A}} u+\frac{1}{4} s_{X} u+\mathfrak{c}\left(F_{\mathrm{B}}^{+}\right) u .
$$

Corollary 4.5.1. Let $X$ compact, oriented Riemann manifold with a $\operatorname{Spin}_{\varepsilon}^{G}(4)$ structure and let $A$ be the connection as in the above proposition. Let $\mu_{S U(n+1)}$ be 
the moment map for the $S U(n+1)$ action. Then,

$$
\left\|\mathscr{D}_{A} u\right\|_{L^{2}}^{2}=\left\|D_{A} u\right\|_{L^{2}}^{2}+\frac{1}{4} s_{X}\|u\|_{L^{2}}^{2}+2 \int_{X}\left\langle\mu_{S U(n+1)} \circ u, F_{\mathcal{B}}^{+}\right\rangle \operatorname{vol}_{X}
$$

Proof : First observe that an oriented orthonormal basis for $\Lambda_{+}^{2} \mathbb{R}^{4}$ is given by:

$$
\eta_{1}=\frac{1}{2}\left(e_{0} \wedge e_{1}+e_{2} \wedge e_{3}\right), \quad \eta_{2}=\frac{1}{2}\left(e_{0} \wedge e_{2}-e_{1} \wedge e_{3}\right), \quad \eta_{3}=\frac{1}{2}\left(e_{0} \wedge e_{3}+e_{1} \wedge e_{2}\right) .
$$

Identifying $\left(\mathcal{C} l_{4}^{0}\right)_{+}$with $\mathbb{H}$ as in (2.5.2), we have $\mathfrak{c}\left(\eta_{1}\right)=\frac{e_{0} e_{1}+e_{2} e_{3}}{2}=I_{1}$. Similarly, $\mathfrak{c}\left(\eta_{2}\right)=I_{2}$ and $\mathfrak{c}\left(\eta_{3}\right)=I_{3}$. Note that we may write $F_{\mathcal{B}}^{+}=\sum_{l=1}^{3}\left\langle F_{\mathcal{B}}^{+}, \eta_{l}\right\rangle_{\Lambda_{+}^{2}} \eta_{l}$. Therefore, we have $\mathfrak{c}\left(F_{\mathcal{B}}^{+}\right) u=\sum_{l} I_{l}\left\langle F_{\mathcal{B}}^{+}, \eta_{l}\right\rangle u$. Let $\left\{\zeta_{l}\right\}_{l=1}^{3}$ denote the basis elements of $\mathfrak{s p}(1)$. Then $(-u \bar{i}),(-u \bar{j})$ and $(-u \bar{k})$ are the fundamental vector fields at $u(\cdot)$ generated by $\zeta_{1}, \zeta_{2}$ and $\zeta_{3}$ respectively. We can write $u=-u \overline{i i}=-I_{1} u \bar{\zeta}_{1}$ and similarly for $j, \& k$.

$$
\begin{aligned}
\left\langle\mathfrak{c}\left(F_{\mathcal{B}}^{+}\right) u, u\right\rangle_{\mathbb{H}^{n+1}} & =\sum_{l}\left\langle I_{l}\left\langle F_{\mathcal{B}}^{+}, \eta_{l}\right\rangle u,-I_{l}\left(u \bar{\zeta}_{l}\right)\right\rangle_{\mathbb{H}^{n+1}} \\
& =\sum_{l}\left\langle\left\langle F_{\mathcal{B}}^{+}, \eta_{l}\right\rangle u, u \bar{\zeta}_{l}\right\rangle_{\mathbb{H}^{n+1}} \\
& =\left.2 \sum_{l} \mu_{S U(n+1)}\left(\left\langle F_{\mathcal{B}}^{+}, \eta_{l}\right\rangle \otimes \zeta_{l}\right)\right|_{u} \\
& =2\left\langle\mu_{S U(n+1)} \circ u, F_{\mathcal{B}}^{+}\right\rangle
\end{aligned}
$$

The claim follows by taking an inner product with a spinor $u$ on both sides of the eqn. (4.5.1) and from the above computations.

\subsection{Fueter operator and the generalized Dirac operator}

\subsubsection{Algebraic Preliminaries}

Let $\left(V, I_{1}, I_{2}, I_{3}\right)$ and $\left(W, J_{1}, J_{2}, J_{3}\right)$ be two quaternionic vector spaces. Let $f$ : $V \longrightarrow W$ be a linear map. Consider the $\mathbb{R}$-linear operator of $\mathbb{R}$-vector spaces

$$
\begin{gathered}
F: \operatorname{Hom}_{\mathbb{R}}(V, W) \longrightarrow \operatorname{Hom}_{\mathbb{R}}(V, W) \\
F(f)=\sum_{i=1}^{3} J_{k} \circ f \circ I_{k} .
\end{gathered}
$$


The operator $F$ is called Fueter operator. This was first introduced by R. Fueter [Fue35] in order to study the quaternionic analogue of Cauchy-Riemann equations.

Lemma 4.6.1. The Fueter operator satisfies the following properties:

1. $F^{2}+2 F-3=0$

2. The eigen-values of $F$ are $\{-3,1\}$.

It is a straight forward computation the verify (1) and claim (2) follows from (1). The Fueter operator decomposes the space $\operatorname{Hom}_{\mathbb{R}}(V, W)$ into a direct sum of two subspaces corresponding to the eigen-values $\{-3,1\}$. The maps corresponding to the eigen-value -3 are $\mathbb{H}$-linear.

Corollary 4.6.1. $(F-1) f$ is $\mathbb{H}$-linear for all $f \in H^{\circ o m} m_{\mathbb{R}}(V, W)$

Corresponding to the eigenvalues $\{-3,1\}$, we have the decompostion $H_{o m}(V, W)=$ $U_{-3} \oplus U_{+1}$. Observe that $(F+3)(F-1) f=0$. Therefore $(F-1) f \in U_{-3}$ and is $\mathbb{H}$-linear. We say that a map $f \in H_{\mathbb{R}}(V, W)$ is aholomorphic if $(F-1) f=0$.

\subsection{A brief survey on Aholomorphic maps}

\subsubsection{Stationary Harmonic maps}

Let $\left(M, g_{M}\right)$ and $\left(N, g_{N}\right)$ be any two compact Riemann manifolds. By Nash's isometric embedding theorem, we assume that $N$ is isometrically embedded in some $\mathbb{R}^{K}$. Let $N \hookrightarrow \mathcal{V} \subset \mathbb{R}^{K}$, be a neighbourhood of $N$ such that the nearest point projection $\Pi: \mathcal{V} \longrightarrow N$ is smooth and well-defined. Define the one parameter family of maps $u_{s}=\Pi(u+s \xi)$, where $\xi \in C^{\infty}\left(M, \mathbb{R}^{K}\right)$ and $s \in(-\varepsilon, \varepsilon)$ for some $\varepsilon>0$. Observe that for a small enough $s, u+s \xi \in \mathcal{V}$. Define $W^{1,2}(M, N)=\{f \in$ $W^{1,2}\left(M, \mathbb{R}^{K}\right) \mid f(x) \in N$ for a.e $\left.x \in M\right\}$. For $u \in W^{1,2}(M, N)$, define the energy functional

$$
E(u)=\frac{1}{2} \int_{M}\|d u\|^{2} d v o l_{M},
$$

where $d u: T M \longrightarrow \mathbb{R}^{K}$. Then $u$ is said to be weakly harmonic if for any $\xi \in$ $C^{\infty}\left(M, \mathbb{R}^{K}\right)$, we have

$$
\left.\frac{d}{d s} E\left(u_{s}\right)\right|_{s=0}=0
$$


in a weak sense. Or equivalently, is a weak solution of the associated EulerLagrange equation:

$$
\Delta u+A(u)(d u, d u)=0
$$

where, $\Delta$ is the usual Laplacian and $A(u)(d u, d u) \in\left(T_{u} N\right)^{\perp}$ is the second fundamental form on $N$. Observe that the system 4.7.2 is a non-linear system which is quadratic in first derivative. This non-linearity results in the loss of analytic properties of harmonic maps such as existence, regularity, etc. unless, we add some extra hypothesis. This is done by considering a second type of variation. Let $\phi_{t}: M \longrightarrow M$ be a $C^{1}$-family of diffeomorphisms such that $\phi_{0}=I d_{M}$. Let $u \in W^{1,2}(M, N)$ and $u_{s}=u \circ \phi_{s}$. Then $u$ is said to be stationary if for any family of diffeomorphisms $\left\{\phi_{t}\right\}$, satifying $\phi_{0}=I d_{M}$, it is a weak solution to

$$
\left.\frac{d}{d s} E\left(u_{s}\right)\right|_{s=0}=0
$$

In addition, if $u$ is weakly harmonic, then it is said to be stationary harmonic.

Theorem 4.7.1. [Sch84] Let $n=\operatorname{dim}_{\mathbb{R}} N$ and $u \in C^{2}\left(B_{\rho}(x), N\right)$, for some $x \in M$, be such that $E(u) \leq \Upsilon$. Then, there, exists some $\varepsilon>0$, depending only on $\Upsilon, n, N$, such that if

$$
\rho^{2-n} \int_{B_{\rho}(x)}\|d u\|^{2} d v o l_{M} \leq \varepsilon,
$$

then there exists a constant $C^{\prime}=C^{\prime}(\Upsilon, n, N)$ the following inequality holds:

$$
\sup _{B_{\rho / 2}(x)}\|d u\|^{2} \leq C^{\prime} \rho^{-n} \int_{B_{\rho}(x)}\|d u\|^{2} d v o l_{M} .
$$

Let $m=\operatorname{dim}_{\mathbb{R}} M$. Define the set $\mathscr{F}=\left\{u \in W^{1,2}(M, N) \mid E\left(u_{k}\right) \leq C\right\}$. Let $u_{k} \in \mathscr{F}$ be a sequence of maps converging weakly to a map $u \in W^{1,2}(M, N)$. Define the blow-up set of $\left\{u_{k}\right\}$ as in [Sch84]:

$$
\Sigma=\bigcap_{r>0}\left\{x \in M \mid \liminf _{k \rightarrow 0} r^{2-m} \int_{B_{r}(x)}\left\|d u_{k}\right\|^{2} d y \geq \varepsilon_{0}\right\}
$$

where the $\varepsilon_{0}$ is given by Theorem 4.7.1.

Corollary 4.7.1. [Sch84] The set $\Sigma$ is a closed singular set of locally finite Hausdorff measure $(m-2)$ in $M$ and any map $u$ in the weak $W^{1,2}$ - closure of $\mathscr{F}$ is smooth and harmonic on $M \backslash \Sigma$. 
Consider the Radon measure $\mu_{i}=\left\|d u_{i}\right\|^{2} d v o l_{M}$. Then without the loss of generality, we can assume that $\mu_{i} \rightarrow \mu$. Fatou's lemma ${ }^{2}$ implies $\mu=\|d u\|^{2} d v o l_{M}+$ $\nu$, where $\nu$ is some non-negative Radon measure. For a map $u \in W^{1,2}(M, N)$, define the regular and the singular sets by:

$$
\begin{gathered}
\operatorname{reg}(u)=\{x \in M \mid u \text { is regular in the neighbourhood of } x\} \\
\operatorname{sing}(u)=M \backslash \operatorname{reg}(u) .
\end{gathered}
$$

Theorem 4.7.2. [Lin99] The set $\Sigma$ is an $H^{m-2}$-rectifiable set ${ }^{3}$, where $H^{m-2}$ denotes the $m-2$-dimensional Hausdorff measure and:

1. $\Sigma=\operatorname{spt}(\nu) \cup \operatorname{sing}(u)$.

2. $\nu(x)=\Theta(x) H^{m-2}(\Sigma)$

where $\Theta$ is an $H^{m-2}$-measurable function.

Since $\Sigma$ is $H^{m-2}$-rectifiable, we can write it as a countable disjoint union $\Sigma=$ $\cup_{j=0}^{\infty} M_{j}$ where each $M_{j}$ for $j \geq 1$ is $H^{m-2}$ measurable, $H^{m-2}\left(M_{0}\right)=0$ and each $M_{j} \subset N_{j}$, where $N_{j}$ is an embedded $(n-2)$-dimensional $C^{1}$-submanifold of $\mathbb{R}^{m-2+k}$ for some $k \in \mathbb{N}$. Let $\mathscr{U}$ be an open subset of $\mathbb{R}^{m-2+k}$. For a locally Lipschitz function $f$ on $\mathscr{U}$, we can define the gradient operator $\nabla^{\Sigma} f, H^{m-2}$ a.e as

$$
\nabla^{\Sigma} f(x)=\nabla^{N_{j}} f(x)
$$

for $x \in M_{j}$ (cf. Section 12.1, [Sim83]) and hence the divergence operator div $v_{\Sigma} X=$ $\sum_{j=1}^{m-2}\left(\nabla_{e_{j}}^{\Sigma} X\right) \cdot e_{j}$, where $\left\{e_{j}\right\}_{j=1}^{m-2}$ is an orthornormal basis at $T_{x} \Sigma$ and $x \in \Sigma$. Here $T \Sigma$ is defined in the sense of Theorem 11.8 of [Sim83].

Theorem 4.7.3. [LT98] Let $u_{k} \in \mathscr{F}$ be a sequence of stationary harmonic maps such that $u_{k} \rightarrow u$ (weak convergence) in $W^{1,2}(M, N)$. Let $U \in M$ be an open set and let $X$ be a $C^{1}$-vector field on $M$ with compact support in $U$. Then

$$
\int_{\Sigma} \operatorname{div}_{\Sigma} X \nu+\int_{M}\left(\|d u\|^{2} \operatorname{div}(X)-2\left\langle d u\left(\nabla_{\alpha} X\right), d u\left(\frac{\partial}{\partial x^{\alpha}}\right)\right\rangle\right) d v o l_{M}=0
$$

\footnotetext{
${ }^{2}$ (Defect version of Fatou's lemma) Let $(X, B, \mu)$ be a measurable space and $\left\{f_{i}\right\}: X \longrightarrow[0,+\infty)$ be a sequence of measurable functions converging point-wise to an absolutely integrable function $f$. Then

$$
\int_{X} f_{n} d \mu-\int_{X} f d \mu-\left\|f_{n}-f\right\|_{L^{1}(\mu)} \rightarrow 0
$$

First informally proved in [BL86] by Brezis and Lieb.

${ }^{3}$ A set $M \in \mathbb{R}^{n+k}$ is said to be countably n-rectifiable, if $M \subset M_{0} \bigcup_{i=1}^{\infty} F_{i}\left(\mathbb{R}^{n}\right)$, where $F_{i}: \mathbb{R}^{n} \longrightarrow \mathbb{R}^{n+k}$ are Lipschitz continuous for $i=1,2,3, \ldots$.
} 
If a map $u \in W^{1,2}(M, N)$ is a stationary map, then ([Sch84]) it satisfies

$$
\sum_{\alpha} \int_{M}\left(\|d u\|^{2} \operatorname{div}(X)-2\left\langle d u\left(\nabla_{\alpha} X\right), d u\left(\frac{\partial}{\partial x^{\alpha}}\right)\right\rangle\right) d v o l_{M}=0 .
$$

But in general a weakly convergent sequence of stationary harmonic maps may not converge strongly and the energy may concentrate on the set $\Sigma$. We define the set $\Sigma$ to be stationary if

$$
\int_{\Sigma} \operatorname{div}_{\Sigma} X \nu=0
$$

for any vector field on $M$ with compact support. Thus the identity (4.7.5) and (4.7.6) implies that the singular set $\Sigma$ is stationary iff the limit map $u$ is a stationary map.

Theorem 4.7.4. [Bet93] Let $u \in W^{1,2}(M, N)$ be a stationary harmonic map. Then $H^{m-2}(\Sigma)=0$ and $u$ is smooth on $M \backslash \Sigma$.

\subsubsection{Aholomorphic maps between hyperKähler manifolds}

Let now $\left(M, g_{M}, I_{1}, I_{2}, I_{3}\right)$ and $\left(N, g_{N} J_{1}, J_{2}, J_{3}\right)$ be two compact hyperKähler manifolds.

Definition 4.7.1. A smooth map $u: M \longrightarrow N$ is said to be aholomorphic ${ }^{4}$ if $\sum_{i=1}^{3} J_{i} \circ d u \circ I_{i}=d u$.

Let $\left\{\omega_{I_{i}}\right\}_{i=1}^{3}$ and $\left\{\omega_{J_{i}}\right\}_{i=1}^{3}$ be the symplectic two-forms associated to the respective complex structures. Define the following energy functionals

$$
\begin{array}{r}
E(u)=\frac{1}{2} \int_{M}\|d u\|^{2} d v o l_{M}, \quad E_{\tau}(u)=\frac{1}{2} \sum_{i=1}^{3} \int_{M} \omega_{I_{i}} \wedge u^{*} \omega_{J_{i}} \\
I(u)=\frac{1}{2} \int_{M}\left\|d u-\sum_{i=1}^{3} J_{i} \circ d u \circ I_{i}\right\|^{2} d v o l_{M}=\frac{1}{2} \int_{M}\|(F-1) d u\|^{2} d v o l_{M}
\end{array}
$$

Then clearly, $u$ is an aholomorphic map iff $I(u)=0$.

\footnotetext{
${ }^{4}$ These maps are also known as quaternionic maps in some literature [CL00], [LT98], [Che99].
} 
Proposition 4.7.1. ([CLO0]) Let $M$ and $N$ be two hyperKähler manifolds as above and let $u: M \longrightarrow N$ be a smooth map. Then the energy functionals satisfy:

$$
\frac{1}{4} I(u)=E(u)+E_{\tau}(u)
$$

Furthermore, if $u$ is an aholomorphic map, then it minimizes the energy its homotopy class.

Remark 4.7.1. Being energy minimizers, aholomorphic maps are stationary harmonic maps. Therefore all of the results mentioned above carry over to aholomorphic maps. Additionally, it was proved by Schoen and Uhlenbeck [SU82] that for a sequence of energy-minimizing maps $u_{k}$, such that $u_{k} \rightarrow u$ in $W^{1,2}(M, N)$, the Hausdorff dimension of the singular set $\Sigma$ is at most $m-3$. It follows from 4.7.2 that $\nu=0$ and hence $\Sigma$ is stationary. Furthermore, Theorem from 4.7.3, it follows easily that the limit map $u$ is a stationary harmonic map.

Remark 4.7.2. If $M$ admits a permuting action of $S p(1)$, the $\mathfrak{s u}(2)$-valued symplectic two-form on $M$ is exact; i.e the cohomology class of each of the Kähler forms $\left\{\omega_{i}\right\}_{i=1}^{3}$ vanishes. Therefore $E_{\tau}(u)=0$. Furthermore, if $u$ is aholomorphic, $I(u)=0$ and therefore $E(u)=0$. Thus in this case, $u$ is a constant map.

\subsection{Aholomorphic maps and harmonic spinors}

Let $\left(X, g_{X}\right)$ be a four-dimensional Riemann manifold and endowed with a Spinstructure $Q \longrightarrow X$ and fix a connection $\mathrm{A} \in \mathscr{A}$ on $Q$. Let $M$ be a hyperKähler manifold with the permuting action of the group $\operatorname{Spin}_{\varepsilon}^{G}(4)$ and $u \in \mathscr{S}$ be a generalized spinor.

Clifford multiplication is the map $\mathfrak{m}:\left(\mathbb{R}^{4}\right)^{*} \otimes W^{0} \longrightarrow \epsilon_{-} \mathcal{C} l_{4} \epsilon_{+} \otimes_{\left(\mathcal{C l}_{4}^{0}\right)_{+}} W^{0}$. To define the generalized Dirac operator, we replace $W^{0}$ by $T M$. Note that having a permuting action of $\operatorname{Spin}_{\varepsilon}^{G}(4) \cong S p(1)+\times S p(1)_{-}$on $M$ implies that $S p(1)_{-}$acts trivial on $M$. Therefore (c.f Section 4.3):

$$
\mathcal{C} l_{4} \otimes_{\mathcal{C l} l_{4}^{0}} T M=\underbrace{T M}_{\mathrm{W}^{+}} \oplus \underbrace{\epsilon_{-} \mathcal{C} l_{4} \epsilon_{+} \otimes_{(\mathcal{C l})_{4}^{0}} T M}_{\mathrm{W}^{-}} .
$$

Let $u \in \mathscr{S}$ and $A \in \mathscr{A}$. Then composing the Clifford multiplication

$$
\mathfrak{m}:\left(\mathbb{R}^{4}\right)^{*} \otimes \mathrm{W}^{+} \longrightarrow \mathrm{W}^{-}
$$


with the covariant derivative of $u$,

$$
D_{\mathrm{A}} u \in C^{\infty}\left(Q,\left(\mathbb{R}^{4}\right)^{*} \otimes u^{*} \mathrm{~W}^{+}\right),
$$

we get the Dirac operator

$$
\mathfrak{D}_{\mathrm{A}}(u)=\sum_{i=0}^{3} e_{i} \cdot D_{\mathrm{A}} u\left(\tilde{e}_{i}\right) \text {. }
$$

Since $D_{\mathrm{A}} u\left(\tilde{e}_{i}\right) \in \mathbf{W}^{+}$, we have (Ref. Lemma 2.5.5)

$$
\mathscr{D}_{\mathrm{A}}(u)=\sum_{i=0}^{3} e_{i} \epsilon_{+} \cdot D_{\mathrm{A}} u\left(\tilde{e}_{i}\right)=\sum_{i=0}^{3} e_{0} \epsilon_{+} \lambda_{-\overline{e_{i}}} \cdot D_{\mathrm{A}} u\left(\tilde{e}_{i}\right)
$$

The element $\lambda_{e_{i}}$ acts on $T M$ as:

$$
\lambda_{e_{i}}=\left\{\begin{array}{l}
I_{i} \text { if } i=1,2,3 \\
I d_{T M} \text { if } i=0
\end{array}\right.
$$

and so

$$
e_{0} \epsilon_{+} \lambda_{-\overline{e_{i}}} \cdot D_{\mathrm{A}} u\left(\tilde{e_{i}}\right)= \begin{cases}e_{0} \epsilon_{+} \cdot I_{i} D_{\mathrm{A}} u\left(\tilde{e}_{i}\right) & \text { if } i=1,2,3 \\ -e_{0} \epsilon_{+} \cdot D_{\mathrm{A}} u\left(\tilde{e_{i}}\right) \text { if } i=0 .\end{cases}
$$

Rewriting,

$$
\begin{aligned}
\mathfrak{P}_{\mathrm{A}}(u) & =\sum_{i=0}^{3} e_{i} \cdot D_{\mathrm{A}} u\left(\tilde{e}_{i}\right) \\
& =e_{0} \epsilon_{+} \cdot\left(\sum_{i=1}^{3} I_{i} D_{\mathrm{A}} u\left(\tilde{e_{i}}\right)-D_{\mathrm{A}}\left(\tilde{e_{0}}\right)\right) \\
& =e_{0} \epsilon_{+} \cdot\left(\sum_{i=1}^{3} I_{i} D_{\mathrm{A}} u\left(I_{i} \tilde{e_{0}}\right)-D_{\mathrm{A}}\left(\tilde{e_{0}}\right)\right) \\
& =e_{0} \epsilon_{+} \cdot\left(F\left(D_{\mathrm{A}} u\right)\left(\tilde{e_{0}}\right)-D_{\mathrm{A}} u\left(\tilde{e_{0}}\right)\right) \\
& =e_{0} \epsilon_{+} \cdot\left((F-1) D_{\mathrm{A}} u\left(\tilde{e_{0}}\right)\right) .
\end{aligned}
$$

The above computations were presented in [Pid13].

Definition 4.8.1. We say that a generalized spinor is aholomorphic if

$$
(F-1) D_{\mathrm{A}} u\left(\tilde{e_{0}}\right)=0 .
$$


Thus, from the computations above, we see that if $u$ is harmonic, then $u$ is aholomorphic.

Remark 4.8.1. Suppose that the base manifold $\left(X, J_{1}, J_{2}, J_{3}\right)$ is a hyperKähler manifold and the group $G$ is trivial. Since $S p(1)_{-}$acts trivially on $M$, the generalized spinors are maps from $Q / S p(1)_{-} \cong P_{S O(3)_{+}} \longrightarrow M$, where $\pi: P_{S O(3)_{+}} \longrightarrow X$ is the principal bundle associated to $\Lambda_{+}^{2} X$. For any $x \in X$, hyperKähler structure on $X$ defines a distinguished frame $\left.\zeta\right|_{x} \in \pi^{-1}(x)$, given by the symplectic twoforms $\omega_{J_{i}}^{X}$. Define the trivialization by $P_{S O(3)_{+}} \ni p \mapsto(\pi(p), g) \in X \times S O(3)_{+}$, where $p=\left.\zeta\right|_{x} \cdot g$. Let $u: P_{S O(3)_{+}} \longrightarrow M$ be a generalized spinor. Define the map $\tilde{u}: X \longrightarrow M$ by $\tilde{u}(x)=u\left(\left.\zeta\right|_{x}\right)$. Then $u$ is just an $S O(3)$-equivariant extension of $\tilde{u}$. Consider the trivial connection $\mathrm{A}_{\text {triv }}$ on $P_{S O(3)_{+}}$. The covariant derivative associated to the trivial connection is $D_{\mathrm{A}_{\text {triv }}} u=T u \circ \mathrm{pr}_{\mathcal{H}_{\mathrm{A}_{\text {triv }}}}=T \tilde{u}$. Therefore harmonic spinors are maps satisfying $(F-1) T \tilde{u}=0$. In other words, harmonic spinors are equivariant lifts of aholomorphic maps $u: X \longrightarrow M$.

Theorem 4.8.1. [Pid04] (Weitzenböck formula) Let $X$ be a four-dimensional, compact, Riemann manifold. Let $\theta_{X}$ denote the canonical one-form on $P_{S O(4)}$ and $\omega$ denote the $\mathfrak{s u}(2)$-valued symplectic, two-form on the target hyperKähler manifold M. Then

$\int_{X}\left\|\mathscr{D}_{\mathrm{A}} u\right\|^{2} d v o l_{X}=\int_{X} u^{*} g_{M} \otimes g_{X}\left(D_{\mathrm{A}} u, D_{A} u\right) d v o l_{X}+\int_{X} \pi_{!}\left(\left\langle\left(u^{*} \omega\right)_{h o r} \wedge\left(\theta_{X} \wedge \theta_{X}^{T}\right)_{+}\right\rangle_{\mathfrak{s u}(2)}\right)$

where $\theta_{X}^{T}$ denotes the transpose of $\theta_{X},\left(\theta_{X} \wedge \theta_{X}^{T}\right)_{+}$denotes the self-dual part of the two-form $\left(\theta_{X} \wedge \theta_{X}^{T}\right)$ and $\pi_{!}$is an isomorphism between $\Omega^{*}(Q)_{h o r}^{\operatorname{Spin}_{\varepsilon}^{G}(4)}$ and $\Omega^{*}(X)$.

Observe that

$$
\begin{aligned}
g_{M}\left((F-1) D_{\mathrm{A}} u,(F-1) D_{\mathrm{A}} u\right) d v o l_{X} & =\sum_{i=0}^{3} g_{M}\left((F-1) D_{\mathrm{A}} u\left(\tilde{e}_{i}\right),(F-1) D_{\mathrm{A}} u\left(\tilde{e}_{i}\right)\right) \\
& =4 g_{M}\left(\mathscr{D}_{\mathrm{A}}(u), \mathscr{D}_{\mathrm{A}}(u)\right) .
\end{aligned}
$$

Define the energy functionals

$$
\begin{gathered}
E(u, \mathrm{~A})=\frac{1}{2} \int_{X} u^{*} g_{M} \otimes g_{X}\left(D_{\mathrm{A}} u, D_{\mathrm{A}} u\right) d v o l_{X}, \\
E_{\tau}(u, \mathrm{~A})=\frac{1}{2} \int_{X} \pi_{!}\left(\left\langle\left(u^{*} \omega\right)_{h o r} \wedge\left(\theta_{X} \wedge \theta_{X}^{T}\right)_{+}\right\rangle_{\mathfrak{s u}(2)}\right), \quad I(u, \mathrm{~A})=\frac{1}{2} \int_{X}\left\|\mathscr{P}_{\mathrm{A}} u\right\|^{2} d v o l_{X}
\end{gathered}
$$


From the identity 4.8.1 we see that the spinors satisfy an analogue of the energy identity 4.7 .8 for aholomorphic maps.

Remark 4.8.2. Consider again the case where $\left(X, J_{1}, J_{2}, J_{3}\right)$ is a hyperKähler manifold (cf. Remark 4.8.1). Let $\mathrm{A}^{\prime}$ is some non-trivial connection on $P_{\mathrm{SO}(3)_{+}}$. Since $\left(\theta_{X} \wedge \theta_{X}\right)_{+} \in \Omega^{1}\left(P_{S O(3)_{+}}, \Lambda_{+}^{2} \mathbb{R}^{4}\right)$ and $X$ is hyperKähler, $\left(\theta_{X} \wedge \theta_{X}\right)_{+}=\pi^{*} \omega^{X}$, where $\omega^{X}$ is the $\mathfrak{s u}(2)$-valued symplectic two-form on $X$. Therefore,

$$
\begin{aligned}
E_{\tau}\left(u, \mathrm{~A}^{\prime}\right) & =\frac{1}{2} \int_{X} \pi_{!}\left(\left\langle\left(u^{*} \omega\right)_{h o r} \wedge\left(\theta_{X} \wedge \theta_{X}^{T}\right)_{+}\right\rangle_{\mathfrak{s u}(2)}\right) \\
& =\frac{1}{2} \int_{X} \pi_{!}\left(\left\langle\left(u^{*} \omega\right)_{h o r} \wedge \pi^{*} \omega^{X}\right\rangle_{\mathfrak{s u}(2)}\right)
\end{aligned}
$$

Since $M$ admits a permuting $S p(1)$ action, the two-form $u^{*} \omega$ is exact and $u^{*} \omega=$ $d\left(u^{*} \gamma_{1}\right)$. Given any two vector fields $Z, Y \in \Gamma\left(P_{S O(3)_{+}}, T P_{S O(3)_{+}}\right)$, we have:

$$
\begin{aligned}
d\left(u^{*} \gamma_{1}\right)_{h o r}(Z, Y)-\left(d\left(u^{*} \gamma_{1}\right)\right)_{h o r}(Z, Y) & =(Z-\tilde{Z})\left(u^{*} \gamma_{1}\right)(\tilde{Y})-(Y-\tilde{Y})\left(u^{*} \gamma_{1}\right)(\tilde{Z}) \\
& -\left(u^{*} \gamma_{1}\right)([Z, Y]-\widetilde{[Z, Y]})
\end{aligned}
$$

where $\tilde{Z}, \tilde{Y}, \widetilde{[Z, Y]}$ denote the horizontal parts of the respective vector fields with respect to the connection $A^{\prime}$. We see therefore that the $d\left(u^{*} \gamma_{1}\right)_{\text {hor }} \neq\left(d\left(u^{*} \gamma_{1}\right)\right)_{\text {hor }}$ and the energy $E_{\tau}\left(u, \mathrm{~A}^{\prime}\right)$ may not be invariant under the homotopy class of $u$.

Let now $\mathrm{A}^{\prime}=\mathrm{A}_{\text {triv }}$ and consider the following commutative diagram, where $u, \tilde{u}$ are as defined in Remark 4.8.1. Let $\pi_{\text {triv }}$ denote the trivialization of $P_{S O(3)_{+}}$as described in the same remark.

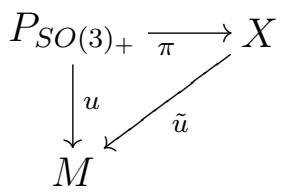

Since the connection is trivial, $\Omega^{*}\left(P_{S O(3)+}\right)_{h o r}^{\operatorname{Spin}_{\varepsilon}^{G}(4)}=\pi_{\text {triv }}^{*}\left(\Omega^{*}(X)\right)=\pi^{*}\left(\Omega^{*}(X)\right)$. Therefore for any two vector fields $Z, Y \in \Gamma\left(P_{S O(3)_{+}}, T P_{S O(3)_{+}}\right)$,

$$
\begin{aligned}
\left(u^{*} \omega\right)_{h o r}(Z, Y) & =\left(u^{*} \omega\right)(\tilde{Z}, \tilde{Y})=\left((\tilde{u} \circ \pi)^{*} \omega\right)(\tilde{Z}, \tilde{Y}) \\
& =\left(\tilde{u}^{*} \omega\right)(T \pi(\tilde{Z}), T \pi(\tilde{Y}))=\pi^{*}\left(\left(\tilde{u}^{*} \omega\right)\right)(Z, Y) .
\end{aligned}
$$

Note that the last step uses the triviality of the connection. Therefore

$$
\pi_{!}\left(\left\langle\left(u^{*} \omega\right)_{h o r} \wedge \pi^{*} \omega^{X}\right\rangle_{\mathfrak{s u}(2)}\right)=\pi_{!}\left(\left\langle\pi^{*}\left(\tilde{u}^{*} \omega\right)_{h o r} \wedge \pi^{*} \omega^{X}\right\rangle_{\mathfrak{s u}(2)}\right)=\left\langle\tilde{u}^{*} \omega \wedge \omega^{X}\right\rangle_{\mathfrak{s u}(2)}
$$


and therefore, for a trivial connection $\mathrm{A}_{\text {triv }}$,

$$
E_{\tau}\left(u, \mathrm{~A}_{\text {triv }}\right)=\frac{1}{2} \int_{X}\left(\left\langle\left(\tilde{u}^{*} \omega\right) \wedge \omega^{X}\right\rangle_{\mathfrak{s u}(2)}\right)=\frac{1}{2} \sum_{i=1}^{3} \int_{X}\left(\tilde{u}^{*} \omega_{I_{i}}\right) \wedge \omega_{J_{i}}^{X} .
$$

Let $\psi: T T M \longrightarrow T M$ be a torsion-free connector. Define the first order differential operator:

$$
\begin{gathered}
\nabla^{\mathrm{A}, \psi}: C^{\infty}(P, T M)^{\operatorname{Spin}_{\varepsilon}^{G}(4)} \longrightarrow \operatorname{Hom}(T P, T M)_{h o r}^{\operatorname{Spin}_{\varepsilon}^{G}(4)} \\
v \longrightarrow \psi \circ T v \circ \operatorname{pr}_{\mathcal{H}_{\mathrm{A}}}
\end{gathered}
$$

This coincides with the linearization of the covariant derivative [Sch10].

In order to derive a condition analogous to 4.7.6, for the spinor being stationary, we analyze the behavior of the energy functionals under the deformations on the source manifold. Let $\phi_{t}: X \longrightarrow X$, for $t \in[0,1]$, be a family of diffeomorphisms of $X$. Let $\hat{\phi}_{t}$ denote the horizontal lift of $\phi_{t}$ to $Q$ w.r.t a fixed connection A on $Q$. Note that, since the energy functionals are gauge-invariant, we may assume that $\hat{\phi}_{0}=$ Id. For if not, then we consider the family $\hat{\phi}_{t} \circ \hat{\phi}_{0}^{-1}$. Define $u_{t}=u \circ \hat{\phi}_{t}$ and let $Y=\left.\frac{d}{d t} \hat{\phi}_{t}^{*}\right|_{t=0}$. Then,

$$
E_{\tau}\left(u_{t}\right)=\frac{1}{2} \int_{X} \pi_{!}\left(\left\langle\left(u_{t}^{*} \omega\right)_{h o r} \wedge\left(\theta_{X} \wedge \theta_{X}^{T}\right)_{+}\right\rangle_{\mathfrak{s u}(2)}\right)=\frac{1}{2} \int_{X} \pi_{!}\left(\left\langle\left(\hat{\phi}_{t}^{*} u^{*} \omega\right)_{h o r} \wedge\left(\theta_{X} \wedge \theta_{X}^{T}\right)_{+}\right\rangle_{\mathfrak{s u}(2)}\right)
$$

Therefore,

$$
\begin{aligned}
\left.\frac{d}{d t} E_{\tau}\left(u_{t}\right)\right|_{t=0} & =\left.\frac{1}{2} \frac{d}{d t} \int_{X} \pi_{!}\left(\left\langle\left(\hat{\phi}_{t}^{*} u^{*} \omega\right)_{h o r} \wedge\left(\theta_{X} \wedge \theta_{X}^{T}\right)_{+}\right\rangle_{\mathfrak{s u}(2)}\right)\right|_{t=0} \\
& =\frac{1}{2} \int_{X} \pi_{!}\left(\left\langle\left.\frac{d}{d t}\left(\hat{\phi}_{t}^{*} u^{*} \omega\right)_{h o r}\right|_{t=0} \wedge\left(\theta_{X} \wedge \theta_{X}^{T}\right)_{+}\right\rangle_{\mathfrak{s u}(2)}\right) \\
& =\frac{1}{2} \int_{X} \pi_{!}\left(\left\langle\left(\mathcal{L}_{\left.\frac{d}{d t} \hat{\phi}_{t}^{*}\right|_{t=0}} u^{*} \omega\right)_{h o r} \wedge\left(\theta_{X} \wedge \theta_{X}^{T}\right)_{+}\right\rangle_{\mathfrak{s u}(2)}\right) \\
& =\frac{1}{2} \int_{X} \pi_{!}\left(\left\langle\left(d \iota_{Y} u^{*} \omega\right)_{h o r} \wedge\left(\theta_{X} \wedge \theta_{X}^{T}\right)_{+}\right\rangle_{\mathfrak{s u}(2)}\right)
\end{aligned}
$$

Given a one form $\alpha \in \Omega^{1}(Q)$, we can write it as sum of horizontal and vertical components: $\alpha=\alpha_{\text {hor }}+\alpha_{\text {vert }}$, where $\alpha_{\text {hor }}=\alpha \circ \operatorname{pr}_{\mathcal{H}_{\mathrm{A}}}$ and $\alpha_{\text {vert }}=\left\langle\mathrm{A}, \iota_{\mathfrak{s p i n}_{\epsilon}^{G}(4)} \alpha\right\rangle:=\iota_{K_{\mathrm{A}}} \alpha$. Therefore,

$$
\begin{aligned}
& \left.\frac{d}{d t} E_{\tau}\left(u_{t}\right)\right|_{t=0} \\
& =\frac{1}{2} \int_{X} \pi_{!}\left(\left\langle\left(d\left(\iota_{Y} u^{*} \omega\right)_{h o r}+\left(\iota_{Y} u^{*} \omega\right)_{\text {vert }}\right)_{h o r} \wedge\left(\theta_{X} \wedge \theta_{X}^{T}\right)_{+}\right\rangle_{\mathfrak{s u}(2)}\right)
\end{aligned}
$$




$$
\begin{aligned}
& =\frac{1}{2} \int_{X} \pi_{!}\left(\left\langle\left(d\left(\iota_{Y} u^{*} \omega\right)_{h o r}\right)_{h o r} \wedge\left(\theta_{X} \wedge \theta_{X}^{T}\right)_{+}\right\rangle_{\mathfrak{s u}(2)}\right) \\
& +\frac{1}{2} \int_{X} \pi_{!}\left(\left\langle\left(d\left(\iota_{Y} u^{*} \omega\right)_{v e r t}\right)_{h o r} \wedge\left(\theta_{X} \wedge \theta_{X}^{T}\right)_{+}\right\rangle_{\mathfrak{s u}(2)}\right) \\
& =\frac{1}{2} \int_{X} \pi_{!}\left(\left\langle D_{\mathrm{A}}\left(\iota_{Y} u^{*} \omega\right) \wedge\left(\theta_{X} \wedge \theta_{X}^{T}\right)_{+}\right\rangle_{\mathfrak{s u}(2)}\right)+\frac{1}{2} \int_{X} \pi_{!}\left(\left\langle\left(d\left(\iota_{Y} u^{*} \omega\right)_{\text {vert }}\right)_{\text {hor }} \wedge\left(\theta_{X} \wedge \theta_{X}^{T}\right)_{+}\right\rangle_{\mathfrak{s u}(2)}\right)
\end{aligned}
$$

The one form

$$
\begin{aligned}
\left(\iota_{Y} u^{*} \omega\right)_{\text {vert }} & =\left\langle\mathrm{A}, \iota_{\mathfrak{s p i n}_{\epsilon}^{G}(4)} \iota_{Y} u^{*} \omega\right\rangle=-\left\langle\mathrm{A}, \iota_{Y} \iota_{\mathfrak{s p i n}_{\epsilon}^{G}(4)} u^{*} \omega\right\rangle=-\left\langle\mathrm{A}, \iota_{Y} u^{*}\left(\iota_{\mathfrak{s p i n}_{\epsilon}^{G}(4)} \omega\right)\right\rangle \\
& =-\left\langle\varphi_{+}, \iota_{Y} u^{*}\left(\iota_{\mathfrak{s p}(1)} \omega\right)\right\rangle_{\mathfrak{s u}(2)}-\left\langle\mathrm{B}, \iota_{Y} u^{*}\left(\iota_{\mathfrak{g}} \omega\right)\right\rangle_{\mathfrak{g}} \\
& \left.\left.=-\left\langle\varphi_{+}, \iota_{Y} u^{*} \gamma\right)\right\rangle_{\mathfrak{s u}(2)}-\left\langle\mathrm{B}, \iota_{Y} u^{*} d \mu_{G}\right)\right\rangle_{\mathfrak{g}}
\end{aligned}
$$

where B denotes the connection on $P_{G}, \varphi_{+}$is the $\mathfrak{s u}(2)_{+}$component of the LeviCivita connection on $X$ and $\mu_{G}$ is the momentum map for the hyperKähler action of $G$ on $M$. Therefore

$$
\begin{aligned}
d\left(\iota_{Y} u^{*} \omega\right)_{\text {vert }} & =-d\left\langle\varphi_{+}, \iota_{Y} u^{*} \gamma\right\rangle_{\mathfrak{s u}(2)}-d\left\langle\mathrm{~B}, \iota_{Y} u^{*} d \mu_{G}\right\rangle_{\mathfrak{g}} \\
& =-\left\langle d \varphi_{+}, \iota_{Y} u^{*} \gamma\right\rangle_{\mathfrak{s u}(2)}-\left\langle\varphi_{+}, d \iota_{Y} u^{*} \gamma\right\rangle_{\mathfrak{s u}(2)}-\left\langle d \mathrm{~B}, \iota_{Y} u^{*} d \mu_{G}\right\rangle_{\mathfrak{g}}-\left\langle\mathrm{B}, d \iota_{Y} u^{*} d \mu_{G}\right\rangle_{\mathfrak{g}}
\end{aligned}
$$

We can further simplify the above expression:

$$
\left\langle d \varphi_{+}, \iota_{Y} u^{*} \gamma\right\rangle_{\mathfrak{s u}(2)}=\left\langle F_{\varphi_{+}}, \iota_{Y} u^{*} \gamma\right\rangle_{\mathfrak{s u}(2)}-\left\langle\varphi_{+} \wedge \varphi_{+}, \iota_{Y} u^{*} \gamma\right\rangle_{\mathfrak{s u}(2)}
$$

Similarly,

$$
\left\langle d \mathrm{~B}, \iota_{Y} u^{*} d \mu_{G}\right\rangle_{\mathfrak{g}}=\left\langle F_{\mathrm{B}}, \iota_{Y} u^{*} \gamma\right\rangle_{\mathfrak{g}}-\left\langle\mathrm{B} \wedge \mathrm{B}, \iota_{Y} u^{*} \gamma\right\rangle_{\mathfrak{g}}
$$

Therefore combining the above expressions, we get

$$
\left(d\left(\iota_{Y} u^{*} \omega\right)_{v e r t}\right)_{h o r}=-\left\langle F_{\varphi_{+}}, \iota_{Y} u^{*} \gamma\right\rangle_{\mathfrak{s u}(2)}-\left\langle F_{\mathrm{B}}, \iota_{Y} u^{*} \gamma\right\rangle_{\mathfrak{g}}
$$

since

1. $\left.\left(\left\langle\varphi_{+}, d \iota_{Y} u^{*} \gamma\right\rangle\right)_{h o r}=\left\langle\mathrm{B}, d \iota_{Y} u^{*} d \mu_{G}\right\rangle\right)_{h o r}=0$,

2. $\left\langle F_{\phi_{+}}, \iota_{Y} u^{*} \gamma\right\rangle_{h o r}=\left\langle F_{\phi_{+}}, \iota_{Y} u^{*} \gamma\right\rangle$

3. $\left\langle F_{\mathrm{B}}, \iota_{Y} u^{*} d \mu_{G}\right\rangle_{h o r}=\left\langle F_{\mathrm{B}}, \iota_{Y} u^{*} d \mu_{G}\right\rangle=\left\langle F_{\mathrm{B}}, \iota_{Y} d\left(\mu_{G} \circ u\right)\right\rangle$. 
Note that since $S U(2)_{-}$acts trivially on $M$, only the $\mathfrak{s u}(2)_{+}$component of the Levi-Civita connection survives. Substituting this, we get:

$\left.\frac{d}{d t} E_{\tau}\left(u_{t}\right)\right|_{t=0}$

$=\frac{1}{2} \int_{X} \pi_{!}\left(\left\langle D_{\mathrm{A}}\left(\iota_{Y} u^{*} \omega\right) \wedge\left(\theta_{X} \wedge \theta_{X}^{T}\right)_{+}\right\rangle_{\mathfrak{s u}(2)}\right)-\frac{1}{2} \int_{X} \pi_{!}\left(\left\langle\left(d\left\langle\varphi_{+}, \iota_{Y} u^{*} \gamma\right\rangle\right)_{h o r} \wedge\left(\theta_{X} \wedge \theta_{X}^{T}\right)_{+}\right\rangle_{\mathfrak{s u}(2)}\right)$

$-\frac{1}{2} \int_{X} \pi_{!}\left(\left\langle\left(d\left\langle\mathrm{~B}, \iota_{Y} u^{*} d \mu_{G}\right\rangle\right)_{h o r} \wedge\left(\theta_{X} \wedge \theta_{X}^{T}\right)_{+}\right\rangle_{\mathfrak{s u}(2)}\right)$

$=\frac{1}{2} \int_{X} \pi_{!}\left(\left\langle D_{\mathrm{A}}\left(\iota_{Y} u^{*} \omega\right) \wedge\left(\theta_{X} \wedge \theta_{X}^{T}\right)_{+}\right\rangle_{\mathfrak{s u}(2)}\right)-\frac{1}{2} \int_{X} \pi_{!}\left(\left\langle\left(\left\langle F_{\varphi_{+}}, \iota_{Y} u^{*} \gamma\right\rangle\right) \wedge\left(\theta_{X} \wedge \theta_{X}^{T}\right)_{+}\right\rangle_{\mathfrak{s u}(2)}\right)$

$-\frac{1}{2} \int_{X} \pi_{!}\left(\left\langle\left(\left\langle F_{\mathrm{B}}, \iota_{Y} u^{*} d \mu_{G}\right\rangle\right)_{h o r} \wedge\left(\theta_{X} \wedge \theta_{X}^{T}\right)_{+}\right\rangle_{\mathfrak{s u}(2)}\right)$

The first term in the above expression can be re-written as

$$
\begin{aligned}
\frac{1}{2} \int_{X} \pi_{!}\left(\left\langle D_{\mathrm{A}}\left(\iota_{Y} u^{*} \omega\right) \wedge\left(\theta_{X} \wedge \theta_{X}^{T}\right)_{+}\right\rangle_{\mathfrak{s u}(2)}\right) & =\frac{1}{2} \int_{X} d\left(\pi_{!}\left(\left\langle\left(\iota_{Y} u^{*} \omega \wedge\left(\theta_{X} \wedge \theta_{X}^{T}\right)_{+}\right\rangle_{\mathfrak{s u}(2)}\right)\right)\right. \\
& -\frac{1}{2} \int_{X} \pi_{!}\left(\left\langle\left(\iota_{Y} u^{*} \omega \wedge D_{\mathrm{A}}\left(\theta_{X} \wedge \theta_{X}^{T}\right)_{+}\right)\right\rangle_{\mathfrak{s u}(2)}\right)
\end{aligned}
$$

The first term vanishes since the base manifold $X$ is closed and compact. The second term vanishes sinces the Levi-Civita connection is torsionless. Therefore,

$$
\begin{aligned}
\left.\frac{d}{d t} E_{\tau}\left(u_{t}\right)\right|_{t=0} & =-\frac{1}{2} \int_{X} \pi_{!}\left(\left\langle\left(\left\langle F_{\varphi_{+}}, \iota_{Y} u^{*} \gamma\right\rangle\right) \wedge\left(\theta_{X} \wedge \theta_{X}^{T}\right)_{+}\right\rangle_{\mathfrak{s u}(2)}\right) \\
& -\frac{1}{2} \int_{X} \pi_{!}\left(\left\langle\left(\left\langle F_{\mathrm{B}}, \iota_{Y} d\left(\mu_{G} \circ u\right)\right\rangle\right)_{h o r} \wedge\left(\theta_{X} \wedge \theta_{X}^{T}\right)_{+}\right\rangle_{\mathfrak{s u}(2)}\right)
\end{aligned}
$$

The first summand can be further simplified as follows:

$$
\begin{aligned}
& \frac{1}{2} \int_{X} \pi_{!}\left(\left\langle\left(\left\langle F_{\varphi_{+}}, \iota_{Y} u^{*} \gamma\right\rangle\right) \wedge\left(\theta_{X} \wedge \theta_{X}^{T}\right)_{+}\right\rangle_{\mathfrak{s u}(2)}\right) \\
& =\frac{1}{2} \int_{X} \pi_{!}\left(\left\langle\left(\frac{(-1)}{3} \iota_{Y} u^{*} \gamma_{0} F_{\varphi_{+}}-\left[\iota_{Y} u^{*} \gamma_{1}, F_{\varphi_{+}}\right]\right) \wedge\left(\theta_{X} \wedge \theta_{X}^{T}\right)_{+}\right\rangle_{\mathfrak{s u}(2)}\right) \\
& =\frac{1}{2} \int_{X} \pi_{!} \operatorname{tr}\left(\left(\left\langle\left(\frac{(-1)}{3} \iota_{Y} u^{*} \gamma_{0} F_{\varphi_{+}}-\left[\iota_{Y} u^{*} \gamma_{1}, F_{\varphi_{+}}\right]\right) \wedge\left(\theta_{X} \wedge \theta_{X}^{T}\right)_{+}\right)\right.\right. \\
& =\frac{1}{2} \int_{X} \pi_{!}\left[\left(\frac{(-1)}{3} \iota_{Y} u^{*} \gamma_{0}\right) \operatorname{tr}\left(\left(\mathcal{W}_{\varphi}^{+}+\frac{s_{X}}{12}\left(\theta_{X} \wedge \theta_{X}^{T}\right)_{+}\right) \wedge\left(\theta_{X} \wedge \theta_{X}^{T}\right)_{+}\right)\right] \\
& -\frac{1}{2} \int_{X} \pi_{!}\left[\operatorname{tr}\left(\left[\left(\iota_{Y} u^{*} \gamma_{1}\right), \mathcal{W}_{\varphi}^{+}+\frac{s_{X}}{12}\left(\theta_{X} \wedge \theta_{X}^{T}\right)_{+}\right]\right) \wedge\left(\theta_{X} \wedge \theta_{X}^{T}\right)_{+}\right]
\end{aligned}
$$


$=\frac{(-1)}{24} \int_{X} \pi_{!}\left(\left(\iota_{Y} u^{*} \gamma_{0}\right) s_{X}\right) d v o l_{X}-\frac{1}{2} \int_{X} \pi_{!}\left(\operatorname{tr}\left(\left[\left(\iota_{Y} u^{*} \gamma_{1}\right), \mathcal{W}_{\varphi}^{+}\right] \wedge\left(\theta_{X} \wedge \theta_{X}^{T}\right)_{+}\right)\right) d v o l_{X}$

Consider now the energy functional $E\left(u_{t}, \mathrm{~A}_{t}\right)$, where $\mathrm{A}_{t}=\hat{\phi}_{t}^{*}(\mathrm{~A})$. Since the the space of connections is an affine space, we can equivalently write $\hat{\phi}_{t}^{*}(\mathrm{~A})=\mathrm{A}+\alpha_{t}$. Therefore

$\left.\frac{d}{d t} E\left(u_{t}, \mathrm{~A}_{t}\right)\right|_{t=0}$

$=\left.\frac{d}{d t}\left(\frac{1}{2} \int_{X} g_{M} \otimes g_{X}\left(D_{\mathrm{A}_{t}} u_{t}, D_{\mathrm{A}_{t}} u_{t}\right) d v o l_{X}\right)\right|_{t=0}=\int_{X} g_{M} \otimes g_{X}\left(D_{\mathrm{A}} u,\left.\frac{d}{d t} D_{\mathrm{A}_{t}} u_{t}\right|_{t=0}\right) d v o l_{X}$

$=\int_{X} g_{M} \otimes g_{X}\left(D_{\mathrm{A}} u,\left.\frac{d}{d t} D_{\mathrm{A}} u_{t}\right|_{t=0}\right) d v o l_{X}+\int_{X} g_{M} \otimes g_{X}\left(D_{\mathrm{A}} u,\left.\left.\frac{d}{d t} K_{\alpha_{t}}\right|_{u_{t}}\right|_{t=0}\right) d v o l_{X}$

$=\int_{X} g_{M}\left(\nabla^{A, \psi, *}\left(D_{\mathrm{A}} u\right), d u(Y)\right) d v o l_{X}+\int_{X} g_{M} \otimes g_{X}\left(D_{\mathrm{A}} u,\left.\left.\frac{d}{d t} K_{\alpha_{t}}^{M}\right|_{u_{t}}\right|_{t=0}\right) d v o l_{X}$

For every $m \in M$, we have the map $\left.K^{M}\right|_{m}: \mathfrak{s p i n}(4)_{\varepsilon}^{G} \longrightarrow T_{m} M$ whose adjoint is $\left.\left(K^{M}\right)^{*}\right|_{m}: T_{m} M \longrightarrow \mathfrak{s p i n}(4)_{\varepsilon}^{G}$. Therefore we have:

$\left.\frac{d}{d t} E\left(u_{t}, \mathrm{~A}_{t}\right)\right|_{t=0}$

$=\int_{X} g_{M}\left(\nabla^{A, \psi, *}\left(D_{\mathrm{A}} u\right), d u(Y)\right) d v o l_{X}+\int_{X} g_{M} \otimes g_{X}\left(D_{\mathrm{A}} u,\left.\left.\frac{d}{d t} K_{\alpha_{t}}^{M}\right|_{u_{t}}\right|_{t=0}\right) d v o l_{X}$

$=\int_{X} g_{M}\left(\nabla^{A, \psi, *}\left(D_{\mathrm{A}} u\right), d u(Y)\right) d v o l_{X}+\int_{X} g_{M} \otimes g_{X}\left(D_{\mathrm{A}} u,\left.\left.\frac{d}{d t} K_{\alpha_{t}}^{M}\right|_{u}\right|_{t=0}\right) d v o l_{X}$

$=\int_{X} g_{M}\left(\nabla^{A, \psi, *}\left(D_{\mathrm{A}} u\right), d u(Y)\right) d v o l_{X}+\int_{X}\left\langle\left.\left(K^{M}\right)^{*}\right|_{m}\left(D_{\mathrm{A}} u\right),\left.\frac{d}{d t} K_{\alpha_{t}}\right|_{t=0}\right\rangle_{T^{*} X \otimes \mathfrak{s p i n}(4)_{\varepsilon}^{G}} d v o l_{X}$

$=\int_{X} g_{M}\left(\nabla^{A, \psi, *}\left(D_{\mathrm{A}} u\right), d u(Y)\right) d v o l_{X}+\int_{X}\left\langle\left.\left(K^{M}\right)^{*}\right|_{m}\left(D_{\mathrm{A}} u\right), \mathcal{L}_{Y} \mathrm{~A}\right\rangle_{T^{*} X \otimes \mathfrak{s p i n}(4)_{\varepsilon}^{G}} d v o l_{X}$

$=\int_{X} g_{M}\left(\nabla^{A, \psi, *}\left(D_{\mathrm{A}} u\right), d u(Y)\right) d v o l_{X}+\int_{X}\left\langle\left.\left(K^{M}\right)^{*}\right|_{m}\left(D_{\mathrm{A}} u\right), \iota_{Y} F_{\mathrm{A}}\right\rangle_{T^{*} X \otimes \mathfrak{s p i n}(4)_{\varepsilon}^{G}} d v o l_{X}$

$=\int_{X} g_{M}\left(\nabla^{A, \psi, *}\left(D_{\mathrm{A}} u\right), d u(Y)\right) d v o l_{X}+\int_{X} g_{M} \otimes g_{X}\left(D_{\mathrm{A}} u, K_{\iota_{Y} F_{\mathrm{A}}}^{M}\right) d v o l_{X}$

Combining the computations above, we have:

$\left.\frac{d}{d t} I\left(u_{t}\right)\right|_{t=0}$

$=\int_{X} g_{M}\left(\nabla^{\mathrm{A}, \psi, *} D_{\mathrm{A}} u, d u(Y)\right) d v o l_{X}+\int_{X} g_{M} \otimes g_{X}\left(D_{\mathrm{A}} u, K_{\iota Y}^{M} F_{\mathrm{A}}\right) d v o l_{X}$

$+\frac{1}{24} \int_{X} \pi_{!}\left(\left(\iota_{Y} u^{*} \gamma_{0}\right) s_{X}\right) d v o l_{X}+\frac{1}{2} \int_{X} \pi_{!}\left(\operatorname{tr}\left(\left[\left(\iota_{Y} u^{*} \gamma_{1}\right), \mathcal{W}_{\varphi}^{+}\right] \wedge\left(\theta_{X} \wedge \theta_{X}^{T}\right)_{+}\right)\right) d v o l_{X}$ 


$$
-\frac{1}{2} \int_{X} \pi_{!}\left(\left\langle\left(\left\langle F_{\mathrm{B}}, \iota_{Y} d\left(\mu_{G} \circ u\right)\right\rangle\right) \wedge\left(\theta_{X} \wedge \theta_{X}^{T}\right)_{+}\right\rangle_{\mathfrak{s u}(2)}\right)
$$

Remark 4.8.3. Although the curve $t \longrightarrow u_{t}$ may not be $C^{1}$ in $W^{1,2}(Q, M)^{\operatorname{Spin}_{\varepsilon}^{G}(4)}$, the restriction of the energy functionals to the curve can be $C^{1}$ (c.f 4.7.6). In the above identity, if one can express the first the term in way so that the expression is valid for any $u \in W^{1,2}(Q, M)^{\operatorname{Spin}_{\varepsilon}^{G}(4)} \cong W^{1,2}\left(X, Q \times_{\operatorname{Spin}_{\varepsilon}^{G}(4)} M\right)$, then one may be able to derive the condition for a spinor $u \in W^{1,2}(Q, M)^{\operatorname{Spin}_{\varepsilon}^{G}(4)}$ to be a critical point of the energy functional $E(u, \mathrm{~A})$, analogous to the 4.7.6. Combining this condition with the energy identity above would be the first step in establishing a blow-up formula for "stationary" spinors. 


\title{
5. Generalized Seiberg-Witten equations
}

\author{
But thy eternal summer shall not fade, \\ Nor lose the possession of that fair thou owest. \\ - William Shakespeare, Sonnet 18
}

In this chapter, we introduce the generalized Seiberg-Witten equations associated to a hyperKähler manifold admitting a permuting action of $\operatorname{Spin}_{\varepsilon}^{G}(3)$. We discuss the particular case where $M$ is the $U(1)$-hyperKähler quotient of the flat space $\mathbb{H}^{n+1} \backslash\{0\}$ and obtain a priori estimates for the generalized spinors and the connection for the same.

\subsection{Generalized Seiberg-Witten Equations}

Having defined the generalized Dirac operator and the hyperKähler moment map in the previous chapters, we are now in a position to state the generalized SeibergWitten equations. Let $\left(X, g_{X}\right)$ be a four-dimensional, oriented, compact Riemann manifold. Let $M$ be a hyperKähler manifold admitting a permutting action of the $\operatorname{group} \operatorname{Spin}_{\varepsilon}^{G}(3)$ and a moment map $\mu: M \longrightarrow \mathfrak{s p}(1)^{*} \otimes \mathfrak{g}^{*}$.

Let B be a connection on the principal bundle $P_{G /\{\varepsilon\}} \longrightarrow X$. Let $\varphi$ be the LeviCivita connection on $X$. Let $\mathscr{A} \subset \mathscr{C}(Q)$ be the set of connections on $Q$ which are a lift of the Levi-Civita connection. Let $\mathrm{A} \in \mathscr{A}$ be the connection determined by B.

Denote by

$$
F_{\mathrm{A}} \in C^{\infty}\left(Q, \Lambda^{2}\left(\mathbb{R}^{4}\right) \otimes \mathfrak{s p i n}_{\varepsilon}^{G}(4)\right)^{\operatorname{Spin}_{\varepsilon}^{G}(4)}
$$

the full curvature of the connection $\mathrm{A}$ and $F_{\mathrm{B}}^{+} \in C^{\infty}\left(Q, \Lambda_{+}^{2}\left(\mathbb{R}^{4}\right) \otimes \mathfrak{g}\right)^{\operatorname{Spin}_{\varepsilon}^{G}(4)}$ the projection to the self-dual part of $\mathfrak{g}$-valued component of $F_{\mathrm{A}}$. 
Define the isomorphism

$$
\begin{aligned}
\delta: \Lambda_{+}^{2}\left(\mathbb{R}^{4}\right) \times \mathfrak{g} & \longrightarrow \mathfrak{s p}(1)^{*} \times \mathfrak{g}^{*} \\
\delta(\gamma)\left(\eta_{l} \otimes \eta\right) & =\left\langle\left\langle\gamma, \xi_{l}\right\rangle, \eta\right\rangle_{\mathfrak{g}}
\end{aligned}
$$

where $\gamma \in \Lambda_{+}^{2}\left(\mathbb{R}^{4}\right) \times \mathfrak{g}, \eta_{l}$ and $\xi_{l}$ are the basis elements of $\mathfrak{s p}(1)$ and $\Lambda_{+}^{2} \mathbb{R}^{4}$ respectively, $\eta \in \mathfrak{g}$ and $\langle\cdot, \cdot\rangle_{\mathfrak{g}}$ is the Ad-invariant inner product given by the negative of the Killing form. Supressing the isomorphism, the generalized Seiberg-Witten equations can be stated as:

$$
\left\{\begin{array}{l}
\not{P}_{\mathrm{A}} u=0 \\
F_{\mathrm{A}}^{+}-\mu \circ u=0
\end{array}\right.
$$

The Seiberg-Witten equations can be interpreted as a $\mathscr{G}=C^{\infty}(Q, G)^{\operatorname{Spin}_{\varepsilon}^{G}(4)_{-}}$ equivariant section of an infinite dimensional vector bundle. Indeed, recall that the generalized Dirac operator defines a $\mathscr{G}$-equivariant section of the vector bundle $\mathbb{W}^{-} \longrightarrow \mathrm{C}$, where $\mathrm{C}=\mathscr{S} \times \mathscr{Z}$ is the configuration space and $\mathbb{W}^{-}$is the infinite dimensional vector bundle over $\mathrm{C}$ with the fibre at a point $u \in \mathscr{S}$ being given by $C^{\infty}\left(Q, u^{*} \mathrm{~W}^{-}\right)$.

Let $\mathscr{N}$ denote the trivial vector bundle over the infinte dimensional manifold C with fibre $C^{\infty}\left(Q, \mathfrak{s p}(1)^{*} \otimes \mathfrak{g}^{*}\right)^{\operatorname{Spin}_{\varepsilon}^{G}(4)}$. Interpreting the second component of the equations as a section of $\mathscr{N}$, it is now clear that the Seiberg-Witten equations can

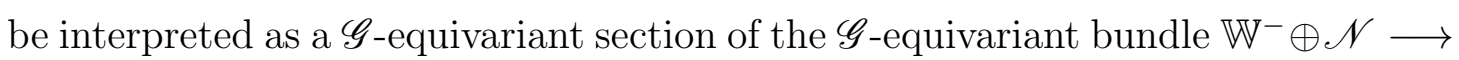
C, i.e:

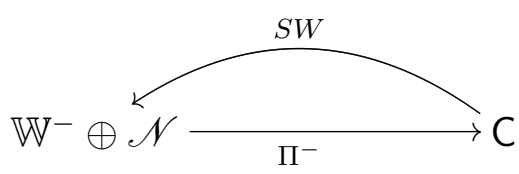

The solution of the equations are given by $S W^{-1}(0):=\mathscr{Z}\left(g_{X}\right)$. Supressing the dependence on the metric, we define the moduli space to be

$$
\mathfrak{M}=\mathscr{Z} / \mathscr{G}
$$




\subsection{Modified Seiberg-Witten equations}

We are interested in studying the Seiberg-Witten equations associated to the total space of Swann bundle over the Wolf space $X(n-1)$. The total space of the bundle is the higest weight nilpotent orbit of the group $S L(n+1, \mathbb{C})$ acting by adjoint action on its lie algebra $\mathfrak{s l}(n+1, \mathbb{C})$. Denote this total space by $\mathcal{O}_{n+1}$.

Consider the following scenario:

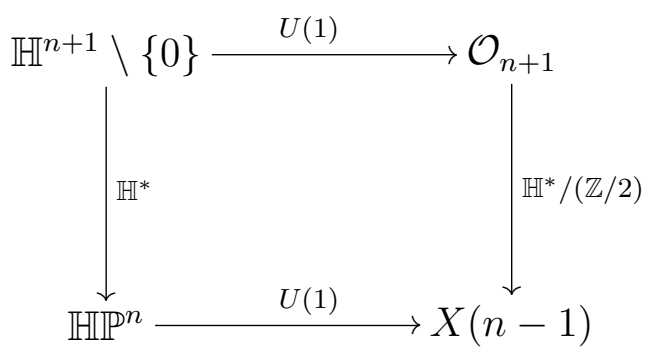

The group $U(1)$ acts hyperKähler on $\mathbb{H}^{n+1} \backslash\{0\}$, the action being given by

$$
\mathbb{H}^{n+1} \backslash\{0\} \ni h \mapsto e^{i \theta} h
$$

for $\theta \in[0,2 \pi)$. This action is free and descends to an action of $U(1)$ on $\mathbb{H} \mathbb{P}(n)$.

The moment map associated to the $U(1)$-action on $\mathbb{H}^{n+1} \backslash\{0\}$ is given by

$$
\mu: \mathbb{H}^{n+1} \backslash\{0\} \rightarrow \mathfrak{s p}(1)^{*}, \quad h \longmapsto \frac{1}{2} h i \bar{h} .
$$

The $U(1)$ action on $\mathbb{H}^{n+1}$ descends to an action on $\mathbb{H} \mathbb{P}(n)$. The moment map for the $U(1)$-action on $\mathbb{H} \mathbb{P}(n)$ is given by $\bar{\mu}([u])=\frac{1}{2} u i \bar{u}$. The zero-level set for $\bar{\mu}$ is given by $M_{0}=\{[h] \in \mathbb{H} \mathbb{P}(n) \mid h i \bar{h}=0\}$. This is a submanifold of $\mathbb{H} \mathbb{P}(n)$ and is invariant under the $U(1)$-action. One can prove that the group $U(n+1)$ acts transitively on $M_{0}$. The stabilizer of a point is given by $S U(2) \times U(n-1)$. Hence, the quaternionic Kähler quotient $M_{U(1)}:=M_{0} / U(1) \cong \frac{U(n+1)}{S U(2) \times U(n-1) \times U(1)}=\mathbb{G} r_{2}\left(C^{n+1}\right)$, the Grassmannian. This is the Wolf space $X(n-1)$.

Swann proves [Swa91] that the hyperKähler and quaternionic Kähler quotient constructions commutes with the construction of Swann bundles. This implies that $\mathcal{O}_{n+1}$ can be written as a $U(1)$-hyperKähler quotient of the flat space $\mathbb{H}^{n+1} \backslash\{0\}$. 
In addition to the $U(1)$-hyperKähler action, we also have a hyperKähler action of $S U(n)$, given by

$$
\mathbb{H}^{n+1} \backslash\{0\} \ni h \mapsto A h
$$

for $A \in S U(n)$. This action commutes with the $U(1)$ action and therefore we have a hyperKähler action of the group $S U(n) \times U(1)$ on $\mathbb{H}^{n+1} \backslash\{0\}$. For simplicity, we shall denote by $G:=S U(n) \times U(1)$.

The group $S p(1)$ has a permuting action on $\mathbb{H}^{n+1} \backslash\{0\}$, given by

$$
\mathbb{H}^{n+1} \backslash\{0\} \ni h \mapsto h \bar{q}
$$

where $q \in S p(1)$. Observe now that $S p(1)$ and the $G$-actions commute. The element $\{1,-1\} \in S p(1) \times G$ acts trivially on $\mathbb{H}^{n+1} \backslash\{0\}$, and hence the action descends to an action of $\operatorname{Sp}(1) \times_{ \pm 1} G \cong \operatorname{Spin}(3) \times{ }_{ \pm 1} G$.

Although we are interested in the Seiberg-Witten equations associated to the hyperKähler manifold $\mathcal{O}_{n+1}$, given the ease of analysis on $\mathbb{H}^{n+1} \backslash\{0\} \hookrightarrow \mathbb{H}^{n+1}$ compared to $\mathcal{O}_{n+1}$, we shall modify the generalized Seiberg-Witten equations slightly so as to use the equations associated to the former in order to construct the solutions for the latter one.

The modification is given by an additional condition $\mu_{U(1)} \circ u=0$ on the equations. More precisely, with slight abuse of notation, let $u \in C^{\infty}\left(Q, \mathbb{H}^{n+1}\right)^{\operatorname{Spin}_{\varepsilon}^{G}(4)}$. Then the modified Seiberg-Witten equations are:

$$
\left\{\begin{array}{l}
\mathscr{D}_{\mathrm{A}} u=0 \\
F_{\mathrm{B}_{S U(n+1)}}^{+}-\mu_{S U(n)} \circ u=0 \\
\mu_{U(1)} \circ u=0
\end{array}\right.
$$

Here, $\mathrm{B}_{S U(n+1)}$ denotes the $\mathfrak{s u}(n)$-component of the connection $\mathrm{B}_{G}=\mathrm{B}_{U(1)} \oplus \mathrm{B}_{S U(n+1)}$, and $\mu_{S U(n)}$ and $\mu_{U(1)}$ denote the moment maps for the $S U(n)$ and the $U(1)$ action respectively.

Denote by $\rho_{+}: \operatorname{Spin}(4) \times_{ \pm 1} G \longrightarrow \operatorname{Spin}(3) \times_{ \pm 1} G$, the homomorphism $\rho_{+}\left(\left[q_{+}, q_{-}, g\right]\right)=\left[q_{+}, g\right]$, and now consider the trivial vector bundle $\mathbb{H}^{n+1}$ to be a $\operatorname{Spin}(4) \times_{ \pm 1} G$-equivariant vector bundle with the action induced by $\rho_{+}$. We shall henceforth denote the $\operatorname{Spin}_{\varepsilon}^{G}(4)$-space $\mathbb{H}^{n+1}$, with the mentioned action, by $\mathrm{W}^{+}$. We define the $\operatorname{Spin}_{\varepsilon}^{G}(4)$-equivariant vector bundle $\mathbf{W}^{-}$to be $\mathbb{H}^{n+1}$ equipped with the action $\left(\left[q_{+}, q_{-}, g\right]\right) h_{-}=q_{-} g h_{-}$, where $h_{-} \in \mathbb{H}^{n+1}$. 
In order to work out the further analysis, we need appropriate sobolev completions of the infinite-dimensional manifolds $\mathscr{S}, \mathscr{A}$. Fix a smooth connection $A \in \mathscr{A}$. Then for $k \in \mathbb{N}$ and $1 \leq p<\infty$, define

$$
\mathscr{S}^{k, p}:=W^{k, p}\left(Q, \mathbb{H}^{n+1}\right)^{\operatorname{Spin}_{\varepsilon}^{G}(4)} \cong W^{k, p}\left(X, Q \times_{\operatorname{Spin}_{\varepsilon}^{G}(4)} \mathbb{H}^{n+1}\right)
$$

to be the completion of $\mathscr{S}=C^{\infty}\left(Q, \mathbb{H}^{n+1}\right)^{\operatorname{Spin}_{\varepsilon}^{G}(4)} \cong \Gamma\left(X, Q \times_{\operatorname{Spin}_{\varepsilon}^{G}(4)} \mathbb{H}^{n+1}\right)$ w.r.t the norm

$$
\|u\|_{k, p}=\left(\sum_{i=1}^{k} \int_{X}\left\|\left(D_{\mathrm{A}}^{i}\right) u\right\|^{\frac{p}{2}}\right)^{\frac{1}{p}} .
$$

Additionally, assume that $k-\frac{4}{p}>0$ so that the Sobolev embedding $W^{k, p} \hookrightarrow C^{0}$ is continuous.

\subsection{Variational Formulation}

For $G=U(1) \times S U(n)$ and any generalized spinor $u \in \mathscr{S}$, the Weitzenböck formula (4.5.1) reads:

$$
\mathfrak{D}_{\mathrm{A}}^{*} \mathfrak{D}_{\mathrm{A}} u=D_{\mathrm{A}}^{*} D_{\mathrm{A}} u+\frac{1}{4} s_{X} u+\mathfrak{c}\left(F_{\mathrm{B}_{S U(n+1)}}^{+}\right) u+\mathfrak{c}\left(F_{\mathrm{B}_{U(1)}}^{+}\right) u .
$$

Correspondingly we have:

$$
\begin{aligned}
\left\|\mathscr{P}_{\mathrm{A}} u\right\|_{L^{2}}^{2} & =\left\|D_{\mathrm{A}} u\right\|_{L^{2}}^{2}+\frac{1}{4} \int_{X} s_{X}\|u\|^{2}+2 \int_{X}\left\langle\mu_{S U(n)} \circ u, F_{\mathrm{B}_{S U(n+1)}}^{+}\right\rangle \operatorname{vol}_{X} \\
& +2 \int_{X}\left\langle\mu_{U(1)} \circ u, F_{\mathrm{B}_{U(1)}^{+}}^{+}\right\rangle \operatorname{vol}_{X} .
\end{aligned}
$$

The energy functional for the modified Seiberg-Witten equations is given by:

$$
\mathcal{E}(u, \mathrm{~A})=\left\|\mathscr{D}_{\mathrm{A}} u\right\|_{L^{2}}^{2}+\left\|F_{\mathrm{B}_{S U(n+1)}}^{+}-\mu_{S U(n)} \circ u\right\|_{L^{2}}^{2}+\left\|\mu_{U(1)} \circ u\right\|_{L^{2}}^{2} .
$$

Its, of course, obvious that the solutions to (5.1.1) are absolute minimizers of $\mathcal{E}$.

Using (5.3.2), we can re-write the energy functional as:

$$
\begin{aligned}
\mathcal{E}(u, \mathrm{~A}) & =\left\|D_{\mathrm{A}} u\right\|_{L^{2}}^{2}+\left\|F_{\mathrm{B}_{S U(n+1)}}^{+}\right\|_{L^{2}}^{2}+\left\|\mu_{S U(n)} \circ u\right\|_{L^{2}}^{2}+\frac{1}{4} s_{X}\|u\|_{L^{2}}^{2} \\
& +\left\|\mu_{U(1)} \circ u\right\|_{L^{2}}^{2}+2 \int_{X}\left\langle\mu_{U(1)} \circ u, F_{\mathrm{B}_{U(1)}}^{+}\right\rangle \operatorname{vol}_{X} .
\end{aligned}
$$


Recall from the previous chapter that the moment map for the hyperKähler action of $S U(n) \curvearrowright \mathbb{H}^{n} \backslash\{0\}$ is given by:

$$
\begin{gathered}
\mu_{S U(n)}: \mathbb{H}^{n} \cong \mathbb{H} \otimes_{\mathbb{C}} \mathbb{C}^{n} \longrightarrow \mathfrak{s p}(1) \otimes \mathfrak{s u}(n) \\
\mu_{S U(n)}(h \otimes z)=\frac{1}{4 n} h i \bar{h} \otimes\left(i z z^{*}-i\|z\|^{2} \cdot i d_{\mathbb{C}^{n}}\right)
\end{gathered}
$$

We therefore have:

$$
\begin{aligned}
\left\|\mu_{S U(n)}(h \otimes z)\right\|^{2} & =\frac{1}{4 n}\left\|h i \bar{h} \otimes\left(i \bar{z} z^{t}-i\|z\|^{2} \cdot i d_{\mathbb{C}^{n}}\right)\right\|^{2} \\
& =\frac{1}{4 n}\|h\|^{4}\left(-2 n \operatorname{tr}\left(\left(i \bar{z} z^{t}-i\|z\|^{2} \cdot i d_{\mathbb{C}^{n}}\right)\left(i \bar{z} z^{t}-i\|z\|^{2} \cdot i d_{\mathbb{C}^{n}}\right)\right)\right) \\
& =\frac{1}{4 n}\|h\|^{4}\left(-2 n \operatorname{tr}\left(i \bar{z} z^{t} i \bar{z} z^{t}-2 i z z^{*} i\|z\|^{2} \cdot i d_{\mathbb{C}^{n}}+i\|z\|^{2} i\|z\|^{2} i d_{\mathbb{C}^{n}}\right)\right) \\
& =\frac{1}{4 n}\|h\|^{4}\left(2 n \operatorname{tr}\left(\|z\|^{2} \bar{z} z^{t}-2\|z\|^{2} \bar{z} z^{t}+\|z\|^{4} i d_{\mathbb{C}^{n}}\right)\right) \\
& =\frac{1}{4 n}\|h\|^{4}\left(2 n\left(\|z\|^{4}-2\|z\|^{4}+n\|z\|^{4}\right)\right) \\
& =\frac{1}{4 n}\|h\|^{4}\left(2 n(n-1)\|z\|^{4}\right) \\
& =\frac{(n-1)}{2}\|h\|^{4}\|z\|^{4}
\end{aligned}
$$

This implies that

$$
\left.\left\|\mu_{S U(n)} \circ u\right\|^{2}\right|_{q}=\left.\frac{(n-1)}{2}\|u\|^{4}\right|_{q} .
$$

Also, the moment map for the $U(1)$ hyperKähler action is given by

$$
\mu_{U(1)}(h \otimes z)=\frac{1}{2} h i \bar{h} \otimes i \cdot\|z\|^{2},
$$

and so

$$
\left.\left\|\mu_{U(1)} \circ u\right\|^{2}\right|_{q}=\left.\frac{1}{2}\|u\|^{4}\right|_{q}
$$

Therefore from (5.3.4) and (5.3.5), we get:

$$
\begin{aligned}
\mathcal{E}(u, \mathrm{~A}) & =\left\|D_{\mathrm{A}} u\right\|_{L^{2}}^{2}+\left\|F_{\mathrm{B}_{S U(n+1)}^{+}}^{+}\right\|_{L^{2}}^{2}+\frac{1}{4} s_{X}\|u\|_{L^{2}}^{2}+C \cdot\|u\|_{L^{2}}^{2} \\
& +2 \int_{X}\left\langle\mu_{U(1)} \circ u, F_{\mathrm{B}_{U(1)}}^{+}\right\rangle \operatorname{vol}_{X},
\end{aligned}
$$


where $C$ is the constant given by $C=\frac{n}{2}$. It is clear that any solution to generalized Seiberg-Witten equations is an absolute minimizer of the energy-functional $\mathcal{E}$. Observe that using the identity

$$
\left\|F_{A}\right\|^{2}=-\operatorname{tr} \int_{X} F_{A} \wedge * F_{A}=-\operatorname{tr} \int_{X} F_{A}^{+} \wedge * F_{A}^{+}-\operatorname{tr} \int_{X} F_{A}^{-} \wedge * F_{A}^{-}=\left\|F_{A}^{+}\right\|^{2}+\left\|F_{A}^{+}\right\|^{2},
$$

we have

$$
\begin{aligned}
\left\|F_{\mathrm{B}_{S U(n+1)}}^{+}\right\|^{2} & =\frac{1}{2}\left\|F_{\mathrm{B}_{S U(n+1)}}\right\|^{2}+\left\|F_{\mathrm{B}_{S U(n+1)}}^{+}\right\|^{2}-\frac{1}{2}\left\|F_{\mathrm{B}_{S U(n+1)}}\right\|^{2} \\
& =\frac{1}{2}\left\|F_{\mathrm{B}_{S U(n+1)}}\right\|^{2}+\frac{1}{2}\left(\left\|F_{\mathrm{B}_{S U(n+1)}^{+}}^{+}\right\|^{2}+\left\|F_{\mathrm{B}_{S U(n+1)}}\right\|^{2}\right) \\
& =\frac{1}{2}\left\|F_{\mathrm{B}_{S U(n+1)}}\right\|^{2}-\frac{1}{2} \operatorname{tr} \int_{X} F_{A} \wedge * F_{A}
\end{aligned}
$$

Since the second term is the second chern-number of the underlying principal bundle, a constant, we can instead consider the energy functional:

$$
\begin{aligned}
\mathcal{E}^{\prime}(u, \mathrm{~A}) & =\left\|D_{\mathrm{A}} u\right\|_{L^{2}}^{2}+\left\|F_{\mathrm{B}_{S U(n+1)}}\right\|_{L^{2}}^{2}+\frac{1}{4} \int_{X} s_{X}\|u\|^{2}+C \cdot\|u\|_{L^{2}}^{2} \\
& +2 \int_{X}\left\langle\mu_{U(1)} \circ u, F_{\mathrm{B}_{U(1)}}\right\rangle \operatorname{vol}_{X},
\end{aligned}
$$

The last term is a consequence of the identification $\mathfrak{s p}(1)^{*} \cong \Lambda_{+}^{2} \mathbb{R}^{*}$. The EulerLagrange equations for $\mathcal{E}$ and $\mathcal{E}^{\prime}$ are the same.

Lemma 5.3.1. The Euler-Lagrange Equations for $\mathcal{E}(u, \mathrm{~A})$ are given by

$$
\left\{\begin{array}{l}
D_{\mathrm{A}}^{*} D_{\mathrm{A}} u+s_{X} u+C u+2 \mathfrak{c}\left(F_{\mathrm{B}_{U(1)}}\right) u=0 \\
d_{\mathrm{A}}^{*} F_{\mathrm{A}}^{+}+(d \mu(i))^{*} \mathrm{I}_{1}\left(D_{\mathrm{A}} u\right)+d_{\mathrm{A}}^{*}\left(\mu_{U(1)} \circ u\right)=0
\end{array}\right.
$$

Proof : Let $u_{t} \in \mathscr{S}$ be a smooth curve and let $\left.\frac{d}{d t} u_{t}\right|_{t=0}=u^{\prime}$. Then

$$
\begin{aligned}
\mathcal{E}^{\prime}\left(u_{t}, \mathrm{~A}\right) & =\left\|D_{\mathrm{A}} u_{t}\right\|_{L^{2}}^{2}+\left\|F_{\mathrm{B}_{S U(n+1)}}\right\|_{L^{2}}^{2}+\frac{1}{4} s_{X}\left\|u_{t}\right\|_{L^{2}}^{2}+C \cdot\left\|u_{t}\right\|_{L^{2}}^{2} \\
& +2 \int_{X}\left\langle\mu_{U(1)} \circ u_{t}, F_{\mathrm{B}_{U(1)}}\right\rangle \operatorname{vol}_{X},
\end{aligned}
$$

We have,

$$
\left.\frac{d}{d t}\left\|D_{\mathrm{A}} u_{t}\right\|^{2}\right|_{t=0}=2 \int_{X}\left\langle D_{\mathrm{A}}^{*} D_{\mathrm{A}} u, u^{\prime}\right\rangle d v o l_{X}
$$




$$
\begin{gathered}
\left.\frac{d}{d t} \int_{X} s_{X}\left\|u_{t}\right\|^{2} d v o l_{X}\right|_{t=0}=2 \int_{X} s_{X}\left\langle u, u^{\prime}\right\rangle d v o l_{X}, \\
\left.\frac{d}{d t} \int_{X} C\left\|u_{t}\right\|^{2}\right|_{t=0} d v o l_{X}=2 \int_{X} C\left\langle u, u^{\prime}\right\rangle, \\
\left.\left.\frac{d}{d t} \int_{X}\left\langle\mu_{U(1)} \circ u_{t}, F_{\mathrm{B}_{U(1)}}\right\rangle d v o l_{X}\right|_{t=0}=\int_{X} \frac{d}{d t} \mathfrak{c}\left(F_{\mathrm{B}_{U(1)}}\right) u_{t}, u_{t}\right\rangle\left.d v o l_{X}\right|_{t=0}=4 \int_{X}\left\langle\mathfrak{c}\left(F_{\mathrm{B}_{U(1)}}\right) u, u^{\prime}\right\rangle d v o l_{X} .
\end{gathered}
$$

Therefore $\left.\frac{d}{d t} \mathcal{E}\left(u_{t}, \mathrm{~A}\right)\right|_{t=0}=0$ gives the first equation:

$$
D_{\mathrm{A}}^{*} D_{\mathrm{A}} u+s_{X} u+C u+2 \mathfrak{c}\left(F_{\mathrm{B}_{U(1)}}\right) u=0
$$

Define I $: \mathfrak{s p}(1) \longrightarrow \mathbb{H}^{n+1}$ by $\mathrm{I}(\xi)(p)=p \cdot \bar{\xi}$ for $p \in \mathbb{H}^{n+1}$. Let $\xi \in \mathfrak{s p}(1)$ such that $\xi^{2}=-1$ and $Y \in T_{x} \mathbb{H}^{n+1}$. Then,

$$
\begin{aligned}
\langle\eta \cdot x, Y\rangle_{\mathbb{H}^{n+1}} & =\langle\eta \cdot x \cdot \bar{\xi}, Y \cdot \bar{\xi}\rangle_{\mathbb{H}^{n+1}}=\omega(\xi)(\eta \cdot x, \mathrm{I}(\xi)(Y)) \\
& =d \mu_{G}(\xi \otimes \eta)(\mathrm{I}(\xi)(Y))=\left\langle\eta,\left(d \mu_{G}(\xi)\right)^{*}(\mathrm{I}(\xi)(Y))\right\rangle_{\mathfrak{g}}
\end{aligned}
$$

Since this is true for any $\xi \in \mathfrak{s p}(1)$, such that $\xi^{2}=-1$, choose $\xi=i$ and let $\mathrm{I}_{1}=\mathrm{I}(i)$. Therefore, we get

$$
\langle\eta \cdot x, Y\rangle_{\mathbb{H}^{n+1}}=d \mu_{G}(\xi \otimes \eta)\left(\mathrm{I}_{1}(Y)\right)=\left\langle\eta,\left(d \mu_{G}(i)\right)^{*}\left(\mathrm{I}_{1}(Y)\right)\right\rangle_{\mathfrak{g}} .
$$

Let $\mathrm{A}_{t}=\mathrm{A}+t \alpha$. We have:

$$
\begin{aligned}
\mathcal{E}\left(u, \mathrm{~A}_{t}\right) & =\left\|D_{\mathrm{A}_{t}} u\right\|_{L^{2}}^{2}+\left\|F_{\mathrm{B}_{S U(n+1)}^{t}}\right\|_{L^{2}}^{2}+\frac{1}{4} s_{X}\|u\|_{L^{2}}^{2}+C \cdot\|u\|_{L^{2}}^{2} \\
& +2 \int_{X}\left\langle\mu_{U(1)} \circ u, F_{\mathrm{B}_{U(1)}^{t}}\right\rangle \operatorname{vol}_{X},
\end{aligned}
$$

where $\mathrm{B}_{S U(n+1)}^{t}$ and $\mathrm{B}_{U(1)}^{t}$ are the $\mathfrak{s u}(n+1)$ and $\mathfrak{u}(1)$-components respectively, of the full connection $A_{t}$. Therefore,

$$
\begin{aligned}
\left.\frac{d}{d t}\left\langle D_{\mathrm{A}_{t}} u, D_{\mathrm{A}_{t}} u\right\rangle_{\mathbb{H}^{n+1}}\right|_{t=0} & =\left.2\left\langle D_{\mathrm{A}} u, \frac{d}{d t} D_{\mathrm{A}_{t}} u\right\rangle_{\mathbb{H}^{n+1}}\right|_{t=0}=2\left\langle D_{\mathrm{A}} u, \alpha \cdot u\right\rangle_{\mathbb{H}^{n+1}} \\
& =2\left\langle\left(d \mu_{G}(i)\right)^{*} \mathrm{I}_{1}\left(D_{\mathrm{A}} u\right), \alpha\right\rangle_{T^{*} X \otimes \mathfrak{g}}
\end{aligned}
$$

The last expression follows from 5.3.10. Computations, as in the case of Yang-Mills functional, give

$$
\left.\frac{d}{d t}\left\langle F_{\mathrm{B}_{S U(n+1)}^{t}}, F_{\mathrm{B}_{S U(n+1)}^{t}}\right\rangle\right|_{t=0}=2\left\langle d_{\mathrm{B}_{S U(n+1)}^{*}}^{*} F_{\mathrm{B}_{S U(n+1)}}, \alpha_{S U(n+1)}\right\rangle
$$


and also

$$
2 \frac{d}{d t} \int_{X}\left\langle\left(\mu_{U(1)} \circ u\right), F_{\mathrm{B}_{U(1)}^{t}}\right\rangle \operatorname{vol}_{X}=2 \int_{X}\left\langle d_{\mathrm{A}}^{*}\left(\mu_{U(1)} \circ u\right), \alpha_{U(1)}\right\rangle \operatorname{vol}_{X} .
$$

It follows from the above computations that

$$
\frac{d}{d t} \mathcal{E}^{\prime}\left(u, \mathrm{~A}_{t}\right)=0 \Longrightarrow d_{\mathrm{A}}^{*} F_{\mathrm{A}}+\left(d \mu_{G}(i)\right)^{*} \mathrm{I}_{1}\left(D_{\mathrm{A}} u\right)+d_{\mathrm{A}}^{*}\left(\mu_{U(1)} \circ u\right)=0 .
$$

\subsection{A priori estimates}

For the generalized Seiberg-Witten equations, Henrik Schumacher [Sch10] derived sufficient conditions for the existence of the $L^{\infty}$-estimates on the spinor part of the solution. For the case $M=\mathbb{H}^{n+1}$, these are automatically satisfied.

Proposition 5.4.1. Let $X$ compact Riemann four-manifold with a $\operatorname{Spin}_{\varepsilon}^{G}(4)$ structure $Q \stackrel{\pi_{Q}}{\longrightarrow} X$. Let $(u, \mathrm{~A})$ be any solution to the modified Seiberg-Witten equations above and let $s_{X}$ denote the scalar curvature function on $X$, Denote by $s_{X}^{-}=\max \left\{0,-\frac{1}{4} \min _{x \in X} s_{X}(x)\right\}$. Then the following estimates hold:

1. $\left.\|u\|^{2}\right|_{q} \leq s_{X}^{-}$

2. $\left\|D_{\mathrm{A}} u\right\|_{L^{2}}^{2} \leq \frac{1}{8} \int_{X}\left(s_{X}\right)^{2} \operatorname{vol}_{X}$

3. $\left\|F_{\mathrm{B}_{S U(n+1)}}^{+}\right\| \|_{q} \leq \frac{1}{2} n s_{X}^{-}$

\section{Proof :}

1. Let $q \in Q$ and $\pi_{Q}(q)=x \in X$. In a neighbourhood of $x$, choose a local orthonormal frame $\left\{X_{l}\right\}$ such that $\left\{e_{i}\right\} \in \mathbb{R}^{4}$ at $x \in \pi_{Q}(q)$ such that $\left.X_{l}\right|_{\pi_{Q}(q)}=\pi_{Q}(q)\left(e_{l}\right)$ and $\left.\nabla_{X_{i}} X_{j}\right|_{\pi_{Q}(q)}=0$ and denote by $Y_{l}=\tilde{X}_{l}$ the horizontal lift to $T Q$ by the connection $A$. Note that the function $\|u\|^{2}$ is $\operatorname{Spin}_{\varepsilon}^{G}(n)$ invariant and therefore descends to a function $f$ on the base manifold $X$. Let $\nabla$ denote the covariant derivative w.r.t the Levi-Civita connection. 
We shall now compute $\triangle_{X} f$ :

$$
\begin{aligned}
\triangle_{X} f(x) & =-\left.\sum_{i=1}^{4} \nabla_{X_{l}} \nabla_{X_{l}} f\right|_{x} \\
& =-\left.\sum_{i=1}^{4} D_{\mathrm{A}, Y_{l}} D_{\mathrm{A}, Y_{l}}\|u\|^{2}\right|_{q}=-\left.\sum_{i=1}^{4} D_{\mathrm{A}, Y_{l}} D_{\mathrm{A}, Y_{l}}\langle u, u\rangle\right|_{q} \\
& =-\sum_{i=1}^{4} D_{\mathrm{A}, Y_{l}}\left(\left.\left\langle D_{\mathrm{A}, Y_{l}} u, u\right\rangle_{\mathbb{H}^{n}}\right|_{q}+\left.\left\langle u, D_{\mathrm{A}, Y_{l}} u\right\rangle_{\mathbb{H}_{n}}\right|_{q}\right. \\
& =-\left.\sum_{i=1}^{4} D_{\mathrm{A}, Y_{l}}\left\langle D_{\mathrm{A}, Y_{l}} u, u\right\rangle_{\mathbb{H}^{n}}\right|_{q}+\left.D_{\mathrm{A}, Y_{l}}\left\langle D_{\mathrm{A}, Y_{l}} u, u\right\rangle_{\mathbb{H}^{n}}\right|_{q} \\
& =-\left.2 \sum_{i=1}^{4}\left\langle D_{\mathrm{A}, Y_{l}} D_{\mathrm{A}, Y_{l}} u, u\right\rangle_{\mathbb{H}^{n}}\right|_{q}-\left.2 \sum_{i=1}^{4}\left\|D_{\mathrm{A}, Y_{l}} u\right\|^{2}\right|_{q}
\end{aligned}
$$

This gives $\triangle_{X} f(x) \leq\left. 2\left\langle D_{\mathrm{A}, Y_{l}}^{*} D_{\mathrm{A}, Y_{l}} u, u\right\rangle_{\mathbb{H}^{n}}\right|_{q}$.

Weitzenböck formula gives :

$$
\triangle_{X} f(x) \leq-\left.\frac{1}{2} s_{X}(x)\|u\|^{2}\right|_{q}-\left.4\left\langle\mu_{S U(n)} \circ u, F_{\mathrm{B}_{S U(n+1)}}^{+}\right\rangle\right|_{q} .
$$

At a point $x_{0}$ where $f$ attains a maximum, $\triangle_{X} f\left(x_{0}\right) \geq 0$ and hence we get

$$
0 \leq-\frac{1}{2} s_{X}\left(x_{0}\right)\left\|u\left(q_{0}\right)\right\|^{2}-\left.4\left\langle\mu_{S U(n)} \circ u, F_{\mathrm{B}_{S U(n+1)}}^{+}\right\rangle\right|_{q_{0}}
$$

where $\pi_{Q}\left(q_{0}\right)=x_{0}$.

Since $u$ is a solution to the Seiberg-Witten equations, $F_{\mathrm{B}_{S U(n+1)}^{+}}^{+}=\mu_{S U(n)} \circ u$ and so:

$$
\left.\left\|\mu_{S U(n)} \circ u\right\|^{2}\right|_{q_{0}} \leq-\frac{1}{8} s_{X}\left(x_{0}\right)\left\|u\left(q_{0}\right)\right\|^{2} .
$$

Therefore from (5.4.1) and (5.3.4) we get

$$
\left.\|u\|^{2}\right|_{q_{0}} \leq-\frac{1}{4} s_{X}\left(x_{0}\right) .
$$

Then $\left.\|u\|^{2}\right|_{q} \leq s_{X}^{-} \quad \forall q \in Q$.

This proves the claim (1). 
2. Since $(\mathrm{A}, u)$ is a solution to the modified Seiberg-Witten equations, $\mu_{U(1)} \circ u=0$. Therefore the Weitzenböck formula 5.3.1 implies:

$$
\begin{aligned}
0 & =\int_{X}\left\|D_{\mathrm{A}} u\right\|^{2} d v o l_{X}+\frac{1}{4} \int_{X} s_{X}\|u\|^{2} d v o l_{X}+2 \int_{X}\left\|\mu_{S U(n)} \circ u\right\|^{2} d v o l_{X} \\
& \geq \int_{X}\left\|D_{\mathrm{A}} u\right\|^{2} d v o l_{X}+\frac{1}{4} \int_{X} s_{X}\|u\|^{2} d v o l_{X}+\int_{X}\|u\|^{4} d v o l_{X}
\end{aligned}
$$

This implies:

$$
\int_{X}\left\|D_{\mathrm{A}} u\right\|^{2} d \operatorname{vol}_{X} \leq-\left[\int_{X}\left(\frac{1}{4} s_{X}\|u\|^{2}+\|u\|^{4}\right) d v o l_{X}\right] .
$$

Completing the term $\frac{1}{4} s_{X}\|u\|^{2}+\|u\|^{4}$ to a square, gives the estimate:

$$
0 \leq \int_{X}\left(\frac{1}{4} s_{X}\|u\|^{2}+\|u\|^{4}+\frac{1}{8} s_{X}^{2}\right) d v o l_{X}
$$

Therefore we have:

$$
\int_{X}\left\|D_{\mathrm{A}} u\right\|^{2} d v o l_{X}=-\int_{X}\left(\frac{1}{4} s_{X}\|u\|^{2}+\|u\|^{4}\right) d v o l_{X} \leq \frac{1}{8} \int_{X} s_{X}^{2} d v o l_{X} .
$$

This proves the claim (2).

3. Observe that, since $u$ is a solution to the modified SW equations from, claim

(1) we get

$$
\left.\left\|F_{\mathrm{B}_{S U(n+1)}}^{+}\right\|\right|_{q}=\left.\left\|\mu_{S U(n)} \circ u\right\|\right|_{q} \leq\left.\frac{1}{2} n\|u\|^{2}\right|_{q} \leq-\frac{1}{8} n s_{X}\left(x_{0}\right) \leq \frac{1}{2} n s_{X}^{-} .
$$

This proves claim (3). 


\section{Conclusion}

Now, here in this fort of Brahman, there is a small lotus, a dwelling-place, and within it, a small space. In that space there is something - and that's what you should try to discover, that's what you should seek to perceive."

- Chāndogya Upanishad, 4.8.1

The Seiberg-Witten equations analysed in Chapter 5 serve as model for a more general scenario. Many interesting hyperKähler manifolds in applications, like Gibbon-Hawking spaces [GS78], the moduli space of instantons over $\mathbb{R}^{4}$, etc. possess a hyperKähler structure, obtained via hyperKähler reduction of flat-spaces. For the model we have considered, we have the a priori, uniform $W^{1,2}$ bounds on the spinor and the $S U(n+1)$-component of the connection. But the $U(1)$-connection component, a priori, admits no such bounds. Indeed there exists canonical connection $\mathrm{A}_{c a n}:=\frac{1}{\rho_{0}}\langle h, \cdot\rangle_{\mathbb{H}^{n+1}}$ on $U(1)$-principal bundle $\mu_{U(1)}^{-1}(0) \longrightarrow \mathcal{O}_{n+1}$ [Hay08]. If the spinor is harmonic and $\mu_{U(1)} \circ u=0$, the $U(1)$-component of the connection is given by $u^{*} \mathrm{~A}_{c a n}=\frac{1}{\|u\|^{2}}\langle u, T u(\cdot)\rangle_{\mathbb{H}^{n+1}}$. Clearly, since the spinor is not bounded from below, the $U(1)$-connection component, a priori, is not under control.

As was shown in Section 4.8, harmonic spinors can be interpreted as a "gaugedversion" of the usual aholomorphic maps. Indeed, from Remarks 4.8.2 \& 4.8.1, it follows that in the case when the base manifold is hyperkähler, much of the properties of the usual aholomorphic maps are retained. In the light of this comparison, it is natural to explore the analogy with the known case of singularities of aholomorphic maps.

Let $M$ and $N$ be two hyperKähler manifolds with dimensions $m$ and $n$ respectively. Let $u_{k}: M \longrightarrow N$ be a sequence of aholomorphic maps such that $E\left(u_{k}\right) \leq C$. The blow-up set $\Sigma$ is of Hausdorff dimension at most $m-3$ and hence is stationary (cf. Remark 4.7.1).

Theorem 6.0.1. [LT98] Let $M$ and $N$ be as above. Then for a sequence of aholo- 
morphic maps with $E\left(u_{k}\right) \leq C$, such that $u_{k} \rightarrow u$ in $W^{1,2}(M, N)$, the limit map $u$ is a stationary harmonic map and is a smooth aholomorphic map on $M \backslash \Sigma$.

The obstruction to the strong convergence of the the sequence $u_{k}$ can be attributed to the existence of holomorphic spheres in the target manifold:

Theorem 6.0.2. [CLOO] Let $M$ be a compact hyperKähler surface and $N$ be as above and let $u_{k}$ be a sequence of aholomorphic maps with bounded energies. If $N$ does not admit a holomorphic $\mathbb{S}^{2}$ w.r.t the any complex structure on $\mathbb{S}^{2}$, induced by the two-sphere of complex structures on $\mathbb{R}^{4}$ and any complex structure in the two-sphere of complex structures on $N$, then the sequence $u_{k}$ converges strongly to a map $u \in W^{1,2}(M, N)$, which is smooth and aholomorphic on $M \backslash \Sigma$.

Although the harmonic spinors represent a generalized case of usual aholomorphic maps, the energy identities satisfied by both are significantly different. The main difficulty is that for a fixed smooth connection $\mathrm{A}$ on $Q$, and a generalized spinor $u$, the energy $E_{\tau}(u, \mathrm{~A})$ is not topological, as against the case of usual aholomorphic maps. Therefore the harmonic spinors need not be energy minimizers of $E(u, \mathrm{~A})$, as a consequence of which we cannot directly carry over the arguments from the aholomorphic case. From remarks $4.8 .1 \& 4.8 .2$, we see that when the base manifold is hyperKähler, $E_{\tau}(u, \mathrm{~A})$ is topological.

A natural question to ask is what is the minimum restriction on the base manifold for which the energy $E_{\tau}(u, \mathrm{~A})$ is invariant under smooth deformations of the source manifold? For instance we may argue that if the base manifold is locally hyperKähler (i.e, the restricted holonomy group is contained in $S p(1)$ ), then locally, the spinors are equivariant lifts of usual aholophormphic maps and the analogue of Theorem 4.7.1 holds for the spinors. Fix a smooth connection A. Then, for a sequence of harmonic spinors in $W^{1,2}(Q, M)^{\operatorname{Spin}_{\varepsilon}^{G}(4)} \cong W^{1,2}\left(X, Q \times_{\operatorname{Spin}_{\varepsilon}^{G}(4)} M\right)$, with bounded energies, converging weakly to $u$, we may define the singular set as $u$ as

$$
\Sigma(\mathrm{A})=\bigcap_{r>0}\left\{x \in X \mid \liminf _{k \rightarrow 0} r^{2-m} \int_{B_{r}(x)}\left\|D_{\mathrm{A}} u_{k}\right\|^{2} d y \geq \varepsilon_{0}\right\} .
$$

Using the identity 4.8.2, one may obtain a blow-up formula, analogous to Eq. 4.7.5, for harmonic spinors (c.f Remark 4.8.3). In this regard, the following question that arises is: Is the presence of holomorphic $\mathbb{S}^{2}$ in $M$, an obstruction to the locally strong convergence? Is it also the global obstruction? 


\section{Bibliography}

[ABS64] M. Atiyah, R. Bott, and A. Shapiro, Clifford Modules, Topology 3 (1964), 3-38.

[Ale68] D. V. Alekseevskii, Compact Quaternion Spaces, Funktsional'nyi Analiz i Ego Prilozheniya 2 (1968), no. 2, 11-20.

[Bau09] Helga Baum, Eichfeldtheorie. eine einführung in die differentialgeometrie auf faserbündeln, Springer-Verlag, Berlin, 2009.

[Bet93] Fabrice Bethuel, On the Singular set of Stationary harmonic maps, Manuscripta Mathematica 78 (1993), no. 1, 417-443.

[BGM93] C. P. Boyer, K. Galicki, and B. M. Mann, Quaternionic reduction and Einstein manifolds, Comm. Anal. Geom. (1993), no. 2, 229-279.

[BGM94] _ _ Quaternionic Geometry and 3-Sasakian Manifolds, Proceedings of the meeting on Quaternionic Structures in Mathematics and Physics, Treiste, ILAS/FM-6 (1996), SISSA, 1994.

[BL86] Brezis and Lieb, Harmonic Maps with Defects, Communications in Mathematical Physics 107 (1986), 649-705.

[Cal10] Martin Callies, Dimensional Reduction for the Generalized SeibergWitten equations and the Chern-Simons-Dirac functional, Master's thesis, Mathematisches Institut, Georg-August-Universität, Göttingen, http://www . uni-math.gwdg.de/preprint/mg. 2010.03.pdf, 2010.

[Che99] Jingyi Chen, Complex Anti-Self-Dual Connections on a Product of Calabi-Yau Surfaces and Triholomorphic Curves, Communications in Mathematical Physics 201 (1999), 217-247. 
[CL00] Jingyi Chen and Jiayu Li, Quaternionic maps between Hyperkähler manifolds, Journal of Differential geometry 55 (2000), 355-384.

[Don90] Simon K. Donaldson, Polynomial Invariants for Smooth FourManifolds, Topology 29 (1990), no. 3, 257-315.

[Fue35] R. Fueter, Die Funktionentheorie der Differentialgleichungen $\delta u=0$ und $\delta \delta u=0$ mit vier reellen Variablen, Commentarii mathematici Helvetici 7 (1934/35), 307-330.

[GBL88] K. Galicki and Jr. B.H. Lawson, Quaternionic reduction and Quaternionic Orbifolds, Math. Ann. 282 (1988), no. 1, 1-21.

[GS78] G.W.Gibbons and S.W.Hawking, Gravitational multi-instantons, Physics Letter 78B (1978), no. 4.

[Hay08] Andriy Haydys, Nonlinear Dirac operator and quaternionic analysis, Communications in Mathematical Physics 281 (2008), no. 1, 251-261.

[Hit74] Nigel Hitchin, Harmonic Spinors, Advances in Mathematics 14 (1974), $1-55$.

[Hit87]_, The self-duality equations on a riemann surface, Proc. London Math. Soc. 55 (1987), no. 1, 59-126.

[Hit92] _ Hyperkähler manifolds, Séminaire Bourbaki 34 (1991-1992), no. exp. no $748,137-166$.

[HKLR87] Hitchin, Karlhede, Lindström, and Roček., Hyperkähler metric and Supersymmetry, Communications in Mathematical Physics 108 (1987), no. $4,535-589$.

[Jac51] N. Jacobson, Completely reducible Lie algebras of linear transformations, Proceedings of American Mathematical Society 2 (1951), 105113.

[Kok94] Gerasim Kokarev, Curvature and bubble convergence of harmonic maps, arXiv:1005.3783 (1994). 
[Kro90] P. B. Kronheimer, A hyper-Kählerian structure on coadjoint orbits of a Semisimple complex group, Journal of the London Mathematical Society s2-42 (1990), no. 2, 193-208.

[Lic70] Lichnerowicz, Applications harmoniques et variétés kählériennes , Symp. Math. III, Bologna (1970), 341-402.

[Lin99] Fang-Hua Lin, Gradient estimates and blow-up analysis for stationary harmonic maps, Annals of Mathematics 149 (1999), no. 3, 785-829.

[LM89] Blaine Lawson and Marie Michelsohn, Spin Geometry, 38, Princeton University Press, 1989.

[LT98] Jiayu Li and Gang Tian, A Blow-Up Formula for Stationary Harmonic Maps, International Mathematics Research Notices 1998 (1998), no. $14,735-755$.

[Mar99] Matilda Marcolli, Seiberg-Witten Gauge Theory, TRIM, no. 17, Hindustan Book Agency, 1999.

[Mor60] V.V. Morozov, A theorem on the nilpotent element in a semi-simple Lie algebra, Uspekhi Mat. Nauk 15 (1960), no. 6(96), 137-139.

[Mor96] John W. Morgan, The Seiberg-Witten Equations and Applications to the Topology of Smooth Four-Manifolds, Mathematical Notes, vol. 44, Princeton University Press, 1996.

[Nic] Liviu I. Nicolaescu, Notes on Seiberg-Witten Theory, Graduate Studies in Mathematics, vol. 28, American Mathematical Society.

[Nic96]_ Lectures on the Geometry of Manifolds, World Scientific, 1996.

[Oba58] M. Obata, Hermitian manifolds with Quaternion structure, Tohoku Math. Journal 10 (1958), no. 1, 11-18.

[Pid04] V. Ya. Pidstrygach, HyperKähler manifolds and Seiberg-Witten equations, Proceedings of Steklov Institute of Mathematics (2004), 249-262.

[Pid13]_, Personal Communication. 
[Sal89] Simon Salamon, Riemannian geometry and holonomy groups, Pitman Research notes in Mathematics, vol. 201, Longman Scientific \& Technical, 1989.

[Sch84] Richard Schoen, Analytic aspects of the harmonic map problem, Seminar on Nonlinear Partial Differential Equations, Math. Sci. Res. Inst. Publ., no. 2, Springer-Verlag, New-York, 1984.

[Sch10] Henrik Schumacher, Generalized Seiberg-Witten equations: Swann bundles and $L^{\infty}$-estimates, Master's thesis, Mathematisches Institut, Georg-August-Universität, Göttingen, http://www.uni-math.gwdg. de/preprint/mg.2010.02.pdf, 2010.

[Sim83] Leon Simon, Lectures on Geometric Measure Theory, Proc. Centre Math. Appl. Austral. Nat. Univ., vol. 3, Australian National Univ. Press, Canberra, 1983.

[SU82] Richard Schoen and Karen Uhlenbeck, A Regularity Theory for Harmonic Maps, Journal of Differential Geometry 17 (1982), 307-335.

[Swa91] Andrew Swann, HyperKähler and Quaternionic Kähler geometry, Math. Ann. 3 (1991), 421-450.

[Tau99] C. H. Taubes, Nonlinear Generalizations of a 3-manifold's Dirac operator, Trends in mathematical physics (Knoxville, TN, 1998), AMS/IP Stud. Adv. Math., vol. 13, Amer. Math. Soc., Providence, RI, 1999, pp. $475-486$.

[Wit94] Edward Witten, Monopoles and Four-Manifolds, arXiv:hepth/9411102 (1994).

[Wol65] J. A. Wolf, Complex Homogeneous Contact manifolds and Quaternionic symmetric spaces, Journal of Mathematics and Mechanics 14 (1965), no. $6,1033-1047$. 